Pacific

Journal of

Mathematics

SELF-SIMILAR SOLUTIONS OF THE $\boldsymbol{p}$-LAPLACE HEAT EQUATION: THE FAST DIFFUSION CASE

MARIE FrançOise Bidaut-VÉron 


\title{
SELF-SIMILAR SOLUTIONS OF THE $p$-LAPLACE HEAT EQUATION: THE FAST DIFFUSION CASE
}

\author{
MARIE FranÇOISE BIDAUT-VÉRON
}

\begin{abstract}
We study the self-similar solutions of the equation $u_{t}-\operatorname{div}\left(|\nabla u|^{p-2} \nabla u\right)=0$ in $\mathbb{R}^{N}$, where $N \geq 1, p \in(1,2)$. We provide a complete description of the signed solutions of the form $u(x, t)=( \pm t)^{-\alpha / \beta} w\left(( \pm t)^{-1 / \beta}|x|\right)$, regular or singular at $x=0$, with $\alpha, \beta$ real, $\beta \neq 0$, and possibly not defined on all of $\mathbb{R}^{N} \times(\mathbf{0}, \pm \infty)$.
\end{abstract}

\section{Introduction and main results}

In this article we study the existence of self-similar solutions of the degenerate parabolic equation involving the $p$-Laplace operator in $\mathbb{R}^{N}, N \geq 1$,

$$
u_{t}-\operatorname{div}\left(|\nabla u|^{p-2} \nabla u\right)=0,
$$

with $1<p<2$. In the sequel we set

$$
\delta=\frac{p}{2-p}
$$

so $\delta>1$. Two critical values $P_{1}, P_{2}$ are involved in the problem

$$
P_{1}=\frac{2 N}{N+1}, \quad P_{2}=\frac{2 N}{N+2}
$$

see [DiBenedetto and Herrero 1990], for example. They are connected with $\delta$ through the relations

$$
p>P_{1} \Longleftrightarrow \delta>N, \quad p>P_{2} \Longleftrightarrow \delta>\frac{N}{2} .
$$

If $u(x, t)$ is a solution and $\alpha, \beta \in \mathbb{R}$, then $u_{\lambda}(x, t)=\lambda^{\alpha} u\left(\lambda x, \lambda^{\beta} t\right)$ is a solution of $\left(E_{u}\right)$ if and only if

$$
\beta=p-(2-p) \alpha=(2-p)(\delta-\alpha)
$$

MSC2000: primary 35K65; secondary 34C 35 .

Keywords: degenerate parabolic equations, self-similar solutions. 
thus $\beta>0$ if and only if $\alpha<\delta$. For given $\alpha \in \mathbb{R}$ such that $\alpha \neq \delta$, the natural way to construct particular solutions is to search for self-similar solutions, radially symmetric in $x$, of the form

$$
u=u(x, t)=(\varepsilon \beta t)^{-\alpha / \beta} w(r), \quad r=(\varepsilon \beta t)^{-1 / \beta}|x|,
$$

where $\varepsilon= \pm 1$. By translation, for any real $T$, we obtain solutions defined for any $t>T$ when $\varepsilon \beta>0$, or $t<T$ when $\varepsilon \beta<0$. The hypersurfaces $\{r=$ constant $\}$ are of focusing type if $\beta>0$ and of spreading type if $\beta<0$. We are led to the equation

$\left(E_{w}\right)$

$$
\left(\left|w^{\prime}\right|^{p-2} w^{\prime}\right)^{\prime}+\frac{N-1}{r}\left|w^{\prime}\right|^{p-2} w^{\prime}+\varepsilon\left(r w^{\prime}+\alpha w\right)=0 \quad \text { in }(0, \infty) .
$$

If we look for solutions of $\left(E_{u}\right)$ under the form

$$
u=A e^{-\varepsilon \mu t} w(r), \quad r=M e^{-\varepsilon \mu t / \delta}|x|, \quad \mu>0,
$$

then $w$ solves $\left(E_{w}\right)$ provided $M=\delta / \alpha$ and $A=\left(\delta^{p} / \alpha^{p-1} \mu\right)^{1 /(2-p)}$, where $\alpha>0$ is arbitrary. This is another motivation for studying equation $\left(E_{w}\right)$ for any real $\alpha$.

In the huge literature on self-similar solutions of parabolic equations, many results deal with positive solutions $u$ defined and smooth on $\mathbb{R}^{N} \times(0, \infty)$. Equation $\left(E_{w}\right)$ was studied in [Qi and Wang 1999] when $\alpha>0, \varepsilon=1$. In our work we provide an exhaustive description of the self-similar solutions of equation $\left(E_{u}\right)$, possibly not defined on all of $(0, \infty)$, with constant or changing sign. In particular, for suitable values of $\alpha$, we prove the existence of solutions $w$ oscillating with respect to 0 as $r$ tends to 0 or $\infty$, or constant-sign solutions oscillating with respect to some nonzero constant. Our main tool is the reduction of the problem to an autonomous system with two variables and two parameters, $p$ and $\alpha$. We are led to a dynamical system, which we study by phase-plane techniques. When $p=\frac{3}{2}$, this system is nearly quadratic, and many devices from the theory of algebraic dynamical systems can be used. In the general case such structures do not exist; then we use energy functions associated to the system. The behavior of the solutions presents great diversity, according to the possible values of $p$ and $\alpha$.

In the sequel we set

$$
\eta=\frac{N-p}{p-1}
$$

thus $\eta>0$ if $N \geq 2$, and $\eta=-1$ if $N=1$. Observe the relation connecting $\eta, \delta$ and $N$ :

$$
\frac{\delta-N}{p-1}=\delta-\eta=\frac{N-\eta}{2-p} .
$$

Explicit solutions. Obviously if $w$ is a solution of $\left(E_{w}\right)$, so is $-w$. Many particular solutions are well-known. 
The infinite point source solution $\boldsymbol{U}_{\infty}$. The simplest positive solutions of equation $\left(E_{w}\right)$, which exist for any $\alpha$ such that $\varepsilon(\delta-N)(\delta-\alpha)>0$, are given by

$$
w(r)=\ell r^{-\delta},
$$

where

$$
\ell=\left(\varepsilon \delta^{p-1} \frac{\delta-N}{\delta-\alpha}\right)^{1 /(2-p)}>0 .
$$

They correspond to a unique solution $u$ of $\left(E_{u}\right)$ called $U_{\infty}$ in [Chasseigne and Vazquez 2002], singular at $x=0$, for any $t \neq 0$ :

$$
U_{\infty}(x, t)=\left(\frac{C t}{|x|^{p}}\right)^{1 /(2-p)}, \quad C=(2-p) \delta^{p-1}(\delta-N) .
$$

The case $\alpha=N$. Here the equation $\left(E_{w}\right)$ has a first integral

$$
w+\varepsilon r^{-1}\left|w^{\prime}\right|^{p-2} w^{\prime}=C r^{-N} .
$$

All the solutions corresponding to $C=0$ are given by

$$
\begin{aligned}
& w=w_{K, \varepsilon}(r)= \pm\left(\varepsilon \delta^{-1} r^{p^{\prime}}+K\right)^{-\delta / p^{\prime}} \\
& u= \pm u_{K, \varepsilon}(x, t)=\left(\varepsilon \beta_{N} t\right)^{-N / \beta_{N}}\left(\varepsilon \delta^{-1}\left(\varepsilon \beta_{N} t\right)^{-p^{\prime} / \beta_{N}}|x|^{p^{\prime}}+K\right)^{-(p-1) /(2-p)}, \\
& K \in \mathbb{R}
\end{aligned}
$$

with $\beta=\beta_{N}=(N+1)\left(p-P_{1}\right)$. For $p>P_{1}, \varepsilon=1, K>0$, the solutions are named after Barenblatt [1952]. For given $c>0$, the function $u_{K, 1}$, defined on $\mathbb{R}^{N} \times(0, \infty)$, is the unique solution of equation $\left(E_{u}\right)$ with initial data $u(0)=c \delta_{0}$, $\delta_{0}$ being the Dirac mass at 0 and $K$ begin determined by $\int_{\mathbb{R}^{N}} u_{K}(x, t) d t=c$; see for example [Zhao 1993]. Moreover the functions $u_{K, 1}$, with $K>0$, are the only nonnegative solutions defined on $\mathbb{R}^{N} \times(0, \infty)$, such that $u(x, 0)=0$ for any $x \neq 0$; see [Kamin and Vázquez 1992]. In the case $K=0$, we find again the function $U_{\infty}$, and $U_{\infty}$ is the limit of the functions $u_{K, 1}$ as $K \rightarrow 0$, or equivalently $c \rightarrow \infty$.

The case $\alpha=\eta$. We exhibit a family of solutions of $\left(E_{w}\right)$ :

$$
w(r)=C r^{-\eta}, \quad u(t, x)=C|x|^{-\eta}=C|x|^{(p-N) /(p-1)}, \quad C \neq 0,
$$

Solutions $u$, independent of $t$, are the fundamental $p$-harmonic solutions of the equation when $p>P_{1}$.

The case $\alpha=-\boldsymbol{p}^{\prime}$. Equation $\left(E_{w}\right)$ admits solutions of the form

$$
\begin{aligned}
w(r) & = \pm K\left(N\left(K p^{\prime}\right)^{p-2}+\varepsilon r^{p^{\prime}}\right), \\
u(x, t) & = \pm K\left(N\left(K p^{\prime}\right)^{p-2} t+\varepsilon|x|^{p^{\prime}}\right), \quad K>0,
\end{aligned}
$$


and the functions $u$ are solutions of the form $\psi(t)+\Phi(|x|)$ with $\Phi$ nonconstant. They have constant sign when $\varepsilon=1$, and a changing sign when $\varepsilon=-1$.

The case $\boldsymbol{\alpha}=\mathbf{0}$. Here equation $\left(E_{w}\right)$ can be explicitly solved: either $w^{\prime} \equiv 0$ (hence $w \equiv a \in \mathbb{R}$, and $u$ is a constant solution of $\left.\left(E_{u}\right)\right)$, or there exists $K \in \mathbb{R}$ such that

$$
\left|w^{\prime}\right|=r^{(1-N) /(p-1)} \times \begin{cases}\left(K+\frac{\varepsilon}{\delta-N} r^{N-\eta}\right)^{-1 /(2-p)} & \text { if } \delta \neq N, \\ \left(\frac{2-p}{p-1}(K+\varepsilon \ln r)\right)^{-1 /(2-p)} & \text { if } \delta=N,\end{cases}
$$

which gives $w$ by integration, up to a constant, and then $u(x, t)=w\left(|x| /(\varepsilon p t)^{1 / p}\right)$.

The case $N=1$ and $\alpha=(p-1) /(2-p)>0$. Here again we obtain explicit solutions:

$w(r)= \pm\left(\varepsilon K\left(r-(K \alpha)^{p-1}\right)\right)^{-\alpha}, \quad u(x, t)= \pm\left(\varepsilon K\left(|x|-\varepsilon(K \alpha)^{p-1} t\right)\right)^{-\alpha}, \quad K>0$.

All the functions $w$ above are defined on intervals of the form $(R, 0), R \geq 0$ if $\varepsilon=1$, and $(0, S), S \leq \infty$ if $\varepsilon=-1$.

Note. When $\alpha=\delta$, equation $\left(E_{u}\right)$ is invariant under the transformation $u_{\lambda}(x, t)=$ $\lambda^{\alpha} u(\lambda x, t)$; searching solutions of the form $u(x, t)=|x|^{-\delta} \psi(t)$, we find again the function $U_{\infty}$.

Different kinds of singularities. Consider equation $\left(E_{w}\right)$. It is easy to get local existence and uniqueness near any point $r_{1}>0$; thus any solution $w$ is defined on a maximal interval $\left(R_{w}, S_{w}\right)$, with $0 \leq R_{w}<S_{w} \leq \infty$; and in fact $S_{w}=\infty$ when $\varepsilon=1$, and $R_{w}=0$ when $\varepsilon=-1$ (see Theorem 2.2). Returning to solution the $u$ of $\left(E_{u}\right)$ associated to $w$ by $(1-1)$, it is defined on a subset of $\mathbb{R}^{N} \backslash\{0\} \times(0, \pm \infty)$ :

$$
D_{w}=\left\{(x, t): x \in \mathbb{R}^{N}, \varepsilon \beta t>0,(\varepsilon \beta t)^{1 / \beta} R_{w}<|x|<(\varepsilon \beta t)^{1 / \beta} S_{w}\right\} .
$$

When $w$ is defined on $(0, \infty)$, then $u$ is defined on $\mathbb{R}^{N} \backslash\{0\} \times(0, \pm \infty)$.

Regular solutions. Among the solutions of $\left(E_{w}\right)$ defined near 0 , we also show the existence and uniqueness of solutions $w=w(., a) \in C^{2}\left(\left[0, S_{w}\right)\right)$ such that, for some $a \in \mathbb{R}$,

$$
w(0)=a, \quad w^{\prime}(0)=0 .
$$

These are called regular solutions. Obviously, they are defined on $[0, \infty)$ when $\varepsilon=1$. If $w$ is regular, then $D_{w}=\mathbb{R}^{N} \times(0, \pm \infty)$, and $u(., t) \in C^{1}\left(\mathbb{R}^{N}\right)$ for $t \neq 0$; we will say that $u$ is regular. This does not imply the regularity up to $t=0$ : indeed $u$ presents a singularity at time $t=0$ if and only if $0<\alpha<\delta$. In the sequel we shall not mention the trivial solution $w \equiv 0$, corresponding to $a=0$. 
Singular solutions. If $R_{w}=0$ and $w$ is not regular, $u$ presents a singularity at $x=0$ for $t \neq 0$, called a standing singularity. Following [Vazquez and Véron 1996; Chasseigne and Vazquez 2002], for such a solution, we say that $x=0$ is a weak singularity if $x \mapsto w(|x|) \in L_{\text {loc }}^{1}\left(\mathbb{R}^{N}\right)$, or equivalently if $u(., t) \in L_{\text {loc }}^{1}\left(\mathbb{R}^{N}\right)$ for $t \neq 0$; and a strong singularity if not. If $u$ has a strong/weak singularity, and $\lim _{t \rightarrow 0} u(t, x)=0$ for any $x \neq 0$, we call $u$ a strong/weak razor blade. If $u(., t) \in$ $L^{1}\left(\mathbb{R}^{N}\right)$ for $t \neq 0$, then $u$ is called integrable.

Solutions with a reduced domain. If $R_{w}>0$ or $S_{w}<\infty$, we say that $u$ and $w$ have a reduced domain. Then $D_{w}$ has a lateral boundary of the form $\Sigma_{w}=\{|x|=$ $C(\varepsilon \beta t)^{1 / \beta}$, of parabolic type if $\beta>0$ and of hyperbolic type if $\beta<0$, and $u$ has an explosion near $\Sigma_{w}$. In Proposition 2.15 we calculate the blow-up rate, which is of the order of $d(x, t)^{-(p-1) /(2-p)}$, where $d(x, t)$ is the distance to $\Sigma_{w}$.

Main results. We give a summary of our main results, expressed in terms of the function $u$, avoiding for simplicity particular cases (such as $N=1$, or $\alpha=\delta$, or $p=P_{1}$ ) and solutions with a reduced domain (although there exist many such). All cases omitted here and detailed statements in terms of $w$ can be found inside each section. An important critical value of $\alpha$ is given by

$$
\alpha^{*}=\delta+\frac{\delta(N-\delta)}{(p-1)(2 \delta-N)} ;
$$

it appears when $\varepsilon=1, p>P_{2}$, and then $\alpha^{*}>0$, or $\varepsilon=-1, p<P_{2}$, and then $\alpha^{*}<0$.

Note. To return from $w$ to $u$, consider any solution $w$ of $\left(E_{w}\right)$ defined on $(0, \infty)$, such that for some $\lambda \geq 0$ and $\mu \in \mathbb{R}, \lim _{r \rightarrow 0} r^{\lambda} w=c \neq 0$ and $\lim _{r \rightarrow 0} r^{\mu} w=c^{\prime} \neq 0$. Then:

(i) For fixed $t, u$ has a singularity in $|x|^{-\lambda}$ near $x=0$, and a behavior in $|x|^{-\mu}$ for large $|x|$. Thus $x=0$ is a weak singularity if and only if $\lambda<N$, and $u$ is integrable if and only if $\lambda<N<\mu$.

(ii) For fixed $x \neq 0$, the behavior of $u$ near $t=0$, depends on the sign of $\beta$ :

$$
\begin{array}{ll}
\lim _{t \rightarrow 0}|x|^{\mu}|t|^{(\alpha-\mu) / \beta} u(x, t)=C \neq 0 & \text { if } \alpha<\delta, \\
\lim _{t \rightarrow 0}|x|^{\lambda}|t|^{(\alpha-\lambda) / \beta} u(x, t)=C \neq 0 & \text { if } \delta<\alpha .
\end{array}
$$

Solutions defined for $\boldsymbol{t}>\mathbf{0}$. Here we look for solutions $u$ of $\left(E_{u}\right)$ on $\mathbb{R}^{N} \backslash\{0\} \times$ $(0, \infty)$ of the form (1-1). That means $\varepsilon \beta>0$, or equivalently $\varepsilon=1$ and $\alpha<\delta$ (see Section 3) or $\varepsilon=-1, \delta<\alpha$ (see Section 4). We begin with the case $\varepsilon=1$, and examine the dependence on the sign of $p-P_{1}$. For proofs, see Theorems 3.2, 3.4 and 3.5. 
Theorem 1.1. Assume $\varepsilon=1,-\infty<\alpha<\delta, p>P_{1}$, and $N \geq 2$. Then $U_{\infty}$ is a solution on $\mathbb{R}^{N} \backslash\{0\} \times(0, \infty)$ and a strong razor blade. There exist also positive solutions having a strong singularity in $|x|^{-\delta}$ and satisfying $\lim _{t \rightarrow 0}|x|^{\alpha} u=L>0$ $($ for $x \neq 0)$. For $\alpha \leq N$, any function $u(., t)$ has at most one zero at time $t$.

(1) For $\alpha<N$, all regular solutions on $\mathbb{R}^{N} \times(0, \infty)$ have constant sign, are not integrable, and they are solutions of $\left(E_{u}\right)$ with initial data $L|x|^{-\alpha} \in L_{\mathrm{loc}}^{1}\left(\mathbb{R}^{N}\right)$. There exist positive integrable razor blades having a singularity in $|x|^{-\eta}$. There exist also positive solutions having a weak regularity in $|x|^{-\eta}$ and satisfying $\lim _{t \rightarrow 0}|x|^{\alpha} u=L$; in particular if $\alpha=\eta$, then $u \equiv C|x|^{-\eta}$. There exist solutions with one zero and a weak or a strong singularity.

(2) For $\alpha=N$, all regular (Barenblatt) solutions have constant sign and are integrable. There exist solutions with one zero and a weak singularity.

(3) For $N<\alpha$, all regular solutions have at least one zero. If $\alpha<\alpha^{*}$, any solution has a finite number of zeros. If $N<\alpha^{*}$, there exists $\check{\alpha} \in\left(\alpha^{*}, \delta\right)$ such that if $\check{\alpha}<\alpha$, regular solutions are oscillating around 0 for large $|x|$, and $r^{\delta} w$ is asymptotically periodic in $\ln r$; and there exists precisely a solution $u$ such that $r^{\delta} w$ is periodic in $\ln r$.

Theorem 1.2. Assume $\varepsilon=1,-\infty<\alpha<\delta$, and $p<P_{1}$. Then all regular solutions on $\mathbb{R}^{N} \times(0, \infty)$ have constant sign, are not integrable, and are solutions of $\left(E_{u}\right)$ with initial data $L|x|^{-\alpha} \in L_{\text {loc }}^{1}\left(\mathbb{R}^{N}\right)$. There is no other solution on $\mathbb{R}^{N} \backslash\{0\} \times(0, \infty)$.

If $\alpha>0$, all the solutions $w$ tend to 0 at $\infty$, whereas if $\alpha<0$, some of the solutions are unbounded near $\infty$.

Next we come to the case $\varepsilon=-1$, which is treated in Theorems 4.1 and 4.2.

Theorem 1.3. Assume $\varepsilon=-1, \delta<\alpha, p>P_{1}$, and $N \geq 2$. There is no regular solution on $\mathbb{R}^{N} \times(0, \infty)$. Besides the function $U_{\infty}$, which is a strong razor blade, there exist positive integrable razor blades having a singularity in $|x|^{-\eta}$, and positive solutions having a strong singularity in $|x|^{-\alpha}$ and satisfying $\lim _{t \rightarrow 0}|x|^{\alpha} u=L$.

Theorem 1.4. Assume $\varepsilon=-1, \delta<\alpha, p<P_{1}(N \geq 2)$. There is no regular solution on $\mathbb{R}^{N} \times(0, \infty)$. There exists a positive solution on $\mathbb{R}^{N} \backslash\{0\} \times(0, \infty)$ with a singularity in $|x|^{-\alpha}$ (strong if and only if $N \leq \alpha$ ), and $\lim _{t \rightarrow 0}|x|^{\alpha} u=L$.

Note. Weak singularities can occur even if $p>P_{1}$. For example, the solutions $u(t, x)=C|x|^{-\eta}=C|x|^{(p-N) /(p-1)}(N \geq 2)$ given in (1-7) have a weak singularity. There even exist positive solutions $u$ with a standing singularity, and integrable; see Theorems 1.1 and 1.3. This is not contradictory with the regularizing effect $L_{\text {loc }}^{1}\left(\mathbb{R}^{N}\right) \rightarrow L_{\text {loc }}^{\infty}\left(\mathbb{R}^{N}\right)$, which concerns solutions in $(0, \infty) \times \mathbb{R}^{N}$. The functions constructed above are solutions in $(0, \infty) \times \mathbb{R}^{N} \backslash\{0\}$, and the singularity $x=0$ is not removable. 
Solutions defined for $\boldsymbol{t}<\mathbf{0}$. Next we consider the solutions defined for $t<0$, and more generally for $t<T$. They correspond to $\varepsilon=1, \delta<\alpha$ (Section 5), or $\varepsilon=-1, \alpha<\delta$ (Section 6). A main question in that case is the extinction problem: do there exist regular solutions $u$ vanishing identically on $\mathbb{R}$ at time $T$ ? Do there exist singular razor blades, vanishing on $\mathbb{R}^{N} \backslash\{0\}$ at time $T$ ? Are they integrable?

One of our most significant results is the existence of two critical values $\alpha_{\text {crit }}>0$ (when $P_{2}<p<P_{1}$ ) and $\alpha^{\text {crit }}<0$ (when $1<p<P_{2}$ ), for which the regular solutions $u_{\alpha_{\text {crit }}}$ are positive, integrable, and vanish identically at time 0 . Another new phenomena is the existence of positive solutions such that $C_{1} U_{\infty} \leq u \leq C_{2} U_{\infty}$ for some $C_{1}, C_{2}>0$, with a periodicity property, see Theorems 1.6 and 1.8 .

First assume $\varepsilon=1$. From Theorems 5.1 when $p>P_{1}$ and 5.4, 5.6, 5.7 when $p<P_{1}$, we deduce:

Theorem 1.5. Assume $\varepsilon=1, \delta<\alpha, p>P_{1}$, with $N \geq 2$. Any solution $u$ on $\mathbb{R}^{N} \backslash\{0\} \times(0,-\infty)$, in particular the regular ones, is oscillating around 0 for fixed $t<0$ and large $|x|$, and $r^{\delta} w$ is asymptotically periodic in $\ln r$. There exists a solution such that $r^{\delta} w$ is periodic in $\ln r$. There exist weak integrable razor blades, with a singularity in $|x|^{-\eta}$.

Theorem 1.6. Assume $\varepsilon=1, \delta<\alpha, p<P_{1}$. Then $U_{\infty}$ is a solution on $\mathbb{R}^{N} \backslash\{0\} \times$ $(0,-\infty)$, and a weak razor blade.

(1) If $p<P_{2}$, all regular solutions on $\mathbb{R}^{N} \times(0,-\infty)$ have constant sign, are not integrable, and vanish identically at $t=0$, with $\|u(., t)\|_{L^{\infty}\left(\mathbb{R}^{N}\right)} \leq C|t|^{\alpha /|\beta|}$. All the solutions have a finite number of zeros.

(2) For $\alpha<\eta$, regular solutions have constant sign, with the same behavior (given by (1-6) if $\alpha=N$ ). There exists a positive solution $u$, which is not integrable, with a singularity in $|x|^{-\alpha}$ (a strong one if and only if $\alpha \geq N$ ), and $\lim _{t \rightarrow 0}|x|^{\alpha} u=L$. If $\alpha=\eta$, then $u(t, x)=C|x|^{-\eta}$ is a solution with a strong singularity.

(3) If $p>P_{2}$, there exists a critical value $\alpha_{\text {crit }}$ such that $\eta<\alpha_{\text {crit }}<\alpha^{*}$ and the regular solutions $u_{\alpha_{\text {crit }}}$ have constant sign, are integrable, and vanish identically at $t=0$, with $\|u(., t)\|_{L^{\infty}\left(\mathbb{R}^{N}\right)} \leq C|t|^{\alpha /|\beta|}$.

(4) If $\alpha \in\left(\alpha_{\text {crit }}, \alpha^{*}\right)$, there exist positive solutions $u$ such that $r^{\delta} w$ is periodic in $\ln r ;$ thus

$$
C_{1} U_{\infty} \leq u \leq C_{2} U_{\infty} \quad \text { for some } C_{1}, C_{2}>0 .
$$

There exist positive solutions $u$, with the same bounds, such that $r^{\delta} w$ is asymptotically periodic near 0 . There exist positive integrable solutions $u$ such that $r^{\delta} w$ is asymptotically periodic near 0. 
(5) If $\alpha_{\text {crit }}<\alpha$, all regular solutions are oscillating around 0 for fixed $t<0$ and large $|x|$, and $r^{\delta} w$ is asymptotically periodic in $\ln r$. There exist solutions oscillating around 0, such that $r^{\delta} w$ is periodic. If $\alpha^{*}<\alpha$, there exist positive integrable razor blades, with a singularity in $|x|^{-\delta}$.

Finally suppose $\varepsilon=-1$. From Theorems $6.1,6.2$ when $p>P_{1}$ and 6.4, 6.6, $6.8,6.9$ when $p<P_{1}$, we obtain:

Theorem 1.7. Assume $\varepsilon=-1, \alpha<\delta$ and $p>P_{1}$, with $N \geq 2$. If $\alpha>0$, there exist positive solutions $u$ with a weak singularity in $|x|^{-\eta}$, integrable if and only if $\alpha>N$, and $\lim _{t \rightarrow 0}|x|^{\alpha} u=L$. If $\alpha<0$, any solution has at least a zero. If $-p^{\prime}<\alpha$, there is no regular solution on $\mathbb{R}^{N} \times(0,-\infty)$. If $\alpha=-p^{\prime}$, all regular solutions, given by (1-8), have one zero.

Theorem 1.8. Assume $\varepsilon=-1, \alpha<\delta$ and $p<P_{1}$. Then $U_{\infty}$ is a solution on $\mathbb{R}^{N} \backslash\{0\} \times(0,-\infty)$, and a weak razor blade.

(1) If $p>P_{2}$, all the solutions have a finite number of zeros. There exist positive integrable razor blades, with a singularity in $|x|^{-\delta}$.

(2) If $-p^{\prime}<\alpha$, there is no regular solution on $\mathbb{R}^{N} \times(0,-\infty)$. There exist positive integrable razor blades as above. If $\alpha>0$, there exist positive solutions $u$ with a weak singularity in $|x|^{-\delta}$, integrable if and only if $\alpha>N$, and $\lim _{t \rightarrow 0}|x|^{\alpha} u=L$. If $-p^{\prime}<\alpha<0$, there exist solutions with one zero and the same behavior. If $\alpha=-p^{\prime}$, all regular solutions, given by (1-8), have one zero.

(3) If $p<P_{2}$, there exists a critical value $\alpha^{\text {crit }}$ such that $\alpha^{*}<\alpha^{\text {crit }}<-p^{\prime}$ for which the regular solutions $u_{\alpha}$ crit have constant sign, are integrable, and vanish identically at $t=0$, with $\|u(., t)\|_{L^{\infty}\left(\mathbb{R}^{N}\right)} \leq C|t|^{\alpha /|\beta|}$.

(4) If $p<P_{2}$ and $\alpha \in\left(\alpha^{*}, \alpha^{\text {crit }}\right)$, there exist positive solutions $u$ such that $r^{\delta} w$ is periodic in $\ln r$, and thus

$$
C_{1} U_{\infty} \leq u \leq C_{2} U_{\infty} \quad \text { for some } C_{1}, C_{2}>0 .
$$

There exist positive solutions with a weak singularity in $|x|^{-\delta}$, with the same bounds, such that $r^{\delta} w$ is asymptotically periodic near $\infty$. The regular solutions have constant sign, are not integrable, vanish identically at $t=0$, and $r^{\delta} w$ is asymptotically periodic near $\infty$.

(5) If $p<P_{2}$ and $\alpha<\alpha^{\text {crit }}$, there exist solutions oscillating around 0 , such that $r^{\delta} w$ is periodic. There exist solutions oscillating around 0 , integrable, such that $r^{\delta} w$ is asymptotically periodic. If $\alpha \leq \alpha^{*}$, all regular solutions have constant sign, are not integrable, and vanish identically at $t=0$. 
Note. If $p<P_{1}$, recall that the Harnack inequality does not hold, as can be shown by the regular positive solutions constructed in Theorem 1.6, in particular those given by (1-6) when $\alpha=N$. The two kinds of regular, integrable, solutions constructed for the critical values $\alpha_{\text {crit }}>0$ and $\alpha^{\text {crit }}<0$ are of different types: the first, constructed for $p>P_{2}$, disappears in a spreading way, the second, for $p<P_{2}$, disappears in a focusing way.

The case $p>2$ will be treated in a second article [Bidaut-Véron 2006b], where we complete the results of [Gil and Vázquez 1997].

\section{General properties}

Different formulations of the problem. In the remainder of the article we can assume that $\boldsymbol{\alpha} \neq \mathbf{0}$, since the solutions are given explicitly by (1-9) when $\alpha=0$. Defining

$$
\begin{aligned}
J_{N}(r) & =r^{N}\left(w+\varepsilon r^{-1}\left|w^{\prime}\right|^{p-2} w^{\prime}\right), \\
J_{\alpha}(r) & =r^{\alpha-N} J_{N}(r),
\end{aligned}
$$

$\left(E_{w}\right)$ can be written in an equivalent way under the form

$$
\begin{aligned}
J_{N}^{\prime}(r) & =r^{N-1}(N-\alpha) w, \\
J_{\alpha}^{\prime}(r) & =-\varepsilon(N-\alpha) r^{\alpha-2}\left|w^{\prime}\right|^{p-2} w^{\prime} .
\end{aligned}
$$

If $\alpha=N$, then $J_{N}$ is constant, so we find again (1-5).

We shall often use the following logarithmic substitution; for given $d \in \mathbb{R}$, setting

$$
w(r)=r^{-d} y_{d}(\tau), \quad Y_{d}=-r^{(d+1)(p-1)}\left|w^{\prime}\right|^{p-2} w^{\prime}, \quad \tau=\ln r,
$$

we obtain the equivalent system

$$
\begin{aligned}
& y_{d}^{\prime}=d y_{d}-\left|Y_{d}\right|^{(2-p) /(p-1)} Y_{d}, \\
& Y_{d}^{\prime}=(p-1)(d-\eta) Y_{d}+\varepsilon e^{(p+(p-2) d) \tau}\left(\alpha y_{d}-\left|Y_{d}\right|^{(2-p) /(p-1)} Y_{d}\right) .
\end{aligned}
$$

And $y_{d}, Y_{d}$ satisfy the equations

$$
\begin{aligned}
& \text { (2-5) } \quad y_{d}^{\prime \prime}+(\eta-2 d) y_{d}^{\prime}- d(\eta-d) y_{d} \\
&+\frac{\varepsilon}{p-1} e^{((p-2) d+p) \tau}\left|d y_{d}-y_{d}^{\prime}\right|^{2-p}\left(y_{d}^{\prime}+(\alpha-d) y_{d}\right)=0, \\
&(2-6) \quad Y_{d}^{\prime \prime}+(p-1)\left(\eta-2 d-p^{\prime}\right) Y_{d}^{\prime}+\varepsilon e^{((p-2) d+p) \tau}\left|Y_{d}\right|^{\frac{2-p}{p-1}}\left(\frac{Y_{d}^{\prime}}{p-1}+(\alpha-d) Y_{d}\right) \\
&-(p-1)^{2}(\eta-d)\left(p^{\prime}+d\right) Y_{d}=0 .
\end{aligned}
$$


Reduction to an autonomous system. The substitution (2-3) with $d=\delta$ is the most helpful: setting

$$
y=y_{d}, \quad w(r)=r^{-\delta} y(\tau), \quad Y=-r^{(\delta+1)(p-1)}\left|w^{\prime}\right|^{p-2} w^{\prime}, \quad \tau=\ln r,
$$

we are led to the autonomous system that plays a key role in the sequel:

$$
\begin{aligned}
y^{\prime} & =\delta y-|Y|^{(2-p) /(p-1)} Y, \\
Y^{\prime} & =(\delta-N) Y+\varepsilon\left(\alpha y-|Y|^{(2-p) /(p-1)} Y\right) .
\end{aligned}
$$

Since $N-\delta p=\eta-2 \delta$ and $N-\delta=(p-1)(\eta-\delta)$, Equation (2-5) takes the form

$\left(E_{y}\right)(p-1) y^{\prime \prime}+(N-\delta p) y^{\prime}+\delta(\delta-N) y+\varepsilon\left|\delta y-y^{\prime}\right|^{2-p}\left(y^{\prime}+(\alpha-\delta) y\right)=0$,

while Equation (2-6) becomes

$$
\begin{aligned}
\left(E_{Y}\right) \quad Y^{\prime \prime}+(N-2 \delta) Y^{\prime}+\frac{\varepsilon}{p-1}|Y|^{(2-p) /(p-1)} Y^{\prime} \\
+\varepsilon(\alpha-\delta)|Y|^{(2-p) /(p-1)} Y+\delta(\delta-N) Y=0 .
\end{aligned}
$$

When $w$ has constant sign, we define two functions associated to $(y, Y)$ :

$$
\begin{gathered}
\zeta(\tau)=\frac{|Y|^{(2-p) /(p-1)} Y}{y}(\tau)=-\frac{r w^{\prime}(r)}{w(r)}, \\
\sigma(\tau)=\frac{Y}{y}(\tau)=-\frac{\left|w^{\prime}(r)\right|^{p-2} w^{\prime}(r)}{r w(r)} .
\end{gathered}
$$

They play an essential role in the asymptotic behavior: $\zeta$ describes the behavior of $w^{\prime} / w$ and $\sigma$ is the slope in the phase plane $(y, Y)$. They satisfy the equations

$$
\zeta^{\prime}=\zeta(\zeta-\eta)+\frac{\varepsilon}{p-1}|\zeta y|^{2-p}(\alpha-\zeta)=\zeta\left(\zeta-\eta+\frac{\varepsilon(\alpha-\zeta)}{(p-1) \sigma}\right)
$$

$$
\sigma^{\prime}=\varepsilon(\alpha-N)+\left(|\sigma y|^{\frac{2-p}{p-1}} \sigma-N\right)(\sigma-\varepsilon)=\varepsilon(\alpha-N)+(\zeta-N)(\sigma-\varepsilon) .
$$

Note. Since $(S)$ is autonomous, for any solution $w$ of $\left(E_{w}\right)$ of the problem, all the functions $w_{\xi}(r)=\xi^{\delta} w(\xi r), \xi>0$, are also solutions. From uniqueness, all regular solutions are completely described from one of them: $w(r, a)=a w\left(a^{1 / \delta} r, 1\right)$; thus they present the same behavior at infinity.

System $(S)$ will be studied by using phase plane techniques, which was not done in [Qi and Wang 1999], and gives our main results. The set of trajectories of system $(S)$ in the phase plane $(y, Y)$ is symmetric with respect to $(0,0)$. We define

$$
\mathcal{M}=\left\{(y, Y) \in \mathbb{R}^{2}:|Y|^{(2-p) /(p-1)} Y=\delta y\right\},
$$


which is the set of the extremal points of $y$. We denote the four quadrants by

$$
\mathscr{2}_{1}=(0, \infty) \times(0, \infty), \quad 2_{2}=(-\infty, 0) \times(0, \infty), \quad 2_{3}=-\mathscr{2}_{1}, \quad 2_{4}=-2_{2} .
$$

Remarks 2.1. (i) The field at any point $(\xi, 0)$ with $\xi>0$ satisfies $y^{\prime}=-\xi^{1 /(p-1)}<$ 0 , and so points toward $2_{2}$. The field at any point $(\varphi, 0)$ with $\varphi>0$ satisfies $Y^{\prime}=\varepsilon \alpha \varphi$, and so points toward $2_{1}$ if $\varepsilon \alpha>0$ and toward $2_{4}$ if $\varepsilon \alpha<0$.

(ii) The pair $(y, Y)$ defined by $(2-7)$ is related to $J_{N}$ by the identity

$$
J_{N}(r)=r^{N-\delta}(y(\tau)-\varepsilon Y(\tau)), \quad \tau=\ln r
$$

and the formulae (2-2) can be recovered from the relations

$$
\begin{aligned}
(y-\varepsilon Y)^{\prime} & =(\delta-\alpha) y+\varepsilon(N-\delta) Y=(\delta-\alpha)(y-\varepsilon Y)+\varepsilon(N-\alpha) Y \\
& =(\delta-N)(y-\varepsilon Y)+(N-\alpha) y .
\end{aligned}
$$

(iii) In the sequel the monotonicity of the functions $y_{d}, Y_{d}$, in particular $y, Y, \zeta$ and $\sigma$ plays an important role. At any extremal point $\tau$, these functions satisfy

$$
\begin{aligned}
y_{d}^{\prime \prime}(\tau) & =y_{d}(\tau)\left(d(\eta-d)-\frac{\varepsilon(\alpha-d)}{p-1} e^{((p-2) d+p) \tau}\left|d y_{d(\tau)}\right|^{2-p}\right), \\
Y_{d}^{\prime \prime}(\tau) & =Y_{d}(\tau)\left((p-1)^{2}(\eta-d)\left(p^{\prime}+d\right)\right. \\
& \left.-\varepsilon(\alpha-d) e^{((p-2) d+p) \tau}\left|Y_{d}(\tau)\right|^{(2-p) /(p-1)}\right),
\end{aligned}
$$

(2-16) $(p-1) y^{\prime \prime}(\tau)=\delta^{2-p} y(\tau)\left(\delta^{p-1}(N-\delta)-\varepsilon(\alpha-\delta)|y(\tau)|^{2-p}\right)$

$$
=-|Y(\tau)|^{(2-p) /(p-1)} Y^{\prime}(\tau),
$$

(2-18) $\quad(p-1) \zeta^{\prime \prime}(\tau)=\varepsilon(2-p)\left((\alpha-\zeta)|\zeta|^{2-p}|y|^{-p} y y^{\prime}\right)(\tau)$

$$
=\varepsilon(2-p)\left((\alpha-\zeta)(\delta-\zeta)|\zeta y|^{2-p}\right)(\tau),
$$

$$
\begin{aligned}
(p-1) \sigma^{\prime \prime}(\tau) & =(2-p)\left((\sigma-\varepsilon)|\sigma|^{(2-p) /(p-1)} Y|y|^{(4-3 p) /(p-1)} y^{\prime}\right)(\tau) \\
& =\zeta^{\prime}(\tau)(\sigma(\tau)-\varepsilon)
\end{aligned}
$$

Energy functions for the system $(S)$. There is a classical energy function associated to equation $\left(E_{w}\right)$ :

$$
E(r)=\frac{1}{p^{\prime}}\left|w^{\prime}\right|^{p}+\varepsilon \frac{\alpha}{2} w^{2},
$$

which is nonincreasing when $\varepsilon=1$, since $E^{\prime}(r)=-(N-1) r^{-1}\left|w^{\prime}\right|^{p}-\varepsilon r w^{\prime 2}$. This is not sufficient in our study: we need energy functions adapted to $y$ and $Y$. 
Using the ideas of [Bidaut-Véron 1989], we construct two of them by using the Anderson and Leighton formula [1968].

We find a first function $W$ given by

$$
\begin{gathered}
W(\tau)=\mathscr{W}(y(\tau), Y(\tau)), \quad \text { where } \\
W(y, Y)=\varepsilon\left(\frac{(2 \delta-N) \delta^{p-1}}{p}|y|^{p}+\frac{|Y|^{p^{\prime}}}{p^{\prime}}-\delta y Y\right)+\frac{\alpha-\delta}{2} y^{2} .
\end{gathered}
$$

It satisfies

$$
\begin{aligned}
W^{\prime}(\tau)=\varepsilon(2 \delta-N)\left(\delta y-|Y|^{(2-p) /(p-1)} Y\right) & \left(|\delta y|^{p-2} \delta y-Y\right)-\left(\delta y-|Y|^{(2-p) /(p-1)} Y\right)^{2} \\
=\left(\delta y-|Y|^{(2-p) /(p-1)} Y\right)\left(|\delta y|^{p-2}\right. & \delta y-Y) \\
& \times\left(\varepsilon(2 \delta-N)-\frac{\delta y-|Y|^{(2-p) /(p-1)} Y}{|\delta y|^{p-2} \delta y-Y}\right) .
\end{aligned}
$$

When $\varepsilon(2 \delta-N) \leq 0$, then $W$ is nonincreasing. When $\varepsilon(2 \delta-N)>0$, we consider the curve

$$
\mathscr{L}=\left\{(y, Y) \in \mathbb{R}^{2}: H(y, Y)=\varepsilon(2 \delta-N)\right\},
$$

where

$$
H(y, Y):=\frac{\delta y-|Y|^{(2-p) /(p-1)} Y}{|\delta y|^{p-2} \delta y-Y}
$$

and by convention this quotient takes the value $|\delta y|^{2-p} /(p-1)$ if $|\delta y|^{p-2} \delta y=Y$. $\mathscr{L}$ is a closed curve surrounding $(0,0)$, symmetric with respect to $(0,0)$. Let $\mathscr{Y}_{\mathscr{L}}$ be the domain with boundary $\mathscr{L}$ and containing $(0,0)$ :

$$
\mathscr{S}_{\mathscr{L}}=\left\{(y, Y) \in \mathbb{R}^{2}: H(y, Y) \leq \varepsilon(2 \delta-N)\right\} .
$$

Then $W^{\prime}(\tau) \geq 0$ if $(y(\tau), Y(\tau)) \in \mathscr{Y}_{\mathscr{L}}$ and $W^{\prime}(\tau) \leq 0$ if $(y(\tau), Y(\tau)) \notin \mathscr{Y}_{\mathscr{L}}$. Observe that $\mathscr{S}_{\mathscr{L}}$ is bounded: indeed, for any $(y, Y) \in \mathbb{R}^{2}$,

$$
H(y, Y) \geq \frac{1}{2}\left((\delta y)^{2-p}+|Y|^{(2-p) /(p-1)}\right) .
$$

Also $\mathscr{S}_{\mathscr{L}}$ is connected; more precisely, for any $(y, Y) \in \mathscr{Y}_{\mathscr{L}}$ and any $\theta \in[0,1]$, we have $\left(\theta y, \theta^{p-1} Y\right) \in \mathscr{S}_{\mathscr{L}}$.

A second function, denoted by $V$, is also given by Anderson formula, or by multiplication by $Y^{\prime}$ in $\left(E_{Y}\right)$ : let

$$
\begin{gathered}
V(\tau)=\mathscr{V}\left(Y(\tau), Y^{\prime}(\tau)\right), \quad \text { where } \\
\mathscr{V}(Y, Z)=\frac{\varepsilon}{2}\left(\delta(\delta-N) Y^{2}+Y^{\prime 2}\right)+\frac{\alpha-\delta}{p^{\prime}}|Y|^{p^{\prime}} ;
\end{gathered}
$$

then

$$
V^{\prime}(\tau)=\left(\varepsilon(2 \delta-N)-\frac{1}{p-1}|Y|^{(2-p) /(p-1)}\right) Y^{\prime 2}
$$


When $\varepsilon(2 \delta-N)$ is not positive, $V$ is nonincreasing. When it is positive, we have $V^{\prime}(\tau) \geq 0$ whenever $|Y(\tau)| \leq D$, where

$$
D=(\varepsilon(2 \delta-N)(p-1))^{(p-1) /(2-p)} .
$$

The function $W$ gives more information on the system, because $\mathscr{S}_{\mathscr{L}}$ is bounded, whereas the set of zeros of $V^{\prime}$ is unbounded.

Stationary points of system $(S)$. If $\alpha=\delta=N$, system $(S)$ has infinitely many stationary points, given by $\pm\left(k,(\delta k)^{p-1}\right), k \geq 0$. Otherwise, if $\varepsilon(\delta-N)(\delta-\alpha) \leq 0$, the system has a unique stationary point $(0,0)$. If $\varepsilon(\delta-N)(\delta-\alpha)>0$, it admits the three stationary points

$$
(0,0), \quad M_{\ell}=\left(\ell,(\delta \ell)^{p-1}\right) \in \mathscr{2}_{1}, \quad M_{\ell}^{\prime}=-M_{\ell} \in \mathscr{2}_{3},
$$

where $\ell$ is defined in (1-4). In that case, we find again that $w \equiv \ell r^{-\delta}$ is a particular solution of equation $\left(E_{w}\right)$.

Local behavior at $(\mathbf{0 , 0})$. The linearized problem at $(0,0)$ is given by

$$
y^{\prime}=\delta y, \quad Y^{\prime}=(\delta-N) Y+\varepsilon \alpha y,
$$

and has eigenvalues $\mu_{1}=\delta-N$ and $\mu_{2}=\delta$. Thus $(0,0)$ is a saddle point when $\delta<N$ and a source when $N<\delta$. One can choose a basis of eigenvectors $v_{1}=(0,-1)$ and $v_{2}=(N, \varepsilon \alpha)$.

Local behavior at $M_{\ell}$. Setting

$$
y=\ell+\bar{y}, \quad Y=(\delta \ell)^{p-1}+\bar{Y},
$$

system $(S)$ is equivalent in $2_{1}$ to

$$
\bar{y}^{\prime}=\delta \bar{y}-\varepsilon v(\alpha) \bar{Y}-\Psi(\bar{Y}), \quad \bar{Y}^{\prime}=\varepsilon \alpha \bar{y}+(\delta-N-v(\alpha)) \bar{Y}-\varepsilon \Psi(\bar{Y}),
$$

where

$$
\begin{aligned}
v(\alpha) & =\frac{\delta(N-\delta)}{(p-1)(\alpha-\delta)} \\
\Psi(\vartheta) & =\left((\delta \ell)^{p-1}+\vartheta\right)^{1 /(p-1)}-\delta \ell-\frac{(\delta \ell)^{2-p}}{p-1} \vartheta
\end{aligned}
$$

with $\vartheta>-(\delta \ell)^{p-1}$. The linearized problem is given by

$$
\bar{y}^{\prime}=\delta \bar{y}-\varepsilon v(\alpha) \bar{Y}, \quad \bar{Y}^{\prime}=\varepsilon \alpha \bar{y}+(\delta-N-v(\alpha)) \bar{Y} .
$$

Its eigenvalues $\lambda_{1} \leq \lambda_{2}$ are the solutions of the equation

$$
\lambda^{2}-(2 \delta-N-v(\alpha)) \lambda+p^{\prime}(N-\delta)=0 .
$$


The discriminant $\Delta$ of this equation is

$$
\Delta=(2 \delta-N-v(\alpha))^{2}-4 p^{\prime}(N-\delta)=(N+v(\alpha))^{2}-4 v(\alpha) \alpha .
$$

The critical value $\alpha^{*}$ of $\alpha$, given in (1-11), arises when $\varepsilon(\delta-N / 2)>0$ :

$$
\alpha=\alpha^{*} \Longleftrightarrow \lambda_{1}+\lambda_{2}=0 .
$$

When $\delta<N$ and $\varepsilon=1$, then $\delta<\alpha$ and $M_{\ell}$ is a sink when $\delta \leq N / 2$ or $\delta>N / 2$ and $\alpha<\alpha^{*}$, and a source when $\delta>N / 2$ and $\alpha>\alpha^{*}$. When $\delta<N$, and $\varepsilon=-1$, then $\alpha<\delta$ and $M_{\ell}$ is a source when $\delta \geq N / 2$ or $\delta<N / 2$ and $\alpha>\alpha^{*}$, and a sink when $\delta<N / 2$ and $\alpha<\alpha^{*}$. When $N<\delta$, then $M_{\ell}$ is always a saddle point, but, as we will see later, the value $\alpha^{*}$ also plays a role.

More specifically, the sign of $\alpha^{*}$ and its position with respect to $N$ or $\eta$ play a role. By computation,

$$
\begin{aligned}
\alpha^{*} & =\frac{p^{\prime}\left(\delta^{2}-3 \delta+2 N\right)}{2(2 \delta-N)}=\eta+\frac{(\delta-N)^{2}}{(p-1)(2 \delta-N)} \\
& =N+\frac{(\delta-N)\left(\delta^{2}-(N+3) \delta+N\right)}{(2 \delta-N)(\delta-1)} .
\end{aligned}
$$

Thus, if $\varepsilon=1$, then $\alpha^{*}>\eta>0$ if $N \geq 2$; if $N=1, \alpha^{*}>0$ if $p>\frac{4}{3}$. If $\varepsilon=-1$, then $\alpha^{*}<-p^{\prime}<0$.

Otherwise, when $\Delta>0$ a basis of eigenvectors $u_{1}=\left(-\varepsilon v(\alpha), \lambda_{1}-\delta\right), u_{2}=$ $\left(\varepsilon \nu(\alpha), \delta-\lambda_{2}\right)$ can be chosen. If $\Delta \geq 0$, then $\delta$ is exterior to the roots if $\varepsilon \alpha>0$, and $\lambda_{1}<\delta<\lambda_{2}$ if $\varepsilon \alpha<0$.

\section{Existence of solutions of equation $\left(E_{w}\right)$.}

Theorem 2.2. (i) Take $r_{1}>0\left(r_{1} \geq 0\right.$ if $\left.N=1\right)$ and let $a, a^{\prime}$ be reals. There exists a unique solution $w$ of equation $\left(E_{w}\right)$ in a neighborhood $\mathscr{V}$ of $r_{1}$, such that $w \in C^{2}(\mathscr{V})$ and $w\left(r_{1}\right)=a, w^{\prime}\left(r_{1}\right)=a^{\prime}$. It has a unique extension to a maximal interval of the form

$$
\begin{aligned}
& \left(R_{w}, \infty\right) \text { with } 0 \leq R_{w} \quad \text { if } \varepsilon=1 \text {, } \\
& \left(0, S_{w}\right) \quad \text { with } S_{w} \leq \infty \quad \text { if } \varepsilon=-1 \text {. }
\end{aligned}
$$

If $0<R_{w}$ or $S_{w}<\infty$, as the case may be, $w$ is monotone near $R_{w}$ or $S_{w}$ with an infinite limit.

(ii) For any $a \in \mathbb{R}$, there exists a unique regular solution $w$ of $\left(E_{w}\right)$ satisfying (1-10), and

$$
\lim _{r \rightarrow 0}\left|w^{\prime}\right|^{p-2} w^{\prime} / r w=-\varepsilon \alpha / N
$$


(iii) If $N \geq 2$, any solution defined near 0 and bounded is regular. If $N=1$, it satisfies $\lim _{r \rightarrow 0} w^{\prime}=b \in \mathbb{R}$, and $\lim _{r \rightarrow 0} w=a \in \mathbb{R}$.

Proof. (i) Local existence and uniqueness near $r_{1}>0$ follow directly from Cauchy's theorem applied to equation $\left(E_{w}\right)$ or to system $(S)$, since the map $\xi \mapsto f_{p}(\xi)=$ $|\xi|^{(2-p) /(p-1)} \xi$ is of class $C^{1}$. If $N=1$, we can take $r_{1}=0$, obtain a local solution in a neighborhood of 0 in $\mathbb{R}$ and reduce it to $[0, \infty)$.

Any local solution around $r_{1}$ has a unique extension to a maximal interval $\left(R_{w}, S_{w}\right)$. Suppose that $0<R_{w}$ (or $S_{w}<\infty$ ) and that $w$ is oscillating around 0 near $R_{w}$ (or $S_{w}$ ). Making the substitution (2-3), with $d \neq 0$, if $\tau$ is a maximal point of $\left|y_{d}\right|$, we see that (2-14) holds. If we take $d$ such that $\varepsilon(d-\alpha)>0$, the sequence $\left(y_{d}(\tau)\right)$ stays bounded, since the exponential has a positive limit; for that reason $y_{d}$ stays bounded, $w$ is bounded near $R_{w}$ (or $S_{w}$ ) and then also $J_{N}^{\prime}$, $J_{N}$ and $w^{\prime}$, which is contradictory. Thus $w$ keeps a constant sign, for example $w>0$, near $R_{w}$ (or $S_{w}$ ). At each extremal point $r$ such that $w(r)>0$, we find $\left(\left|w^{\prime}\right|^{p-2} w^{\prime}\right)^{\prime}(r)=-\varepsilon \alpha w(r)$; thus $r$ is unique since $\alpha \neq 0$. Thus $w$ is strictly monotone near $R_{w}$ (or $S_{w}$ ), and $w$ and $\left|w^{\prime}\right|$ tend to $\infty$.

First suppose $\varepsilon=1$. We show that $S_{w}=\infty$. This is easy when $\alpha>0$ : since $E$ is nondecreasing, $w$ and $w^{\prime}$ are bounded for $r>r_{1}$. Assume $\alpha<0$ and $S_{w}<\infty$. Then for example $w$ is positive near $S_{w}$, nondecreasing, and $\lim _{r \rightarrow S_{w}} w=\infty$. Then $J_{\alpha}$ is nonincreasing and nonnegative near $S_{w}$; hence again $w$ and $w^{\prime}$ are bounded, which is contradictory.

Next suppose $\varepsilon=-1$. If $R_{w}>0$, for example, $w$ is positive and nonincreasing and $\lim _{r \rightarrow R_{w}} w=\infty$. Then either $\alpha<N$ and $J_{N}$ is nonnegative and nondecreasing near $R_{w}$, and thus bounded, or $\alpha \geq N$ and $J_{\alpha}$ is nonnegative and nondecreasing near $R_{w}$, and still bounded. In either case we reach a contradiction, then $R_{w}=0$.

(ii) By symmetry we can suppose $a \geq 0$. Let $\rho>0$. By (2-1) and (2-2), any regular solution $w$ on $[0, \rho]$ satisfies

$$
\begin{aligned}
w(r) & =a-\varepsilon \int_{0}^{r} f_{p}(s T(w)) d s, \\
T(w)(r) & =w(r)+(\alpha-N) \int_{0}^{1} \theta^{N-1} w(r \theta) d \theta .
\end{aligned}
$$

Conversely, any function $w \in C^{0}([0, \rho])$ that solves (2-34) satisfies $w \in C^{1}((0, \rho])$ and $\left|w^{\prime}\right|^{p-2} w^{\prime}(r)=r T(w)$; hence $\left|w^{\prime}\right|^{p-2} w^{\prime} \in C^{1}((0, \rho])$ and $w$ satisfies $\left(E_{w}\right)$ in $(0, \rho]$. And $\lim _{r \rightarrow 0} r T(w)=0$, thus $w \in C^{1}([0, \rho])$ and $\left|w^{\prime}\right|^{p-2} w^{\prime} \in C^{1}([0, \rho])$. Then $w$ satisfies $\left(E_{w}\right)$ in $[0, \rho]$ and $w^{\prime}(0)=0$. From $\left(E_{w}\right)$, we have

$$
\lim _{r \rightarrow 0}\left|w^{\prime}\right|^{p-2} w^{\prime} / r w=-\varepsilon \alpha / N,
$$


and therefore $w-a=O\left(r^{p^{\prime}}\right)$ near 0 . We look for $w$ of the form $a+r^{p^{\prime}} \zeta(r)$, with

$$
\zeta \in \mathscr{B}_{\rho, M}=\left\{\zeta \in C^{0}([0, \rho]):\|\zeta\|_{C^{0}([0, \rho])}=\max _{r \in[0, \rho]}|\zeta(r)| \leq M\right\} .
$$

We are led to the problem $\zeta=\Theta(\zeta)$, where

$$
\begin{aligned}
\Theta(\zeta)(r) & =-\varepsilon \int_{0}^{1} \theta^{1 /(p-1)} f_{p}\left(T\left(a+(r \theta)^{p^{\prime}} \zeta(r \theta)\right)\right) d \theta \\
& =-\varepsilon \int_{0}^{1} \theta^{1 /(p-1)} f_{p}\left(\frac{\alpha a}{N}+T\left((r \theta)^{p^{\prime}} \zeta(r \theta)\right)\right) d \theta .
\end{aligned}
$$

Taking for example $M=(|\alpha| a)^{1 /(p-1)}$, it follows that $\Theta$ is a strict contraction from $\mathscr{B}_{\rho, M}$ into itself for $\rho$ small enough, hence existence and uniqueness hold in $[0, \rho]$. (iii) If $w$ is defined in $(0, \rho)$ and bounded, then $J_{N}^{\prime}$ is integrable. Set

$$
l=\lim _{r \rightarrow 0} J_{N}(r) .
$$

Then $\left|w^{\prime}\right|^{p-2} w^{\prime}=\varepsilon l r^{1-N}(1+o(1)$. If $N \geq 2$, this implies $l=0$; thus from above, $w$ is regular. If $N=1$, then $\lim _{r \rightarrow 0} w^{\prime}=b \in \mathbb{R}$, and $\lim _{r \rightarrow 0} w=a \in \mathbb{R}$.

Definition. Suppose $p>1$. Let $\mathscr{T}_{r}$ be the trajectory in the plane $(y, Y)$ (see $(2-7))$ starting from $(0,0)$ at $-\infty$, with slope $\varepsilon \alpha / N$ and $y>0$ near time $-\infty$. Its opposite $-\mathscr{T}_{r}$ is also a trajectory with the same properties (except that $y<0$ ). Both are called regular trajectories. In this situation we say that $y$ is regular. Observe that $\mathscr{T}_{r}$ starts in $\mathscr{2}_{1}$ if $\varepsilon \alpha>0$, and in $\mathscr{2}_{4}$ if $\varepsilon \alpha<0$.

Remark 2.3. Let $w$ be any solution of $\left(E_{w}\right)$ such that $w>0$ on some interval $I$.

(i) The function $w$ has at most one extremal point on $I$, since $\left(\left|w^{\prime}\right|^{p-2} w^{\prime}\right)^{\prime}=$ $-\varepsilon \alpha w$, and this point is a maximum if $\varepsilon \alpha>0$ and a minimum if $\varepsilon \alpha<0$.

(ii) From (2-33), if $w$ is regular and $w>0$ in $\left(0, r_{1}\right), r_{1} \leq \infty$, then $w^{\prime}<0$ in $\left(0, r_{1}\right)$ when $\varepsilon \alpha>0$; thus $\mathscr{T}_{r}$ is in $\mathscr{2}_{1}$. And $w^{\prime}>0$ in $\left(0, r_{1}\right)$ when $\varepsilon \alpha<0$; hence $\mathscr{T}_{r}$ is in $\mathscr{2}_{3}$ in $\left(-\infty, \ln r_{1}\right)$.

Remark 2.4. In the case $\delta \neq N$, we can give a shorter proof of Theorem 2.2(ii). Indeed, $(0,0)$ is either a source or a saddle point. Thus there exists precisely one trajectory starting from $(0,0)$ at $-\infty$, with $y>0$, with slope $\varepsilon \alpha / N$. The corresponding solutions are regular: the slope $\sigma$ defined in (2-8) satisfies $\lim _{\tau \rightarrow-\infty} \sigma=\varepsilon \alpha / N$. Thus $\lim _{r \rightarrow 0}\left|w^{\prime}\right|^{p-2} w^{\prime} / r w=-\varepsilon \alpha / N$, implying that $w^{(2-p) /(p-1)}$ has a limit $a>0$. Since $\lim _{r \rightarrow 0} w^{\prime}=0$, this function $w$ satisfies (1-10), and any $a$ is obtained by scaling.

Notation. For any point $P_{0}=\left(y_{0}, Y_{0}\right) \in \mathbb{R}^{2} \backslash\{(0,0)\}$, the unique trajectory in the phase plane $(y, Y)$ going through $P_{0}$ is denoted by $\mathscr{T}_{\left[P_{0}\right]}$. Notice that $\mathscr{T}_{\left[-P_{0}\right]}=$ $-\mathscr{T}_{\left[P_{0}\right]}$, from the symmetry of system $(S)$. 


\section{First sign properties.}

Proposition 2.5. Let $w \not \equiv 0$ be any solution of $\left(E_{w}\right)$.

(i) If $\varepsilon=1$ and $\alpha \leq \max (N, \eta)$, then $w$ has at most one zero, and no zero if $w$ is regular.

(ii) If $\varepsilon=1$ and $N<\min (\delta, \alpha)$ and $w$ is regular, then $w$ has at least one zero.

(iii) If $\varepsilon=-1$ and $\alpha \geq \min (0, \eta)$, then $w$ has at most one zero. If $\alpha>0$ and $w$ is regular, then it has no zero.

(iv) If $\varepsilon=-1$ and $-p^{\prime} \leq \alpha<\min (0, \eta)$, then $w^{\prime}$ has at most one zero; consequently $w$ has at most two zeros, and at most one if $w$ is regular.

Proof. (i) Let $\varepsilon=1$. Take two consecutive zeros $\rho_{0}<\rho_{1}$ of $w$, with $w>0$ on $\left(\rho_{0}, \rho_{1}\right)$, so $w^{\prime}\left(\rho_{1}\right)<0<w^{\prime}\left(\rho_{0}\right)$. If $\alpha \leq N$, we find, using the function $J_{N}$ of $(2-1)$, $J_{N}\left(\rho_{1}\right)-J_{N}\left(\rho_{0}\right)=-\rho_{1}^{N-1}\left|w^{\prime}\left(\rho_{1}\right)\right|^{p-2}-\rho_{0}^{N-1} w^{\prime}\left(\rho_{0}\right)^{p-1}=(N-\alpha) \int_{\rho_{0}}^{\rho_{1}} s^{N-1} w d s$, which is contradictory; thus $w$ has at most one zero. If $w$ is regular with $w(0)>0$ and $\rho_{1}$ is a first zero, then

$$
J_{N}\left(\rho_{1}\right)=-\rho_{1}^{N-1}\left|w^{\prime}\left(\rho_{1}\right)\right|^{p-1}=(N-\alpha) \int_{0}^{\rho_{1}} s^{N-1} w d s \geq 0,
$$

again a contradiction. Next suppose $0<\alpha \leq \eta$ and use the substitution (2-3), with $d=\alpha$. Then $y_{\alpha}$ has at most one zero: indeed, if $y_{\alpha}$ has a maximal point $\tau$ where it is positive, and is not constant, then from (2-14),

$$
y_{\alpha}^{\prime \prime}(\tau)=\alpha(\eta-\alpha) y_{d}(\tau) ;
$$

hence $y_{\alpha}^{\prime \prime}(\tau)<0$, which is impossible. In the same way the regular solution satisfies $\lim _{\tau \rightarrow-\infty} y_{\alpha}=0$ since $\alpha>0$, and $y_{\alpha}$ has no maximal point; thus $y_{\alpha}$ is positive and increasing.

(ii) Let $\varepsilon=1$ and $w>0$ on $[0, \infty)$. If $N<\alpha$, then $J_{N}(r)=(N-\alpha) \int_{0}^{r} s^{N-1} w d s<0$. The function $r \mapsto \delta r^{p^{\prime}}-w^{(p-2) /(p-1)}$ is nonincreasing; hence $w=O\left(r^{-\delta}\right)$ at $\infty$, so $y$ is bounded at $\infty$. For any $r \geq 1$, one gets $J_{N}(r) \leq J_{N}(1)<0$, hence $y(\tau)+\left|J_{N}(1)\right| e^{(\delta-N) \tau} \leq Y(\tau)$ for any $\tau \geq 0$, from (2-12). Then $\lim _{\tau \rightarrow \infty} Y=\infty$, implying by $(S)$ that $\lim _{\tau \rightarrow \infty} y^{\prime}=-\infty$, which is impossible.

(iii) Let $\varepsilon=-1$ and $\alpha \geq \min (\eta, 0)$. We use again the substitution (2-3) for some $d \neq 0$. If $y_{d}$ is not constant and has a maximal point where it is positive, then (2-14) holds. Taking $d \in(0, \min (\alpha, \eta))$ if $N \geq 2$ and $\alpha>0$ and $d=-1$ if $N=1$ and $\eta=-1 \leq \alpha$, we reach a contradiction. Now suppose $w$ is regular and $\alpha>0$. Then $w^{\prime}>0$ near 0 , from Theorem 2.2, and as long as $w$ stays positive, any extremal point $r$ is a strict minimum; thus in fact $w^{\prime}>0$ on $\left[0, S_{w}\right)$. 
(iv) Let $\varepsilon=-1$ and $-p^{\prime} \leq \alpha<\min (0, \eta)$. Suppose that $w^{\prime}$ has two consecutive zeros $\rho_{1}<\rho_{2}$, and use (2-3) again with $d=\alpha$. If the function $Y_{\alpha}$ is not constant and has a maximal point $\tau$ where it is positive, we get from (2-15)

$$
Y_{\alpha}^{\prime \prime}(\tau)=(p-1)^{2}(\eta-\alpha)\left(p^{\prime}+\alpha\right) Y_{\alpha}(\tau) ;
$$

thus $Y_{\alpha}^{\prime \prime}(\tau)<0$, and $Y_{\alpha}$ has at most one zero. Next consider regular solutions: they satisfy $Y_{\alpha}=e^{(\alpha(p-1)+p) \tau}(|\alpha| a / N)(1+o(1))$ near $-\infty$, by Theorem 2.2 and (2-3). Thus $\lim _{\tau \rightarrow-\infty} Y_{\alpha}=0$; as above $Y_{\alpha}$ cannot have any extremal point, so $Y_{\alpha}$ is positive and increasing. Then $w^{\prime}<0$ from (2-3), and $w$ has at most one zero.

Remark 2.6. From (2-35) and (2-36) we see that if $0<\alpha \leq \eta$ then $y_{\alpha}$ has only minimal points on any set where it is positive, and the same conclusion holds for $Y_{\alpha}$ when $\left.-p^{\prime} \leq \alpha \leq \min (\eta, 0)\right)$.

Proposition 2.7. Let $y$ be any solution of $\left(E_{y}\right)$, linked with $w$ by (2-7), and having constant sign in a semi-interval around the point $\ln R_{w}$ or $\ln S_{w}$.

(i) If $y$ is not strictly monotone near that same point, then $R_{w}=0$ or $S_{w}=\infty$. If $y$ is not constant, then either $\varepsilon=1$ and $\delta<N<\alpha$ or $\varepsilon=-1$ and $\alpha<\delta<N$. In any case, $y$ oscillates around $\ell$.

(ii) If $y$ is strictly monotone near $\ln R_{w}$ or $\ln S_{w}$, then also $Y, \zeta, \sigma$ are monotone near the same point.

Proof. Let $s=R_{w}$ or $S_{w}$, and suppose that $y$ has constant sign near $s$. Then so does $Y$, by Remark 2.3 .

(i) At each point $\tau$ where $y^{\prime}(\tau)=0$, we have $y^{\prime \prime}(\tau) \neq 0$, and (2-16) holds with $y>0$. Suppose that $y$ is not strictly monotone near $s$. There exists a strictly monotone sequence $\left(\tau_{n}\right)$ converging to $s$ and such that $y^{\prime}\left(\tau_{n}\right)=0, y^{\prime \prime}\left(\tau_{2 n}\right)>0$, $y^{\prime \prime}\left(\tau_{2 n+1}\right)<0$. Then either $\varepsilon=1$ and $\delta<\min (\alpha, N)$, or $\varepsilon=-1$ and $\alpha<\delta<N$; and $y\left(\tau_{2 n}\right)<\ell<y\left(\tau_{2 n+1}\right)$. This cannot happen if $s$ is finite, because $y$ tends to $\infty$. It is also impossible when $\varepsilon=1$ and $\alpha \leq N$; indeed, there exist at least two points $\theta_{1}<\theta_{2}$ such that $y\left(\theta_{1}\right)=y\left(\theta_{2}\right)=\ell$ and $y \geq \ell$ on $\left(\theta_{1}, \theta_{2}\right)$, with $y^{\prime}\left(\theta_{1}\right)>0>y^{\prime}\left(\theta_{2}\right)$. Then from $(S), Y\left(\theta_{1}\right)<(\delta \ell)^{p-1}<Y\left(\theta_{2}\right)$. And from $(2-13),\left(e^{(N-\delta) \tau}(y-Y)\right)^{\prime}=$ $(N-\alpha) e^{(N-\delta) \tau} y$; and the constant $\left(\ell,(\delta \ell)^{p-1}\right)$ is also a solution of $(S)$, hence

$$
\left(e^{(N-\delta) \tau}\left(y-\ell-Y+(\delta \ell)^{p-1}\right)\right)^{\prime}=(N-\alpha) e^{(N-\delta) \tau}(y-\ell) \geq 0
$$

on $\left(\theta_{1}, \theta_{2}\right)$. A contradiction follows by integration on this interval.

(ii) Suppose $y$ strictly monotone near $s$. At any extremal point $\tau$ of $Y$, we find $Y^{\prime \prime}(\tau)=\varepsilon \alpha y^{\prime}(\tau)$ from $(2-17)$; hence $y^{\prime}(\tau) \neq 0$, and $Y^{\prime \prime}(\tau)$ has constant sign; thus $\tau$ is unique, and $Y$ is strictly monotone near $s$. 
Next consider the function $\zeta$ satisfying (2-9). If there exists $\tau_{0}$ such that $\zeta\left(\tau_{0}\right)=$ $\alpha$, then $\zeta^{\prime}\left(\tau_{0}\right)=\alpha(\alpha-\eta)$. If $\alpha \neq \eta$, then $\tau_{0}$ is unique, so $\alpha-\zeta$ has a constant sign near $s$. Then also $\zeta^{\prime \prime}(\tau)$ has constant sign at any extremal point $\tau$ of $\zeta$, from (2-18). Then $\zeta$ is strictly monotone near $s$. If $\alpha=\eta$, then $\zeta \equiv \alpha$.

Finally consider $\sigma$, which satisfies (2-10). At each point $\tau$ such that $\sigma^{\prime}(\tau)=0$, (2-19) holds and $Y$ has a constant sign. If there exists $\tau_{0}$ such that $\sigma\left(\tau_{0}\right)=\varepsilon$, then $\sigma^{\prime}\left(\tau_{0}\right)=\varepsilon(\alpha-N)$. If $\alpha \neq N$, then $\tau_{0}$ is unique, and $\sigma-\varepsilon$ has constant sign near $s$. Thus $\sigma^{\prime \prime}(\tau)$ has constant sign at any extremal point $\tau$ of $\sigma$, by (2-19), since $Y$ has constant sign near $s$. If $\alpha=N$, then $\sigma \equiv \varepsilon$.

Behavior of $w$ near 0 or $\infty$. Here we suppose $w$ is defined near 0 or $\infty$, which means the function $y$ of (2-7) is defined near $\pm \infty$. We study the behavior of $y$ and then return to $w$. First we suppose $y$ monotone, so we can assume $y>0$ near $\pm \infty$. We do not look for a priori estimates, which could be obtained by successive approximations as in [Bidaut-Véron 2006a]. Our method is based on monotonicity and L'Hospital's rule, which is much more rapid and efficient.

Proposition 2.8. Let $(y, Y)$ be any solution of $(S)$ such that $y$ is strictly monotone and $y>0$ near $s= \pm \infty$. Then $\zeta$ has a finite limit $\lambda$ near $s$, which is equal to $0, \alpha, \eta, \delta$. More precisely, we are in one of the following cases:

(i) $(y, Y)$ converges to a stationary point different from $(0,0)$. Then $\lambda=\delta$, and $\varepsilon(\delta-N)(\delta-\alpha)>0$ or $\alpha=\delta=N$.

(ii) $(y, Y)$ converges to $(0,0)$. Then

- either $\lambda=0, s=-\infty$, and $y$ is regular, or $N=1$;

- or $\lambda=\eta$; then either $(s=\infty, \delta<N)$ or $(s=\infty, \delta=N, \varepsilon(\alpha-N)<0))$ or $(s=-\infty, N<\delta)$ or $(s=-\infty, \delta=N, \varepsilon(\alpha-N)>0))$.

(iii) $\lim _{\tau \rightarrow s} y=\infty$ and $\lambda=\alpha$. Then either $(s=\infty, \alpha<\delta)$ or $(s=\infty, \alpha=$ $\delta, \varepsilon(\delta-N)<0)$ or $(s=-\infty, \delta<\alpha)$ or $(s=-\infty, \alpha=\delta, \varepsilon(\delta-N)>0)$.

Proof. From Proposition 2.7, the functions $y, Y, \sigma, \zeta$ are monotone; hence $\zeta$ has a limit $\lambda \in[-\infty, \infty]$ and $\sigma$ has a limit $\mu \in[-\infty, \infty]$, and $(y, Y)$ converges to a stationary point, or $\lim y=\infty$. Then $\lim |Y|=\infty$, since $\alpha \neq 0$ from system $(S)$. To apply the L'Hospital's rule, we consider the two quotients

$$
\frac{Y^{\prime}}{y^{\prime}}=\frac{(\delta-N) \sigma+\varepsilon(\alpha-\zeta)}{\delta-\zeta}
$$

and

$$
\begin{aligned}
\frac{\left(|Y|^{(2-p) /(p-1)} Y\right)^{\prime}}{y^{\prime}} & =\frac{\zeta(\delta-N+\varepsilon(\alpha-\zeta) / \sigma)}{(p-1)(\delta-\zeta)} \\
& =\frac{\zeta(\delta-N)+\varepsilon(\alpha-\zeta)|\zeta y|^{2-p}}{(p-1)(\delta-\zeta)}
\end{aligned}
$$


(i) First case: $\varepsilon(\delta-N)(\delta-\alpha)>0$ and $(y, Y)$ converges to the point $M_{\ell}$ defined by (2-26). Then obviously $\lambda=\delta$; or $\alpha=\delta=N$ and $\lim _{\tau \rightarrow s} y=k>0$; then $\lim _{\tau \rightarrow s} Y=(\delta k)^{p-1}$, so $\lambda=\delta$.

(ii) Second case: $(y, Y)$ converges to $(0,0)$. Then $\lambda$ is finite; indeed, if $\lambda= \pm \infty$, the quotient $(2-38)$ converges to $(N-\delta) /(p-1)$, because $|\zeta y|=|Y|^{1 /(p-1)}=o(1)$; thus $\zeta=|Y|^{(2-p) /(p-1)} Y / y$ has the same limit, from L'Hospital's rule, which is contradictory.

We next consider $N$ in relation to $\delta$. If $N<\delta$, then $(0,0)$ is a source, thus $s=$ $-\infty$. Using the eigenvectors, either $\mu=\varepsilon \alpha / N$, then $|\zeta|^{p-1}=|\mu| y^{2-p}(1+o(1))$, thus $\lambda=0$ and $w$ is regular, from Remark 2.4. Or $\mu= \pm \infty$; then $\lambda=\lambda(\delta-$ $N) /(p-1)(\delta-\zeta)$ from (2-38), thus $\lambda=0$ or $\lambda=\eta$. If $\lambda=0$, then $\zeta^{\prime} / \zeta$ converges to $-\eta$ from (2-9), and $s=-\infty$, thus necessarily $\eta<0$, which means $N=1$.

If $\delta<N$ (so $N \geq 2$ ), then $(0,0)$ is a saddle point. Thus either $s=-\infty$ and $\mu=\varepsilon \alpha / N, \lambda=0$ and $w$ is regular. Or $s=\infty, \mu= \pm \infty$, and as above, $\lambda=0$ or $\lambda=\eta$. Now if $\lambda=0$ the quotient (2-37) converges to $\mp \infty$, which is contradictory. Thus $\lambda=\eta$.

If $\delta=N$ (so $N \geq 2$ ), either $\lambda=0$, so $y^{\prime}>0, s=-\infty$, and $\mu=\varepsilon \alpha / N$ by (2-38); or else $\lambda>0$, in which case $\lambda=N=\eta$ from (2-38). Moreover if $s=\infty$, then $\varepsilon(\alpha-N)<0$; if $s=-\infty$, then $\varepsilon(\alpha-N)>0$. Indeed $(\varepsilon y-Y)^{\prime}=\varepsilon(N-\alpha) y$ and $y-\varepsilon Y$ converges to 0 ; thus if $s=\infty$ and $\varepsilon(N-\alpha) \geq 0$, or $s=-\infty$ and $\varepsilon(N-\alpha) \leq 0$, then $\mu \leq \varepsilon$, but $\mu=\infty$, we reach again a contradiction.

(iii) Third case: $y$ tends to $\infty$. If $s=\infty$, then $y^{\prime}>0$, thus $\zeta<\delta$; if $s=-\infty$, then $\zeta>\delta$. If $\lambda= \pm \infty$, then the quotient (2-38) converges to $\varepsilon \infty$; thus $\lambda=\varepsilon \infty$ and $s=-\varepsilon \infty$. In any case, $\zeta^{\prime}<0$, so $|\mu| \leq 1 /(p-1)$ by (2-9), and $\mu=\varepsilon$ by (2-37); thus $Y^{\prime}=-\varepsilon|Y|^{(2-p) /(p-1)} Y(1+o(1))$, and we reach a contradiction by integration. Thus $\lambda$ is finite; moreover $\lambda \neq 0$ for otherwise we would have $\mu=0$, seeing that $\sigma=|\zeta y|^{p-2} \zeta$; but $\mu=\alpha / \delta$ by (2-37).

If $\alpha \neq \delta$, then $\lambda=\alpha$ or $\delta$, by (2-38). In turn $\sigma=|\lambda y|^{p-2} \lambda(1+o(1))$, thus $\mu=0$. From (2-37), necessarily $\lambda=\alpha$. And if $s=\infty$, then $y^{\prime}>0$, thus $\zeta<\delta$, thus $\alpha<\delta$. If $s=-\infty$, then similarly $\alpha>\delta$.

If $\alpha=\delta$, then $\lambda=\alpha=\delta \neq N$, and $\varepsilon(\delta-N)(\delta-\zeta)<0$ from (2-38); thus if $s=\infty$, then $\varepsilon(\delta-N)<0$ since $\zeta<\delta$; if $s=-\infty$, then $\varepsilon(\delta-N)>0$.

Next we improve Proposition 2.8 by giving a precise behavior of $w$ in any case:

Proposition 2.9. We keep the assumptions of Proposition 2.8.

(i) If $\lambda=\alpha \neq \delta$, then $\lim r^{\alpha} w=L>0$ (near 0 , or $\left.\infty\right)$.

(ii) If $\lambda=\eta>0, \eta \neq N$, then $\lim r^{\eta} w=c>0$. 
(iii) If $\lambda=\alpha=\delta \neq N$, then

$$
\lim r^{\delta}(\ln r)^{-1 /(2-p)} w=\kappa:=\left((2-p) \delta^{p-1}|N-\delta|\right)^{1 /(2-p)} .
$$

(iv) If $\lambda=\eta=N=\delta \neq \alpha$, then

$$
\lim r^{N}(\ln r)^{(N+1) / 2} w=\rho:=\frac{1}{N}\left(\frac{N(N-1)}{2|\alpha-N|}\right)^{(N+1) / 2} .
$$

(v) If $N=1, \lambda=\eta=-1$ or $\lambda=0$ (near 0$)$ then

$$
\lim _{r \rightarrow 0} w=a \in \mathbb{R}, \quad \lim _{r \rightarrow 0} w^{\prime}=b ;
$$

and $b \neq 0$; moreover, $a=0$ (hence $b>0$ ) if and only if $\lambda=-1$.

Proof. (i) Let $\lambda=\alpha \neq \delta$. From (2-8) we have $r w^{\prime}(r)=-\alpha w(r)(1+O(1))$. Next we apply Proposition 2.8, and are led to two cases:

If $s=\infty$ and $\alpha<\delta$, then for any $\gamma>0$ we have $w=O\left(r^{-\alpha+\gamma}\right)$ and $1 / w=$ $O\left(r^{\alpha+\gamma}\right)$ near $\infty$ and $w^{\prime}=O\left(r^{-\alpha-1+\gamma}\right)$. Then $J_{\alpha}^{\prime}(r)=O\left(r^{\alpha(2-p)-p-1+\gamma}\right)$, so $J_{\alpha}^{\prime}$ is integrable, hence $J_{\alpha}$ has a $\operatorname{limit} L$, and $\lim r^{\alpha} w=L$, seeing that $J_{\alpha}(r)=$ $r^{\alpha} w(1+o(1))$. If $L=0$, then $r^{\alpha} w=O\left(r^{\alpha(2-p)-p+\gamma}\right)$, which contradicts the estimate of $1 / w=O\left(r^{\alpha+\gamma}\right)$ for $\gamma$ small enough. Thus $L>0$.

Otherwise, we have $s=-\infty$ and $\delta<\alpha$; hence $\lim _{\tau \rightarrow s} y=\infty, w=O\left(r^{-\alpha-\gamma}\right)$, $1 / w=O\left(r^{\alpha-v}\right), w^{\prime}=O\left(r^{-\alpha-1-\gamma}\right)$ near 0 , and $J_{\alpha}^{\prime}(r)=O\left(r^{\alpha(2-p)-p-1-\gamma}\right)$. Thus $J_{\alpha}^{\prime}$ is still integrable; hence $\lim r^{\alpha} w=L \geq 0$. If $L=0$, then $r^{\alpha} w=O\left(r^{\alpha(2-p)-p-\gamma}\right)$, which contradicts the estimate of $1 / w$. Therefore we again obtain $L>0$.

(ii) Let $\lambda=\eta>0, \eta \neq N$. From Proposition 2.8, either $s=\infty, \delta<N$ or $s=-\infty$, $N<\delta$. As above we get $w=O\left(r^{-\eta \pm \gamma}\right)$ and $1 / w=O\left(r^{\eta \pm \gamma}\right)$ near $\infty$ or 0 . Here we make the substitution (2-3) with $d=\eta$. We find $y_{\eta}=O\left(e^{ \pm \gamma \tau}\right), 1 / y_{\eta}=O\left(e^{ \pm \gamma \tau}\right)$, $y_{\eta}^{\prime}=O\left(e^{ \pm \gamma \tau}\right)$, thus $Y_{\eta}=O\left(e^{ \pm \gamma \tau}\right)$, and from (2-4), $Y_{\eta}^{\prime}=O\left(e^{ \pm \gamma \tau}\right)$. Substituting in (2-4), we deduce $Y_{\eta}^{\prime}=O\left(e^{(2-p)((\delta-\eta) \pm \gamma) \tau}\right)$. When $s=\infty$, then $\delta<\eta$, when $s=$ $-\infty$, then $\delta>\eta$ from (1-2). In any case, $Y_{\eta}^{\prime}$ is integrable, hence $Y_{\eta}$ has a limit $k$, and $Y_{\eta}-k=O\left(e^{(2-p)((\delta-\eta) \pm \gamma) \tau}\right)$. Now $\left(e^{-\eta \tau} y_{\eta}\right)^{\prime}=-e^{-\eta \tau} Y_{\eta}^{1 /(p-1)}$, thus $y_{\eta}$ has a limit $c=k^{1 /(p-1)} / \eta$; in other words, $\lim r^{\eta} w=c$. If $c=0$, then $Y_{\eta}=O\left(e^{(2-p)((\delta-\eta) \pm \gamma) \tau}\right)$, $y_{\eta}=O\left(e^{((2-p)((\delta-\eta) \pm \gamma) /(p-1)) \tau}\right)$, which contradicts $1 / y_{\eta}=O\left(e^{\gamma \tau}\right)$ for $\gamma$ small.

(iii) Now suppose $\lambda=\alpha=\delta \neq N$. Then either $s=\infty$ and $\varepsilon(\delta-N)<0$ or $s=-\infty$ and $\varepsilon(\delta-N)>0$; moreover, $\lim _{\tau \rightarrow s} y=\infty$. Then $Y=(\delta y)^{p-1}(1+o(1))$, and $\mu=0$; hence $y-\varepsilon Y=y(1+o(1))$, and from $(2-13)$,

$$
(y-\varepsilon Y)^{\prime}=\varepsilon(N-\delta) Y=\varepsilon(N-\delta) \delta^{p-1}(y-\varepsilon Y)^{p-1}(1+o(1)) .
$$

Then $y=\left(|N-\delta| \delta^{p-1}(2-p)|\tau|\right)^{1 /(2-p)}(1+o(1))$, which is equivalent to (2-39) by $(2-7)$. 
(iv) Let $\lambda=\eta=N=\delta \neq \alpha$. Then either $s=\infty$ and $\varepsilon(\alpha-N)<0$ or $s=-\infty$ and $\varepsilon(\alpha-N)>0$; moreover, $\lim _{\tau \rightarrow s} y=0$. Then $Y=(N y)^{p-1}(1+o(1))$ and $\mu=\infty$, so $Y-\varepsilon y=Y(1+o(1))$, and from (2-13) we have

$$
(Y-\varepsilon y)^{\prime}=\varepsilon(\alpha-N) y=\varepsilon(\alpha-N) N^{-1}(Y-\varepsilon y)^{1 /(p-1)}(1+o(1)) .
$$

Hence $y=c|\tau|^{-(N+1) / 2}(1+o(1))$ with $c=N^{-1}(N(N-1) / 2|\alpha-N|)^{(N+1) / 2}$, and (2-40) follows from (2-7).

(v) Let $\lambda=0$. Then also $r w^{\prime}=o(w)$; thus by integration we get $w+\left|w^{\prime}\right|=O\left(r^{-k}\right)$ for any $k>0$. Then $J_{1}^{\prime}$ is integrable, so $J_{1}$ has a limit at 0 , and $\lim _{r \rightarrow 0} r w=0$. Therefore $\lim _{r \rightarrow 0} w^{\prime}=b \in \mathbb{R}$ and $\lim _{r \rightarrow 0} w=a \geq 0$. Then $b \neq 0$, since regular solutions satisfy (2-33), and $a \neq 0$, since $a=0$ would imply $w=-b r(1+o(1))$, $\zeta=-1$. If $\lambda=\eta=-1$, then from (2-8), $w$ is nondecreasing, so it has a limit $a \geq 0$ at 0 , leading to $w^{\prime}=-a \lambda r^{-1}(1+o(1))$, and by integration $a=0$. And $\left(\left(w^{\prime}\right)^{p-1}\right)^{\prime}=\varepsilon(1-\alpha) w(1+o(1))$, so $w^{\prime}$ has a limit $b \neq 0$.

Next we consider the cases where $y$ is not monotone and possibly changes sign. Proposition 2.10. Assume $\varepsilon=1$.

(i) Suppose that $N \leq \delta<\alpha$, or $N<\delta \leq \alpha$. Then any solution $y$ has a infinite number of zeros near $\infty$.

(ii) Suppose that $y$ has a infinite number of zeros near $\pm \infty$. Then either

$$
N<\alpha<\delta \text { and }|y|<\ell \text { and }|Y|<(\delta \ell)^{p-1} \text { near } \pm \infty,
$$

or $N<\delta=\alpha$, or $\max (\delta, N, \eta)<\alpha$. If moreover $\delta<N<\alpha$, then $|y|$ exceeds $\ell$ at its extremal points and $|Y|$ exceeds $(\delta \ell)^{p-1}$ at its extremal points.

Proof. (i) Suppose the conclusion does not hold. Then for example $y>0$ for large $\tau$; and $y$ is monotone, from Proposition 2.7(i). Applying Proposition 2.8 with $s=\infty$, we reach a contradiction.

(ii) Suppose that $y$ is oscillating around 0 near $\pm \infty$. Then from $(2-16)$, at the extremal points,

$$
|y(\tau)|^{2-p}(\delta-\alpha)<(\delta-N) \delta^{p-1},
$$

and the inequality is strict, because in case of equality, $y$ is constant by uniqueness. Similarly $Y$ is oscillating around 0 , and at the extremal points one finds, from $(2-17)$,

$$
|Y(\tau)|^{(2-p) /(p-1)}(\delta-\alpha)<(\delta-N) \delta .
$$

Then $\max (N, \eta)<\alpha$, thanks to Proposition 2.5; and the conclusions follow from (2-42) and (2-43). 
We can complete these results according to the sign of $\delta-N / 2$ :

Proposition 2.11. Suppose that $\varepsilon(\delta-N / 2) \leq 0$. Then any solution $y$ has a finite number of zeros near $\ln R_{w}$ or $\ln S_{w}$. If $y$ is defined near $\pm \infty$ and nonmonotone, then $(y, Y)$ converges to $\pm M_{\ell}$. There is no cycle or homoclinic orbit in $\mathbb{R}^{2}$.

Proof. (i) Suppose that $y$ has an infinity of zeros. Then $R_{w}=0$ or $S_{w}=\infty$, and there exists a strictly monotone sequence $\left(r_{n}\right)$ of consecutive zeros of $w$, converging to 0 or $\infty$. Since $\varepsilon(\delta-N / 2) \leq 0$, the energy function $V$ defined in (2-24) is nonincreasing. We claim that $V$ is bounded. This is not easy to prove; we define for the purpose a function

$$
U(r)=r^{N}\left(\frac{1}{2} w^{2}+\varepsilon r^{-1}\left|w^{\prime}\right|^{p-2} w^{\prime} w\right)=e^{(N-2 \delta) \tau} y\left(\frac{1}{2} y-\varepsilon Y\right) .
$$

Then

$$
U^{\prime}(r)=r^{N-1}\left(\left(\frac{1}{2} N-\alpha\right) w^{2}+\varepsilon\left|w^{\prime}\right|^{p}\right)=e^{(N-1-2 \delta) \tau}\left(\left(\frac{1}{2} N-\alpha\right) y^{2}+\varepsilon|Y|^{p^{\prime}}\right) .
$$

If $\varepsilon=1$, then $\delta \leq N / 2<N<\alpha$. If $\varepsilon=-1$, then $\alpha<0$, by Proposition 2.10. Then $U\left(r_{n}\right)=0$ and $\varepsilon U^{\prime}\left(r_{n}\right)>0$. Therefore there exists another sequence $\left(s_{n}\right)$ such that $s_{n} \in\left(r_{n}, r_{n+1}\right), U\left(s_{n}\right)=0$, and $\varepsilon U^{\prime}\left(s_{n}\right) \leq 0$. At the point $\tau_{n}=e^{s_{n}}$ we find $2^{1-p^{\prime}} y^{2 p^{\prime}}=2|Y|^{p^{\prime}} \leq \varepsilon(2 \alpha-N) y^{2}$, so $\left(y\left(\tau_{n}\right), Y\left(\tau_{n}\right)\right)$ is bounded. Hence $\left(V\left(\tau_{n}\right)\right)$ is bounded, so $V$ is bounded near $\pm \infty$. Therefore $V$ has a finite limit $\chi$, and $Y$ and $Y^{\prime}$ are bounded because $\varepsilon(\alpha-\delta)>0$; in turn, $(y, Y)$ is bounded. Otherwise $(0,0)$ and $\pm M_{\ell}$ are not in the limit set at $\pm \infty$, since $(0,0)$ is a saddle point, and $\pm M_{\ell}$ is a source or a sink. Then the trajectory has a limit cycle, and there exists a periodic solution $(y, Y)$. The corresponding function $V$ is periodic and monotone, hence constant; then $V^{\prime} \equiv 0$ implies that $Y$ is constant and hence also $y$, by $(S)$. But this is a contradiction.

(ii) Suppose that $y$ is positive near $\pm \infty$, and nonmonotone. If $\varepsilon=1$, then $\delta \leq$ $N / 2<N<\alpha$; if $\varepsilon=-1$, then $\alpha<\delta<N$, by Proposition 2.7, and $y$ oscillates around $\ell$. There exists a sequence of minimal points $\left(\tau_{n}\right)$, where $y\left(\tau_{n}\right)<\ell$, and $\left|Y\left(\tau_{n}\right)\right|=$ $\delta y\left(\tau_{n}\right)$; thus again $\left(y\left(\tau_{n}\right), Y\left(\tau_{n}\right)\right)$ is bounded, and as above $(y, Y)$ is bounded. The trajectory has no limit cycle, and hence converges to $M_{\ell}$. Finally, if there is an homoclinic orbit, then $\mathscr{T}_{r}$ is homoclinic. Then $\lim _{\tau \rightarrow-\infty} V=\lim _{\tau \rightarrow \infty} V=0$; hence $V \equiv 0$, and as above $(y, Y)$ is constant, so $(y, Y) \equiv(0,0)$, again a contradiction.

Proposition 2.12. If $y$ is not monotone near $\varepsilon \infty$ (positive or changing sign), then $y$ and $Y$ are bounded.

Proof. From Proposition 2.11, it follows that $\varepsilon(\delta-N / 2)>0$. When $\varepsilon=1$, and $y$ is changing sign and $N<\alpha<\delta$, then $|y|$ is bounded by $\ell$ from above. Apart from this case, if $y$ is changing sign, then $\varepsilon(\alpha-\delta)>0$, from Proposition 2.11. If $y$ stays positive, either $\varepsilon=1, \delta<\min (\alpha, N)$, or $\varepsilon=-1, \alpha<\delta<N$, by Proposition 2.7. 
In any case $\varepsilon(\alpha-\delta)>0$. Here we use the energy function $W$ defined by (2-21). We can write $\mathscr{W}(y, Y)$ in the form

$$
\mathscr{W}(y, Y)=\varepsilon(F(y, Y)+G(y))
$$

with

$$
F(y, Y)=\frac{|Y|^{p^{\prime}}}{p^{\prime}}-\delta y Y+\frac{|\delta y|^{p}}{p}, \quad G(y)=\frac{(\delta-N) \delta^{p-1}}{p}|y|^{p}+\frac{\varepsilon(\alpha-\delta)}{2} y^{2} .
$$

Observe that $F(y, Y) \geq 0$, so $\varepsilon^{q} W(y, Y) \geq G(y)>0$ for large $|y|$. Then $W^{\prime}(\tau) \leq 0$ whenever $(y(\tau), Y(\tau)) \notin \mathscr{Y}_{\mathscr{L}}$, where $\mathscr{S}_{\mathscr{L}}$ is given in (2-22). Let $\tau_{0}$ be arbitrary in the interval of definition of $y$. Since $\mathscr{S}_{\mathscr{L}}$ is bounded, there exists $k>0$ large enough that $\varepsilon W(\tau) \leq k$ for any $\tau$ such that $\varepsilon\left(\tau-\tau_{0}\right) \geq 0$ and $(y(\tau), Y(\tau)) \in \mathscr{Y}_{\mathscr{L}}$, and we can choose $k>W\left(\tau_{0}\right)$. Then $\varepsilon W(\tau) \leq k$ for $\varepsilon\left(\tau-\tau_{0}\right) \geq 0$; hence $y$ and $Y$ are bounded near $\varepsilon \infty$.

Further sign properties. We can improve Proposition 2.5 using Propositions 2.8 and 2.9:

Proposition 2.13. Assume $\varepsilon=1,-\infty<\alpha \leq \delta$ and $\alpha<N$. Then all regular solutions have constant sign, $y$ is strictly monotone and $\lim _{\tau \rightarrow \infty} \zeta=\alpha$. Any solution has at most one zero, and $\lim _{\tau \rightarrow \infty} \zeta=\alpha$.

Proof. Regular solutions have constant sign by Proposition 2.5. Moreover $J_{N}$ is increasing from 0; thus it is positive for $r>0$, which means $Y<y$. And $y$ is monotone near $\infty$, by Proposition 2.7. From Proposition 2.8, we have three possibilities: either $\alpha<N<\delta$ and $\lim _{\tau \rightarrow \infty} \zeta=\delta$, in which case $\lim _{\tau \rightarrow \infty} Y / y=$ $(\delta-\alpha) /(\delta-N)>1$, which is impossible; or $\delta \leq N$ and $\lim _{\tau \rightarrow \infty} \zeta=\eta \geq N$, in which case $\lim _{\tau \rightarrow \infty} Y / y=\infty$, which is also contradictory, or finally $\lim _{\tau \rightarrow \infty} \zeta=\alpha$. Moreover $y$ is increasing on $\mathbb{R}$ from 0 to $\infty$; indeed, if $y$ has a local maximum for some $\tau$, we get $\alpha<N<\delta$ and $y(\tau) \leq \ell$ from (2-16), and moreover $\ell<\delta^{(p-1) /(2-p)}$; but $\delta y(\tau)=Y(\tau)^{1 /(p-1)}<y(\tau)^{1 /(p-1)}$, which is contradictory.

For the second statement, we see from Proposition 2.5 that any solution $w \not \equiv 0$ has at most one zero. If $w\left(r_{1}\right)=0$ and, say, $w>0$ on $\left(r_{1}, \infty\right)$, we get $w^{\prime}\left(r_{1}\right)>0$; thus $J_{N}(r) \geq J_{N}\left(r_{1}\right)>0$ for $r \geq r_{1}$, and we conclude as above.

Proposition 2.14. Assume $\varepsilon=-1$.

(i) If $\alpha<0$ and $N \leq \delta$, all regular solutions have at least one zero.

(ii) If $0<\alpha$, all regular solutions have constant sign and satisfy $S_{w}<\infty$.

(iii) If $-p^{\prime}<\alpha<\min (0, \eta)$, all regular solutions have precisely one zero and $S_{w}<\infty$. 
Proof. (i) Let $\alpha<0$ and $N \leq \delta$. Since $\varepsilon \alpha>0$, the trajectory $\mathscr{T}_{r}$ starts in $\mathscr{2}_{1}$. Suppose that $y$ stays positive. Then $\mathscr{T}_{r}$ stays in $2_{1}$, from Remark 2.3. If $N \leq \delta$, then $y$ is monotone, since it can only have minimal points, from $(2-16)$; and $(0,0)$ is the only stationary point. Then $\lim _{\tau \rightarrow \infty} y=\infty$, and $\lim _{\tau \rightarrow \infty} \zeta=\alpha<0$ from Proposition 2.8; thus $(y, Y)$ is in $2_{4}$ for large $\tau$, which is impossible.

(ii) Let $0<\alpha$. Then $\varepsilon \alpha<0$, so that $\mathscr{T}_{r}$ starts in 24 . Moreover $y>0$ on $\mathbb{R}$, by Proposition 2.5. And $\mathscr{T}_{r}$ stays in $2_{4}$, by Remark 2.1(i) on page 211. Thus $y^{\prime}=$ $\delta y+|Y|^{1 /(p-1)}>0$. If $S_{w}=\infty$, we see from Proposition 2.8 that $\lim _{\tau \rightarrow \infty} \zeta=\alpha>0$; hence $(y, Y)$ ends up in $\mathscr{2}_{1}$, which is false. Then $S_{w}<\infty$.

(iii) Let $-p^{\prime}<\alpha<\min (0, \eta)$. Then $\mathscr{T}_{r}$ starts in $\mathscr{2}_{1}$. By Proposition 2.5, $Y_{\alpha}$ stays positive, $\mathscr{T}_{r}$ stays in $\mathscr{2}_{1} \cup \mathscr{2}_{2}$, and $Y_{\alpha}$ is increasing:

$$
Y_{\alpha}^{\prime}=-(p-1)(\eta-\alpha) Y_{\alpha}+e^{(p-(2-p) \alpha) \tau}\left(Y_{\alpha}^{1 /(p-1)}-\alpha y_{\alpha}\right)>0 .
$$

Suppose that $S_{w}=\infty$. Then $\lim _{\tau \rightarrow \infty} Y_{\alpha}(\tau) \geq C>0$, so $r^{\alpha+1} w^{\prime}(r) \leq-C^{1 /(p-1)}$ for large $r$, and, by integration, $r^{\alpha} w(r) \leq-C^{1 /(p-1)} / 2$. In particular, we obtain from (2-3) that $\lim _{\tau \rightarrow \infty} y=-\infty$. From Propositions 2.7, 2.8, and 2.9, it follows that $\lim _{r \rightarrow \infty} r^{\alpha} w=L<0$; thus $\lim _{\tau \rightarrow \infty} Y_{\alpha}(\tau)=(\alpha L)^{p-1}$. And there exists a unique $\tau_{0}$ such that $y_{\alpha}\left(\tau_{0}\right)=0$, by Remark 2.1(i). But

$$
\begin{aligned}
Y_{\alpha}^{\prime \prime}(\tau) & -(p-1)^{2}(\eta-\alpha)\left(\alpha+p^{\prime}\right) Y_{\alpha} \\
& =\frac{Y_{\alpha}^{\prime}}{Y_{\alpha}}\left(\frac{1}{p-1} e^{(p-(2-p) \alpha) \tau} Y_{\alpha}^{1 /(p-1)}-(p-1)\left(\eta-2 \alpha-p^{\prime}\right) Y_{\alpha}\right) \\
& \geq \frac{Y_{\alpha}^{\prime}}{Y_{\alpha}}\left(\frac{\alpha}{p-1} e^{(p-(2-p) \alpha) \tau} y_{\alpha}+(\eta-\alpha)(2-p)+(p-1)\left(\alpha+p^{\prime}\right) Y_{\alpha}\right) .
\end{aligned}
$$

Thus $Y_{\alpha}^{\prime \prime}(\tau)>0$ for any $\tau \geq \tau_{0}$, an impossibility. Then $S_{w}<\infty, \lim _{\tau \rightarrow \ln } S_{w} Y / y=$ -1 , and $y$ has a zero.

\section{Behavior of $w$ near $R_{w}>0$ or $S_{w}<\infty$.}

Proposition 2.15. Let $w$ be any solution of $\left(E_{w}\right)$ with a reduced domain (so either $\varepsilon=1$ and $R_{w}>0$ or $\varepsilon=-1$ and $\left.S_{w}<\infty\right)$. Let $s=R_{w}$ or $S_{w}$. Then

(2-47) $\lim _{r \rightarrow s}|r-s|^{(p-1) /(2-p)} s^{1 /(2-p)} w= \pm\left(\frac{p-1}{2-p}\right)^{\frac{p-1}{2-p}} \quad$ and $\quad \lim _{\tau \rightarrow \ln s} \sigma=\varepsilon$.

Proof. From Proposition 2.5, we can suppose that $\varepsilon w$ is decreasing near $s$ and $\lim _{r \rightarrow s} w=\infty$; thus $y>0, \varepsilon Y>0$ near $\ln s$, and $\lim _{\tau \rightarrow \ln s} y=\infty$. Also, $\sigma$ is monotone near $\ln s$, by Proposition 2.7; thus it has a limit $\mu$ such that $\varepsilon \mu \in[0, \infty]$. Suppose that $\mu=0$. Then $Y=o(y)=o(y-\varepsilon Y)$; from (2-13) we get

$$
(y-\varepsilon Y)^{\prime}=(\delta-\alpha)(y-\varepsilon Y)+\varepsilon(N-\alpha) Y=(\delta-\alpha+o(1))(y-\varepsilon Y),
$$


so $y$ cannot blow up in finite time. In the same way, if $\mu=\infty$, then $y=o(\varepsilon Y)=$ $o(\varepsilon Y-y)$, and

$$
(y-\varepsilon Y)^{\prime}=(\delta-N)(y-\varepsilon Y)+(N-\alpha) y=(\delta-N+o(1))(y-\varepsilon Y),
$$

again leading to a contradiction; thus $\varepsilon \mu \in(0, \infty)$. Therefore $\lim _{\tau \rightarrow \ln } R_{w} \zeta=\varepsilon \infty$ and $\mu=\varepsilon$ from (2-37); then $w^{\prime} w^{-1 /(p-1)}+(\varepsilon+o(1)) r^{1 /(p-1)}=0$, and (2-47) holds.

\section{More information on stationary points.}

The Hopf bifurcation point. When $\varepsilon(\delta-N / 2)>0$, a Hopf bifurcation appears at the critical value $\alpha=\alpha^{*}$ given by (1-11). Then some cycles do appear near $\alpha^{*}$, by the Poincaré-Andronov-Hopf theorem; see [Hale and Koçak 1991, p. 344]. We get more precise results by using the Lyapunov test for a weak sink or source; it requires an expansion up to the order 3 near $M_{\ell}$, in a suitable basis of eigenvectors, where the linearized problem has a rotation matrix.

Theorem 2.16. Let $\varepsilon(\delta-N / 2)>0$.

(i) Suppose $\varepsilon=1$. If $\alpha=\alpha^{*}$, then $M_{\ell}$ is a weak source. If $\alpha<\alpha^{*}$ with $\alpha^{*}-\alpha$ small enough, there exists a unique limit cycle in $2_{1}$ attracting at $-\infty$.

(ii) Suppose $\varepsilon=-1$. If $\alpha=\alpha^{*}$, then $M_{\ell}$ is a weak sink. If $\alpha>\alpha^{*}$ with $\alpha-\alpha^{*}$ small enough, there exists a unique limit cycle in $2_{1}$, attracting at $\infty$.

Proof. The eigenvalues are given by $\lambda_{1}=-i b, \lambda_{2}=i b$, with $b=\sqrt{p^{\prime}(N-\delta)}$. From (2-29) we get

$$
v\left(\alpha^{*}\right)=2 \delta-N=\frac{\delta(N-\delta)}{(p-1)\left(\alpha^{*}-\delta\right)}=\frac{\varepsilon(\delta \ell)^{2-p}}{(p-1)} .
$$

First we make the substitution (2-27) as above, which leads to (2-28). The function $\Psi$ defined in (2-29) has an expansion near $t=0$ of the form

$$
\Psi(\vartheta)=B_{2} \vartheta^{2}+B_{3} \vartheta^{3}+\cdots,
$$

where

$$
B_{2}=\frac{(2-p)(\delta \ell)^{3-2 p}}{2(p-1)^{2}}, \quad B_{3}=\frac{(2-p)(3-2 p)(\delta \ell)^{4-3 p}}{6(p-1)^{6}}=\frac{2(3-2 p) B_{2}^{2}}{3(2-p) v\left(\alpha^{*}\right)} .
$$

Next we make the substitution

$$
\tau=-\theta / b, \quad \bar{y}(\tau)=\varepsilon v(\alpha) x_{1}(\theta), \quad \bar{Y}(\tau)=\delta x_{1}(\theta)+b x_{2}(\theta),
$$

and obtain

$$
x_{1}^{\prime}(\theta)=x_{2}+\frac{\varepsilon}{b v(\alpha)} \Psi\left(\delta x_{1}+b x_{2}\right), \quad x_{2}^{\prime}(\theta)=-x_{1}-\frac{\varepsilon(N-\delta)}{b^{2} v(\alpha)} \Psi\left(\delta x_{1}+b x_{2}\right) .
$$


We write the expansion of order 3 in the form

$x_{1}^{\prime}=x_{2}+\varepsilon\left(a_{2,0} x_{1}^{2}+a_{1,1} x_{1} x_{2}+a_{0,2} x_{2}^{2}+a_{3,0} x_{1}^{3}+a_{2,1} x_{1}^{2} x_{2}+a_{1,2} x_{1} x_{2}^{2}+a_{0,3} x_{2}^{3}+\cdots\right)$, $x_{1}^{\prime}=-x_{1}+\varepsilon\left(b_{2,0} x_{1}^{2}+b_{1,1} x_{1} x_{2}+b_{0,2} x_{2}^{2}+b_{3,0} x_{1}^{3}+b_{2,1} x_{1}^{2} x_{2}+b_{1,2} x_{1} x_{2}^{2}+b_{0,3} x_{2}^{3}+\cdots\right)$,

and we compute the Lyapunov coefficient

$$
\begin{aligned}
L_{C}=\varepsilon\left(3 a_{3,0}+\right. & \left.a_{1,2}+b_{2,1}+3 b_{0,3}\right) \\
& \quad-a_{2,0} a_{1,1}+b_{1,1} b_{0,2}-2 a_{0,2} b_{0,2}-a_{0,2} a_{1,1}+2 a_{2,0} b_{2,0}+b_{1,1} b_{2,0} .
\end{aligned}
$$

After simplification, we obtain

$$
\begin{aligned}
\frac{(2-p) b v(\alpha)^{2}}{2 B_{2}^{2}\left(\delta^{2}+b^{2}\right)} L_{C} & =(N-2 \delta)(1-\varepsilon(3-2 p)) \\
& =\left\{\begin{array}{ll}
2(N-2 \delta)(p-1)<0 & \text { if } \varepsilon=1, \\
2(N-2 \delta)(2-p)>0 & \text { if } \varepsilon=-1,
\end{array} .\right.
\end{aligned}
$$

The nature of $M_{\ell}$ follows from [Hubbard and West 1995, p. 292], taking into account that $\theta$ has opposite sign from $\tau$. If $\varepsilon=1, M_{\ell}$ is a week source, and there exists a small limit cycle attracting at $-\infty$ for all $\alpha$ near $\alpha^{*}$ such that $M_{\ell}$ is a sink; this means that $\alpha<\alpha^{*}$. If $\varepsilon=-1, M_{\ell}$ is a weak sink and there exists a small limit cycle attracting at $\infty$ for all $\alpha$ near $\alpha^{*}$ such that $M_{\ell}$ is a source; this means $\alpha^{*}<\alpha$.

Node points or spiral points. When the system $(S)$ has three stationary points, and $M_{\ell}$ is a source or a sink (so $\delta<N$ ), it is interesting to know if $M_{\ell}$ is a node point. When $\alpha^{*}$ exists, it is a spiral point, by (2-30).

If $\varepsilon=1$, we see from (2-31) that $M_{\ell}$ is a node point when $\delta \leq N / 2-\sqrt{p^{\prime}(N-\delta)}$ or $\delta>N / 2-\sqrt{p^{\prime}(N-\delta)}$ and $\alpha \leq \alpha_{1}$, or $\delta>N / 2+\sqrt{p^{\prime}(N-\delta)}$ and $\alpha_{2} \leq \alpha$, where

$$
\begin{aligned}
& \alpha_{1}=\delta+\frac{\delta(N-\delta)}{(p-1)\left(2 \delta-N+2 \sqrt{p^{\prime}(N-\delta)}\right)}, \\
& \alpha_{2}=\delta+\frac{\delta(N-\delta)}{(p-1)\left(2 \delta-N-2 \sqrt{p^{\prime}(N-\delta)}\right)}
\end{aligned}
$$

If $\varepsilon=-1, M_{\ell}$ is a node when $\delta \geq N / 2+\sqrt{p^{\prime}(N-\delta)}$, or $\delta<N / 2+\sqrt{p^{\prime}(N-\delta)}$ and $\alpha_{2} \leq \alpha$, or $\delta<N / 2-\sqrt{p^{\prime}(N-\delta)}$ and $\alpha \leq \alpha_{1}$. In any case $\alpha_{1}<\alpha_{2}$.

Remarks 2.17. (i) Let $\varepsilon=1$. One can verify that $N \leq \alpha_{1}$ and that $N=\alpha_{1}$ if and only if $N=\delta /(p-1)=p^{\prime} /(2-p)$. Also $\alpha_{1}<\eta$ if and only if $\delta^{2}+(7-N) \delta+N>0$, which is true for $N \leq 14$, but not always. 
(ii) Let $\varepsilon=-1$. It is easy to see that $\alpha_{2} \leq 0$ and that $\alpha_{2}=0$ if and only if $N(2-p)=\delta$, or equivalently $N=p /(2-p)^{2}$. Also $\alpha_{2}>-p^{\prime}$ if and only if $\delta^{2}+7 \delta-8 N<0$, which is true for $\delta<N / 2<9$, but not always.

Nonexistence of cycles. If the system $(S)$ admits a cycle $\mathcal{O}$ in $\mathbb{R}^{2}$, then $\mathcal{O}$ surrounds at least one stationary point. If it surrounds $(0,0)$, the corresponding solutions $y$ are not of constant sign. If it only surrounds $M_{\ell}$, then it stays in $\mathscr{2}_{1}$, so $y$ stays positive. Indeed $\alpha \neq 0$ from (1-9), and 0 cannot intersect $\{(\varphi, 0), \varphi>0\}$ at two points, and similarly $\{(0, \xi), \xi>0\}$, by Remark 2.1(i) on page 211 .

For suitable values of $\alpha, \delta, N$, we can show that cycles cannot exist, by using Bendixson's criterion or the Poincare map. Writing $(S)$ under the form

$$
y^{\prime}=f_{1}(y, Y), \quad Y^{\prime}=f_{2}(y, Y),
$$

we obtain

$$
\frac{\partial f_{1}}{\partial y}(y, Y)+\frac{\partial f_{2}}{\partial Y}(y, Y)=2 \delta-N-\varepsilon|Y|^{(2-p) /(p-1)} .
$$

For example, as a direct consequence of Bendixson's criterion, if $\varepsilon(\delta-N / 2)<0$, we find again the nonexistence of any cycle in $\mathbb{R}^{2}$, which was obtained in Proposition 2.11. Now we consider cycles in $2_{1}$.

First we extend to system $(S)$ a general property of quadratic systems, proved in [Chicone and Tian 1982], stating that there cannot exist a closed orbit surrounding a node point. Note that the restriction of our system to $2_{1}$ is quadratic if $p=\frac{3}{2}$.

Theorem 2.18. Let $\delta<N$ and $\varepsilon(\delta-\alpha)<0$. When $M_{\ell}$ is a node point, there is no cycle or homoclinic orbit in $2_{1}$.

Proof. We use the linearization (2-27), (2-28), (2-29). Consider the line $L$ with equation $A \bar{y}+\bar{Y}=0$, where $A$ is a real parameter. The points of $L$ are in $2_{1}$ whenever $-(\delta \ell)^{p-1}<\bar{Y}$ and $-\ell<\bar{y}$. As in [Chicone and Tian 1982], we study the orientation of the vector field along $L$ : we find

$$
A \bar{y}^{\prime}+\bar{Y}^{\prime}=\left(\varepsilon v(\alpha) A^{2}+(N+v(\alpha)) A+\varepsilon \alpha\right) \bar{y}-(A+\varepsilon) \Psi(\bar{Y}) .
$$

Using (2-31), apart from the case $\varepsilon=1, \alpha=N=\alpha_{1}$, we can find an $A$ such that $\varepsilon v(\alpha) A^{2}+(N+v(\alpha)) A+\varepsilon \alpha=0$, and $A+\varepsilon \neq 0$. Moreover $\Psi(\bar{Y}) \geq 0$ on $L \cap \mathscr{Q}_{1}$; indeed, $(p-1) \Psi^{\prime}(t)=\left((\delta \ell)^{p-1}+t\right)^{(2-p) /(p-1)}-t^{(2-p) /(p-1)}$, so $\Psi$ has a minimum on $\left(-(\delta \ell)^{p-1}, \infty\right)$ at 0 , and hence is nonnegative on this interval. Then the orientation of the vector field does not change along $L \cap 2_{1}$; in particular no cycle can exist in $2_{1}$; and similarly no homoclinic trajectory can exist. In the case $\varepsilon=1, \alpha=N=\alpha_{1}, Y \equiv y \in[0, \ell)$ defines the trajectory $\mathscr{T}_{r}$, corresponding to the solutions given by (1-6) with $K>0$, and again no cycle can exist in $2_{1}$ : it would intersect $\mathscr{T}_{r}$. 
Next we prove the nonexistence of cycles on one side of the Hopf bifurcation point:

Theorem 2.19. Assume $\delta<N$ and $\varepsilon(\delta-\alpha)<0<\varepsilon(\delta-N / 2)$. If $\varepsilon\left(\alpha-\alpha^{*}\right) \geq 0$, there exists no cycle or homoclinic orbit in $2_{1}$.

Proof. $M_{\ell}$ is a source or weak source if $\varepsilon=1$, and a sink or weak sink if $\varepsilon=-1$. Suppose there exists a cycle in $2_{1}$. Then any trajectory starting from $M_{\ell}$ at $-\varepsilon \infty$ has a limit cycle in $2_{1}$, which is attracting at $\varepsilon \infty$. Such a cycle is not unstable (if $\varepsilon=1$ ) or not stable (if $\varepsilon=-1$ ); in other words the Floquet integral on the period $[0, \mathscr{P}]$ is nonpositive if $\varepsilon=1$ and nonnegative if $\varepsilon=-1$. From (2-50) we then get

$$
\begin{aligned}
\varepsilon \int_{0}^{\mathscr{P}}\left(\frac{\partial f_{1}}{\partial y}(y, Y)+\frac{\partial f_{2}}{\partial Y}(y, Y)\right) d \tau & \\
& =\int_{0}^{\mathscr{\rho}}\left(|2 \delta-N|-\frac{1}{p-1} Y^{(2-p) /(p-1)}\right) d \tau \leq 0 .
\end{aligned}
$$

Now, from (2-28),

$$
\begin{aligned}
& 0=\delta \int_{0}^{\mathscr{P}} \bar{y} d \tau-v(\alpha) \int_{0}^{\mathscr{P}} \bar{Y} d \tau-\int_{0}^{\mathscr{P}} \Psi(\bar{Y}) d \tau \\
& 0=\alpha \int_{0}^{\mathscr{P}} \bar{y} d \tau+(\delta-N-v(\alpha)) \int_{0}^{\mathscr{P}} \bar{Y} d \tau-\int_{0}^{\mathscr{P}} \Psi(\bar{Y}) d \tau .
\end{aligned}
$$

Moreover, since $\Psi$ is nonnegative,

$$
\int_{0}^{\mathscr{P}} \Psi(\bar{Y}) d \tau=-p^{\prime} \int_{0}^{\mathscr{P}} \bar{y} d \tau=-\frac{p^{\prime}(N-\delta)}{\alpha-\delta} \int_{0}^{\mathscr{P}} \bar{Y} d \tau>0 ;
$$

and since $y^{\prime}=\delta y-Y^{1 /(p-1)}$,

$$
\int_{0}^{\mathscr{P}} Y^{1 /(p-1)} d t=\delta \int_{0}^{\mathscr{P}} y d t<\delta \ell \mathscr{P} .
$$

From this, (2-51), and Jensen's inequality, it follows that

$$
\begin{aligned}
(p-1)|2 \delta-N| & \leq \int_{0}^{\mathscr{P}} Y^{(2-p) /(p-1)} d \tau \\
& \leq \mathscr{P}^{p-1}\left(\int_{0}^{\mathscr{P}} Y^{1 /(p-1)} d \tau\right)^{2-p}<(\delta \ell)^{2-p}=\frac{\varepsilon \delta(N-\delta)}{\alpha-\delta} .
\end{aligned}
$$

Hence $\varepsilon\left(\alpha-\alpha^{*}\right)<0$, a contradiction. Next, suppose that there is an homoclinic orbit. From [Hubbard and West 1995, Theorem 9.3, p. 303] we see that the saddle connection is repelling if $\varepsilon=1$ and attracting if $\varepsilon=-1$, because the sum of the eigenvalues $\mu_{1}, \mu_{2}$ of the linearized problem at $(0,0)$ is $2 \delta-N$. That means that the solutions just inside it spiral toward the loop near $-\varepsilon \infty$. Because $M_{\ell}$ is a 
source or weak source or sink or weak sink, such solutions have a limit cycle that is attracting at $\varepsilon \infty$. As before, we reach a contradiction.

Finally we get the nonexistence of cycles in nonobvious cases, where we have shown that any solution has at most one or two zeros.

Theorem 2.20. Assume $\delta<N$ and $\varepsilon(\delta-\alpha)<0<\varepsilon(\delta-N / 2)$. If $\varepsilon=1$ and $\alpha \leq \eta$, or $\varepsilon=-1$ and $-p^{\prime} \leq \alpha<0$, there exists no cycle and no homoclinic orbit in $\mathscr{2}_{1}$.

Proof. (i) Suppose there exists a cycle. There are two possibilities:

Suppose $\varepsilon=1$ and $\alpha \leq \eta$. $M_{\ell}$ is a sink since $\alpha<\alpha^{*}$, so any trajectory converging to $M_{\ell}$ at $\infty$ has a limit cycle 0 in $2_{1}$, attracting at $-\infty$. Let $(y, Y)$ describe the orbit $\mathcal{O}$, of period $\mathscr{P}$. Then $\mathcal{O}$ is not stable, so the Floquet integral is nonnegative, and from (2-51),

$$
\int_{0}^{\mathscr{P}}\left(2 \delta-N-\frac{1}{p-1} Y^{(2-p) /(p-1)}\right) d \tau \geq 0 .
$$

Otherwise $y$ is bounded from above and below; thus the function $y_{\alpha}$, defined by (2-3) with $d=\alpha$, satisfies $\lim _{\tau \rightarrow-\infty} y_{\alpha}=0$ and $\lim _{\tau \rightarrow \infty} y_{\alpha}=\infty$; moreover $y_{\alpha}$ has only minimal points, from (2-35), since $\alpha \leq \eta$; thus $y_{\alpha}^{\prime}>0$ on $\mathbb{R}$. From (2-5) and (2-4) with $d=\alpha$,

$$
\frac{y_{\alpha}^{\prime \prime}}{y_{\alpha}^{\prime}}+\eta-2 \alpha+\frac{1}{p-1} Y^{(2-p) /(p-1)}=\frac{\alpha(\eta-\alpha) y_{\alpha}}{y_{\alpha}^{\prime}}=\frac{\alpha(\eta-\alpha) y_{\alpha}}{\alpha y_{\alpha}-Y_{\alpha}^{1 /(p-1)}}>\eta-\alpha .
$$

Upon integration over $[0, \mathscr{P}]$, this implies $\eta-2 \alpha+2 \delta-N>\eta-\alpha$, which is impossible, since $\delta-N+\delta-\alpha<0$.

Alternatively, suppose $\varepsilon=-1$ and $-p^{\prime} \leq \alpha<0$. $M_{\ell}$ is a source since $\alpha^{*}<\alpha$, and any trajectory converging to it at $-\infty$ has a limit cycle $\mathbb{O}^{\prime}$ attracting at $\infty$. Let $(y, Y)$ describe the orbit $O^{\prime}$, of period $\mathscr{P}$. Then $O^{\prime}$ is not unstable, so the Floquet integral is nonpositive, hence

$$
\int_{0}^{\mathscr{P}}\left(2 \delta-N+\frac{1}{p-1} Y^{(2-p) /(p-1)}\right) d \tau \leq 0 .
$$

Moreover $Y$ is bounded from above and below; thus $Y_{\alpha}$, defined by (2-3) with $d=$ $\alpha$, satisfies $\lim _{\tau \rightarrow-\infty} Y_{\alpha}=\infty, \lim _{\tau \rightarrow \infty} Y_{\alpha}=0$. And $Y_{\alpha}$ has only minimal points, by (2-36), since $-p^{\prime} \leq \alpha<0$; thus $Y_{\alpha}^{\prime}<0$ on $\mathbb{R}$. From (2-6) and (2-4) we get

$$
\begin{aligned}
\frac{Y_{\alpha}^{\prime \prime}}{Y_{\alpha}^{\prime}}+(p-1)\left(\eta-2 \alpha-p^{\prime}\right)-\frac{1}{p-1} Y^{(2-p) /(p-1)} & =\frac{(p-1)^{2}(\eta-\alpha)\left(p^{\prime}+\alpha\right) Y_{\alpha}}{Y_{\alpha}^{\prime}} \\
& <-(p-1)\left(p^{\prime}+\alpha\right) .
\end{aligned}
$$

Upon integration over $[0, \mathscr{P}]$, this implies $(p-1)\left(\eta-2 \alpha-p^{\prime}\right)+2 \delta-N<-(p-1) \times$ $\left(p^{\prime}+\alpha\right)$, which means $p \delta+(p-1)|\alpha|<0$; but this is false. 
(ii) Suppose there exists an homoclinic orbit. Since $\delta<N$, the origin is a saddle point, so $\mathscr{T}_{r}$ is the only trajectory starting from $(0,0)$ in $\mathscr{2}_{1}$, and there exists a unique trajectory $\mathscr{T}_{s}$ converging to $(0,0)$, lying in $\mathscr{Q}_{1}$ for large $\tau$, having infinite slope at $(0,0)$, and satisfying $\lim _{r \rightarrow 0} r^{\eta} w=c>0$.

If $\varepsilon=1$, then $\mathscr{T}_{r}$ satisfies $\lim _{\tau \rightarrow-\infty} e^{-\alpha \tau} y_{\alpha}=a>0$, so $\lim _{\tau \rightarrow-\infty} y_{\alpha}=0$; also $y_{\alpha}$ has only minimal points, so it is increasing and positive; and $\mathscr{T}_{s}$ satisfies $\lim _{\tau \rightarrow \infty} e^{(\eta-\alpha) \tau} y_{\alpha}=c>0$. If $\alpha<\eta$, then $\lim _{\tau \rightarrow \infty} y_{\alpha}=0$, thus $\mathscr{T}_{r} \neq \mathscr{T}_{s}$. If $\alpha=\eta$, $\mathscr{T}_{s}$ is given explicitly by (1-7), that means $y_{\alpha}$ is constant, thus again $\mathscr{T}_{r} \neq \mathscr{T}_{s}$.

If $\varepsilon=-1$, then $\mathscr{T}_{s}$ satisfies $\lim _{\tau \rightarrow-\infty} e^{(\eta-\alpha)(p-1) \tau} Y_{\alpha}>0$, because $\lim _{\tau \rightarrow-\infty} \zeta=$ $\eta$; so $\lim _{\tau \rightarrow \infty} Y_{\alpha}=0$. Moreover $Y_{\alpha}$ has only minimal points, and hence is increasing and positive; otherwise $\mathscr{T}_{r}$ satisfies $\lim _{\tau \rightarrow-\infty} e^{-(\alpha(p-1)+p) \tau} Y_{\alpha}=-a \alpha / N>0$, by (2-33). If $\alpha>-p^{\prime}$, we get $\lim _{\tau \rightarrow \infty} Y_{\alpha}=0$, which implies $\mathscr{T}_{r} \neq \mathscr{T}_{s}$. If $\alpha=-p^{\prime}$, then $\mathscr{T}_{r}$ is given explicitly by $(1-8)$; in other words $Y_{\alpha}$ is constant, and again $\mathscr{T}_{r} \neq \mathscr{T}_{s}$.

Boundedness of cycles. When there do exist cycles, except for a few cases, we cannot prove their uniqueness, but we can show:

Theorem 2.21. When nonempty, the set $\mathscr{C}$ of cycles of system $(S)$ is bounded in $\mathbb{R}^{2}$. Proof. Suppose there exists a cycle $\mathbb{O}$ in $\mathbb{R}^{2}$. By Propositions 2.5, 2.7, 2.10, 2.11 and Theorem 2.20, this can happen only in four cases: $\varepsilon=1, N<\alpha<\delta ; \varepsilon=1, N<$ $\delta=\alpha ; \varepsilon=1, \max (\delta, N, \eta)<\alpha, N / 2<\delta ; \varepsilon=-1, \delta<N / 2, \alpha<-p^{\prime}$. In the first case, $\mathscr{C}$ is bounded and lies in $(-\ell, \ell) \times\left(-(\delta \ell)^{p-1},(\delta \ell)^{p-1}\right)$, by Proposition 2.10. In the other cases we use the energy function $W$. Let $(y, Y)$ describe the trajectory 0 . Then $W$ is periodic, and its maximum and minimum points are precisely the points of the curve $\mathscr{L}$. Indeed if $W^{\prime}\left(\tau_{1}\right)=0$ and the point $\left(y\left(\tau_{1}\right), Y\left(\tau_{1}\right)\right.$ is not on $\mathscr{L}$, it lies on the curve $M$ defined in $(2-11)$; hence $y^{\prime}\left(\tau_{1}\right)=0$ and $y^{\prime \prime}\left(\tau_{1}\right) \neq 0$, since 0 is not just a stationary point. Therefore $\left.\left(\delta y-|Y|^{(2-p) /(p-1)} Y\right)(|\delta y|)^{p-2} \delta y-Y\right)>0$ near $\tau_{1}$; then $W^{\prime}$ has constant sign, and $\tau_{1}$ is not a maximum or a minimum. In this way we obtain estimates for $W$ independently of the trajectory:

$$
\max _{\tau \in \mathbb{R}}|W(\tau)|=M=\max _{(y, Y) \in \mathscr{L}}|\mathcal{W}(y, Y)| .
$$

At the maximal points $\tau$ of $y$, one has $|Y(\tau)|^{(2-p) /(p-1)} Y(\tau)=\delta y(\tau)$, so

$$
W(\tau)=\frac{\varepsilon(\delta-N) \delta^{p-1}}{p}|y(\tau)|^{p}+\frac{\alpha-\delta}{2} y^{2}(\tau) .
$$

By the Hölder inequality, $y$ is bounded by a constant independent of the trajectory, and

$$
\frac{|Y|^{p^{\prime}}}{p^{\prime}} \leq \delta y Y+\frac{|2 \delta-N| \delta^{p-1}}{p}|y|^{p}+\frac{|\alpha-\delta|}{2} y^{2}+M .
$$

Thus $Y$ is also uniformly bounded, and $\mathscr{C}$ is bounded. 


\section{The case $\varepsilon=1, \alpha<\delta$ or $\alpha=\delta<N$}

Lemma 3.1. Assume $\varepsilon=1$ and $-\infty<\max (\alpha, N)<\delta(\alpha \neq 0)$. In the phase plane $(y, Y)$, there exist

(i) a trajectory $\mathscr{T}_{1}$ converging to $M_{\ell}$ at $\infty$, such that $y$ is increasing as long as it is positive;

(ii) a trajectory $\mathscr{T}_{2}$ in $\mathscr{2}_{1} \cup \mathscr{2}_{4}$ converging to $M_{\ell}$ at $-\infty$, and unbounded at $\infty$, with $\lim _{\tau \rightarrow \infty} \zeta=\alpha$;

(iii) a trajectory $\mathscr{T}_{3}$ converging to $M_{\ell}$ at $-\infty$, such that $y$ has at least one zero;

(iv) a trajectory $\mathcal{T}_{4}$ in $2_{1}$, converging to $M_{\ell}$ at $\infty$, with $\lim _{\tau \rightarrow \ln R_{w}} Y / y=1$;

(v) trajectories $\mathscr{T}_{5}$ in $\mathscr{2}_{1} \cup \mathscr{2}_{4}$ unbounded at $\pm \infty$, with

$$
\lim _{\tau \rightarrow \infty} \zeta=\alpha \quad \text { and } \lim _{\tau \rightarrow \ln R_{w}} Y / y=1 .
$$

Proof. Here the system $(S)$ has three stationary points, defined by (2-26). The point $(0,0)$ is a source, and the point $M_{\ell}$ is a saddle point. The eigenvalues satisfy $\lambda_{1}<0<\lambda_{2}<\delta$. The eigenvectors $u_{1}=\left(-v(\alpha), \lambda_{1}-\delta\right)$ and $u_{2}=\left(v(\alpha), \delta-\lambda_{2}\right)$ form a positively oriented basis, and $u_{1}$ points toward $2_{3}$, while $u_{2}$ points toward $2_{1}$. There exist four particular trajectories converging to $M_{\ell}$ at $\pm \infty$, namely:

- $\mathscr{T}_{1}$ converging to $M_{\ell}$ at $\infty$, with tangent vector $u_{1}$; then $y<\ell$ and $Y<(\delta \ell)^{p-1}$ and $y^{\prime}>0$ near $\infty$; as above, $y$ cannot have a local minimum, so $y^{\prime}>0$ whenever $y>0$.

- $\mathscr{T}_{2}$ converging to $M_{\ell}$ at $-\infty$, with tangent vector $u_{2}$; then $y^{\prime}>0$ near $-\infty$. If $y$ has a local maximum at some $\tau$, then $y^{\prime \prime}(\tau) \leq 0$, so that $y(\tau) \leq \ell$ from (2-16), which is impossible. Then $y$ is increasing on $\mathbb{R}$ and $\lim _{\tau \rightarrow \infty} y=\infty$, and $\lim _{\tau \rightarrow \infty} \zeta=\alpha$ from Proposition 2.8. In particular $\mathscr{T}_{2}$ stays in $2_{1}$ if $\alpha>0$, and enters $2_{4}$ if $\alpha<0$.

- $\mathscr{T}_{3}$ converging to $M_{\ell}$ at $-\infty$, with tangent vector $-u_{2}$; then $y^{\prime}<0$ near $-\infty$. If $y$ has a local minimum at some $\tau$, then $y(\tau) \geq \ell$, which is still impossible. Thus $y$ is decreasing at long as the trajectory stays in $\mathscr{2}_{1}$. It cannot stay in it, because it cannot converge to $(0,0)$. It cannot enter $2_{4}$ by Remark 2.1(i) on page 211. Then it enters $2_{2}$ and $y$ has at least one zero.

- $\mathscr{T}_{4}$ converging to $M_{\ell}$ at $\infty$, with tangent vector $-u_{1}$; then $y^{\prime}<0$ near $\infty$. As above, $y$ cannot have a local maximum, it is decreasing and $\lim _{\tau \rightarrow \ln R_{w}} y=$ $\infty$. From Proposition 2.8, $y$ cannot be defined near $-\infty$, hence $R_{w}>0$ and $\lim _{\tau \rightarrow \ln R_{w}} Y / y=1$.

For any trajectory $\mathscr{T}$ in the domain delimited by $\mathscr{T}_{2}$ and $\mathscr{T}_{4}$, the function $y$ is positive, and $\mathcal{T}$ cannot converge to $M_{\ell}$ at $\infty$, and $y$ is monotone for large $\tau$ from 
Proposition 2.7, because $\alpha<\delta$; thus $\lim _{\tau \rightarrow \infty} \zeta=\alpha$ from Proposition 2.8, and $y$ is not defined near $-\infty$, and $\mathscr{T}$ is of type (5).

We now study the various global behaviors, according to the values of $\alpha$. The results are expressed in terms of $w$.

$$
\alpha \leq N<\delta
$$

Theorem 3.2. Assume the $\varepsilon=1$ and $-\infty<\alpha \leq N<\delta$, with $\alpha \neq 0$. All regular solutions $w$ of $\left(E_{w}\right)$ have constant sign, and $\lim _{r \rightarrow \infty} r^{\alpha}|w|=L>0$ if $\alpha<N$, $\lim _{r \rightarrow \infty} r^{\delta}|w|=\ell$ if $\alpha=N$. And $w(r)=\ell r^{-\delta}$ is also a solution. There exist solutions satisfying any one of these characterizations:

(1) (only if $\alpha<N$ ) $w$ is positive, $\lim _{r \rightarrow 0} r^{\eta} w=c>0$, if $N \geq 2$ (and (2-41) holds with $a>0>b$ if $N=1$ ), and $\lim _{r \rightarrow \infty} r^{\delta} w=\ell$;

(2) $w$ is positive, $\lim _{r \rightarrow 0} r^{\delta} w=\ell, \lim _{r \rightarrow \infty} r^{\alpha} w=L>0$;

(3) $w$ has precisely one zero, $\lim _{r \rightarrow 0} r^{\delta} w=\ell, \lim _{r \rightarrow \infty} r^{\alpha} w(r)=L<0$;

(4) $w$ is positive, $R_{w}>0, \lim _{r \rightarrow \infty} r^{\delta} w=\ell$;

(5) $w$ is positive, $R_{w}>0, \lim _{r \rightarrow \infty} r^{\alpha} w=L>0$;

(6) $w$ has one zero, $R_{w}>0$, and $\lim _{r \rightarrow \infty} r^{\alpha} w=L \neq 0$;

(7) (only if $\alpha<N$ ) $w$ is positive, $\lim _{r \rightarrow 0} r^{\eta} w=c>0$ if $N \geq 2$ (and (2-41) holds with $a>0>b$ if $N=1$ ), and $\lim _{r \rightarrow \infty} r^{\alpha} w=L>0$;

(8) $w$ has one zero, with $\lim _{r \rightarrow 0} r^{\eta} w=c>0$ if $N \geq 2$ (and (2-41) holds with $a>0>b$ if $N=1$ ), and $\lim _{r \rightarrow \infty} r^{\alpha} w=-L<0$;

(9) $N=1, w>0$ and (2-41) holds with $a \geq 0, b>0$ and $\lim _{r \rightarrow \infty} r^{\alpha} w=L$.

Up to symmetry, all the solutions of $\left(E_{w}\right)$ are as above.
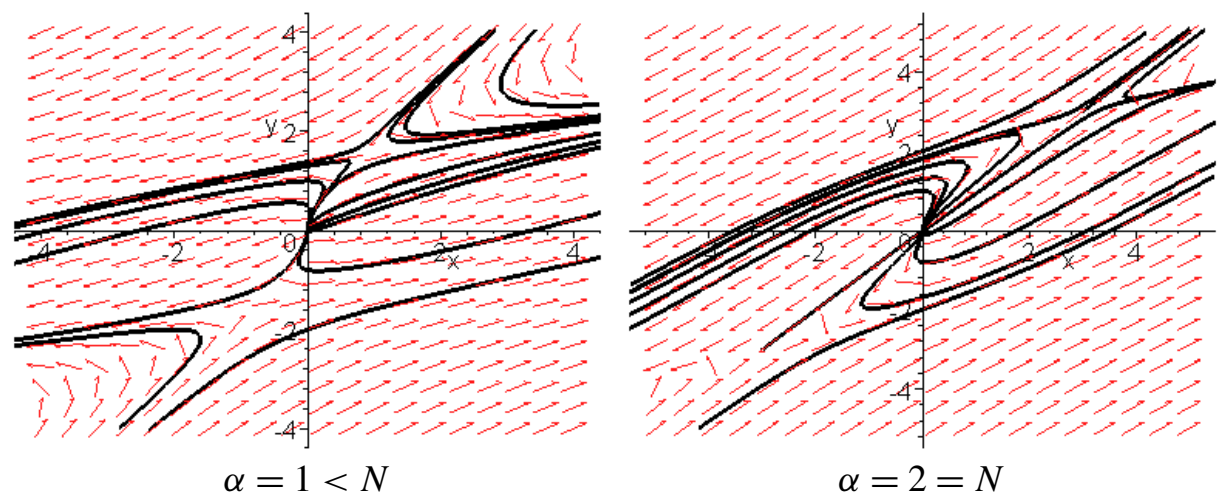

Figure 1. Theorem 3.2: $N=2<\delta=3$. 
Proof. (i) We first assume that $\alpha \neq N$, and refer to Figure 1, left. The trajectory $\mathscr{T}_{r}$ starts in $2_{1}$ for $\alpha>0$, in $2_{4}$ for $\alpha<0$, and $y$ stays positive. Then $\lim _{\tau \rightarrow \infty} y=\infty$, and $\lim _{\tau \rightarrow \infty} \zeta=\alpha$, and $\lim _{r \rightarrow \infty} r^{\alpha} w=L>0$, by Propositions 2.10 and 2.13, since $\alpha<N$. Moreover $y$ is increasing: indeed if it has a local maximum, at this point $y \leq \ell$, and then $y$ has no local minimum, since at such a point $y \geq \ell$, so that $y$ cannot tend to $\infty$. Then $\mathscr{T}_{r}$ stays in $\mathscr{2}_{1}$, and $Y$ is increasing from 0 to $\infty$. Indeed each extremal point $\tau$ of $Y$ is a local minimum, from (2-17). If $\alpha<0$, in the same way, then $Y$ is decreasing from 0 to $-\infty$, and $\mathscr{T}_{r}$ stays in $2_{4}$.

First we follow the trajectory $\mathscr{T}_{1}$ : it does not intersect $\mathscr{T}_{r}$, and cannot enter $\mathscr{2}_{2}$ by Remark 2.1(i). Thus $y$ stays positive and increasing. It cannot enter $2_{4}$, seeing that it does not meet $\mathscr{T}_{r}$ if $\alpha>0$, or (by the same remark) if $\alpha<0$. Thus $\mathscr{T}_{1}$ stays in $2_{1}$, and $(y, Y)$ converges necessarily to $(0,0)$. If $N \geq 2$, then $\lim _{\tau \rightarrow-\infty} \zeta=\eta$, $\lim _{r \rightarrow 0} r^{\eta} w=c>0$ from Proposition 2.8 and 2.9. If $N=1$, since $\mathscr{T}_{1}$ stays in $\mathscr{2}_{1}$, then necessarily $\lim _{\tau \rightarrow-\infty} \zeta=0$, thus (2-41) holds with $a>0>b$.

Next we follow $\mathscr{T}_{3}$ : here $y$ has a zero, which is unique by Proposition 2.5 , since $\alpha<N$. Then $y<0$, and $\lim _{\tau \rightarrow \infty} y=-\infty, \lim _{r \rightarrow \infty} r^{\alpha} w=-L<0$ by Propositions 2.8 and 2.9. $\mathscr{T}_{3}$ stays in $\mathscr{2}_{2}$ if $\alpha<0$, or goes from $\mathscr{2}_{2}$ into $\mathscr{2}_{3}$ if $\alpha>0$.

Trajectories $\mathscr{T}_{2}, \mathscr{T}_{4}, \mathscr{T}_{5}$ of Lemma 3.1 yield solutions $w$ of type (2), (4), (5).

For any trajectories $\mathscr{T}_{6}$ in the domain delimited by $\mathscr{T}_{3}, \mathscr{T}_{4}, y$ has one zero, and $\lim _{r \rightarrow \infty} r^{\alpha} w=L \neq 0$; and $w$ is of type (6).

The solutions of type (7) correspond to the trajectories $\mathscr{T}$ in the domain delimited by $\mathscr{T}_{r}, \mathscr{T}_{1}, \mathscr{T}_{2}$. Indeed $\lim _{\tau \rightarrow \infty} y=\infty$, and $\lim _{r \rightarrow \infty} r^{\alpha} w=L>0$. And $\lim _{\tau \rightarrow-\infty} y=0$. If $N \geq 2$, then $\lim _{\tau \rightarrow-\infty} \zeta=\eta, \lim _{r \rightarrow 0} r^{\eta} w=c>0$, from Proposition 2.8 and 2.9. If $N=1, \mathscr{T}$ cannot meet $\mathscr{T}_{r}$, thus necessarily $\lim _{\tau \rightarrow-\infty} \zeta=0$, and (2-41) holds with $a>0>b$.

Up to a change of $w$ into $-w$, the solutions of type (8) and (9) correspond to the trajectories in the domain delimited by $-\mathscr{T}_{r}, \mathscr{T}_{1}, \mathscr{T}_{3}$. Indeed they satisfy $\lim _{\tau \rightarrow \infty} y=-\infty$, and $\lim _{r \rightarrow \infty} r^{\alpha} w=L<0$; and $\lim _{\tau \rightarrow-\infty} y=0$. If $N \geq 2$, then $\lim _{r \rightarrow 0} r^{\eta} w=c>0$ and $w$ has a zero. If $N=1$, either (2-41) holds with $a=0>b$ and $w$ stays negative, or $a<0, b<0$ and $w$ has a zero. Such solutions exist from Theorem 2.2. By symmetry, all the solutions are described.

(ii) Now assume $\alpha=N$ (Figure 1, right). Then $M_{\ell}$ belongs to the line $y=Y$, and

$$
u_{1}=(-\delta /(p-1),-\delta /(p-1))
$$

has the same direction. Moreover $J_{N}$ is constant, which means $y-Y=C e^{(\delta-N) \tau}$, with $C \in \mathbb{R}$. The solutions corresponding to $C=0$ satisfy $y \equiv Y$, thus $\mathscr{T}_{1}=$ $\mathscr{T}_{r}=\{(\xi, \xi): \xi \in[0, \ell)\}$, corresponding to the regular Barenblatt solutions. And $\mathscr{T}_{4}=\{(\xi, \xi): \xi>\ell\}$ yields the solutions defined by (1-6) for $K<0$. All other solutions exist as before, apart from type (7). 
Note. The trajectory $\mathscr{T}_{1}$ is the only one joining the stationary points $(0,0)$ and $M_{\ell}$. Hence, for $\alpha<N$, solutions $w$ of type (1) are unique, up to the scaling mentioned in the note on page 210. Solutions of types (2), (4), and (5) are also unique.

$$
N<\alpha<\delta
$$

Here we prove that some periodic trajectories can exist, according to the value of $\alpha$ with respect to $\alpha^{*}$. By (2-32), $N<\alpha^{*}$ whenever $\delta^{2}-(N+3) \delta+N>0$, and in particular for any $p \leq \frac{3}{2}$. Our main tool is the Poincaré-Bendixson theorem, using the level curves of the energy function $\mathcal{W}$ :

Lemma 3.3. Assume $\varepsilon=1$ and $N<\alpha<\delta$. Consider, for $k \in \mathbb{R}$, the level curves

$$
\mathscr{C}_{k}=\left\{(y, Y) \in \mathbb{R}^{2}: \mathscr{W}(y, Y)=k\right\}
$$

of the function $\mathcal{W}$ defined in (2-21). They are symmetric with respect to $(0,0)$. Let

$$
k_{\ell}=\mathcal{W}\left(\ell,(\delta \ell)^{p-1}\right)=\frac{1}{2}(\delta-N) \delta^{p-2} \ell^{p} .
$$

If $k>k_{\ell}$, then $\mathscr{C}_{k}$ has two unbounded connected components. If $0<k<k_{\ell}, \mathscr{C}_{k}$ has three connected components, of which one is bounded. If $k=k_{\ell}, \mathscr{C}_{k_{\ell}}$ is connected with a double point at $M_{\ell}$. If $k=0$, one of the three connected components of $\mathscr{C}_{0}$ is $\{(0,0)\}$. If $k<0, \mathscr{C}_{k}$ has two unbounded connected components.

Proof. The energy $k_{\ell}$ of the statement is positive. Also $(y, Y) \in C_{k}$ if and only if $F(y)=k-G(y)$, where $F, G$ are defined in (2-45). By symmetry we can reduce the study of $C_{k}$ to the set $y>0$. Let $\varphi(s)=|s|^{p^{\prime}} / p^{\prime}-s+1 / p$ for any $s \in \mathbb{R}$, and set $\theta=Y /(\delta y)^{p-1}$. Then (2-44) reduces to

$$
\varphi(\theta)=(k-G(y)) /(\delta y)^{p} .
$$

The function $\varphi$ is decreasing on $(-\infty, 1)$ from $\infty$ to 0 , and increasing on $(1, \infty)$ from 0 to $\infty$. Let $\psi_{1}$ be the inverse of the restriction of $\varphi$ to $(-\infty, 1]$ and $\psi_{2}$ the inverse of the restriction of $\varphi$ to $[1, \infty)$, both defined on $[0, \infty)$. For any $y>0$,

$$
y \in \mathscr{C}_{k} \Longleftrightarrow Y=\Phi_{1}(y)<(\delta y)^{p-1} \text { or } Y=\Phi_{2}(y) \geq(\delta y)^{p-1},
$$

where

$$
\Phi_{i}(y)=(\delta y)^{p-1} \psi_{i}\left(\frac{k-G(y)}{(\delta y)^{p}}\right) \quad \text { for } i=1,2,
$$

$\Phi_{1}$ lies below $\mathcal{M}$ whereas $\Phi_{2}$ lies above $\mathcal{M}$, and $\Phi_{1}, \Phi_{2} \in C^{1}((0, \infty))$. The function $G$ has a maximal point at $y=\ell$, and $G(\ell)=k_{\ell}$. Using symmetry we see that either $k>k_{\ell}$ and $y$ ranges over $\mathbb{R}$, in which case $\mathscr{C}_{k}$ has two unbounded connected components; or $0<k<k_{\ell}$ and $\mathscr{C}_{k}$ has three connected components, one of which, $\mathscr{C}_{k}^{b}$, is bounded; or $k=k_{\ell}$ and $\mathscr{C}_{k_{\ell}}$ is connected with a double point at $M_{\ell}$; or yet $k=0$ and one of the three connected components of $\mathscr{C}_{0}$ is $\{(0,0)\}$; or $k<0$ and $\mathscr{C}_{k}$ 
has two unbounded connected components. The unbounded components satisfy $\lim _{|y| \rightarrow \infty} Y / y^{2 / p^{\prime}}= \pm\left(p^{\prime}(\delta \alpha) / 2\right)^{1 / p^{\prime}}$, by (3-1). The zeros of $\Phi_{i}^{\prime}$ are contained in

$$
\mathcal{N}=\left\{(y, Y) \in \mathbb{R}^{2}: y>0, \delta Y=-(\delta-\alpha) y+(2 \delta-N)(\delta y)^{p-1}\right\},
$$

and $\mathcal{N}$ lies above $M$ as long as $y<\ell$.

We now describe $\mathscr{C}_{k}^{b}$ when $0<k \leq k_{\ell}$. The function $\Phi_{1}$ is increasing on a segment $[0, \bar{y}]$ such that $\bar{y}<\ell$, and $\Phi_{1}(0)=-\left(k p^{\prime}\right)^{1 / p^{\prime}}$ and $\left(\bar{y}, \Phi_{1}(\bar{y})\right) \in \mathcal{M}$, with an infinite slope at this point; $\Phi_{2}$ is increasing on some interval $[0, \tilde{y})$ such that $\left(\tilde{y}, \Phi_{2}(\tilde{y})\right) \in \mathcal{N}$ and then decreasing on $(\tilde{y}, \bar{y}]$, and $\Phi_{2}(0)=\left(k p^{\prime}\right)^{1 / p^{\prime}}$ and $\Phi_{2}(\bar{y})=$ $\Phi_{1}(\bar{y})$. By symmetry with respect to $(0,0)$, the curve $\mathscr{C}_{k}^{b}$ is completely described.

Next consider $\mathscr{C}_{k_{\ell}}$ for $y>0$ : the function $\Phi_{2}$ is increasing on $[0, \infty)$ from $\left(p^{\prime} k_{\ell}\right)^{1 / p^{\prime}}$ to $\infty$, and $\Phi_{2}(\ell)=(\delta \ell)^{p-1}$; the function $\Phi_{1}$ is increasing on some interval $[0, \hat{y})$ such that $\left(\hat{y}, \Phi_{1}(\hat{y})\right) \in \mathcal{N}$, so $\hat{y}>\ell$; and $\left(\hat{y}, \Phi_{1}(\hat{y})\right)$ is below $M$, and $\Phi_{1}(\ell)=(\delta \ell)^{p-1}$, and $\Phi_{1}$ is decreasing on $(\hat{y}, \infty)$, with $\lim _{y \rightarrow \infty} \Phi_{1}=-\infty$. Setting $\mathscr{C}_{k_{\ell}, 1}=\left\{\left(y, \Phi_{1}(y)\right): y>\ell\right\}$ and $\mathscr{C}_{k_{\ell}, 2}=\left\{\left(y, \Phi_{2}(y)\right): y>\ell\right\}$, one has $\mathscr{C}_{k_{\ell}}=\mathscr{C}_{k_{\ell}}^{b} \cup \pm \mathscr{C}_{k_{\ell}, 1} \cup \mathscr{C}_{k_{\ell}, 2}$.

Theorem 3.4. Assume $\varepsilon=1$ and $N<\alpha<\delta$. Then $w(r)=\ell r^{-\delta}$ is a solution of $\left(E_{w}\right)$.

(i) If $\alpha \leq \alpha^{*}$, any solution of $\left(E_{w}\right)$ has at most a finite number of zeros.

(ii) There exist $\check{\alpha}$ such that $\max \left(N, \alpha^{*}\right)<\check{\alpha}<\delta$, such that if $\alpha>\check{\alpha}$, in the phase plane $(y, Y)$, there exists a cycle surrounding $(0,0)$.

(iii) Let $\alpha$ be such that there exists no such cycle. Then all regular solutions have a finite positive number of zeros and $\lim _{r \rightarrow \infty} r^{\alpha} w=L_{r} \neq 0$ or $\lim _{r \rightarrow \infty} r^{\delta} w=$ $\pm \ell$. There exist solutions of types (2)-(6) of Theorem 3.2, and solutions such that

(1') (only if $L_{r} \neq 0$ ) $\lim _{r \rightarrow 0} r^{\delta} w=\ell$, and $\lim _{r \rightarrow 0} r^{\eta} w=c \neq 0$ (or (2-41) holds if $N=1)$;

(7') $\lim _{r \rightarrow 0} r^{\eta} w=c \neq 0$ (or (2-41) holds if $\left.N=1\right)$ and $\lim _{r \rightarrow \infty} r^{\alpha} w=L \neq 0$.

(iv) For any $\alpha$ such that there exists such a cycle, there exist solutions $w$ which oscillate near 0 and $\infty$, and $r^{\delta} w$ is periodic in $\ln r$. All regular solutions $w$ oscillate near $\infty$, and $r^{\delta} w$ is asymptotically periodic in $\ln r$. There exist solutions of types (2), (4), (5), and solutions

$\left(1^{\prime \prime}\right)$ with precisely one zero, $R_{w}>0$, and $\lim _{r \rightarrow \infty} r^{\delta} w=\ell$;

$\left(3^{\prime \prime}\right)$ such that $\lim _{r \rightarrow 0} r^{\delta} w=\ell$, and oscillating near $\infty$;

(9) such that $\lim _{r \rightarrow 0} r^{\eta} w=c \neq 0$ (or (2-41) holds if $\left.N=1\right)$ and oscillating near $\infty$;

(10) with precisely one zero, $R_{w}>0$, and $\lim _{r \rightarrow \infty} r^{\alpha} w=L \neq 0$;

(11) with $R_{w}>0$ and oscillating near $\infty$. 
Proof. There always exist solutions of type (2), (4), and (5), by Lemma 3.1.

(i) Assume $\alpha \leq \alpha^{*}$ (see Figure 2, left). Consider any trajectory $\mathscr{T}$. Suppose $y$ has infinitely many zeros near $\pm \infty$. From Proposition $2.10, \mathcal{T}$ is contained in the set

$$
\mathscr{D}=\left\{(y, Y) \in \mathbb{R}^{2}:|y|<\ell,|Y|<(\delta \ell)^{p-1}\right\}
$$

near $\pm \infty$. Then $\mathscr{T}$ is bounded near $\pm \infty$, hence the limit set at $\pm \infty$ is contained in $\mathscr{D}$. But $M_{\ell} \notin \mathscr{D}$, and $(0,0)$ is a source and a node point, so it cannot be in the limit set $\Gamma$ at $\infty$. From the Poincaré-Bendixson theorem, $\Gamma$ is a closed orbit, so that there exists a cycle. Moreover, from (2-25), (2-49) and (2-50),

$$
\frac{\partial f_{1}}{\partial y}(y, Y)+\frac{\partial f_{2}}{\partial Y}(y, Y)=\frac{1}{p-1}\left(D^{(2-p) /(p-1)}-|Y|^{(2-p) /(p-1)}\right)
$$

thus, by Bendixson's criterion, the set $\{|Y|<D\}$ contains no cycle. Now note that

$$
\alpha \leq \alpha^{*} \Longleftrightarrow(\delta \ell)^{p-1} \leq D
$$

Then there is no cycle in $\mathscr{D}$, and we reach a contradiction.

(ii) Now assume $\alpha>\max \left(N, \alpha^{*}\right)$. The curve $\mathscr{L}$ intersects $\mathcal{M}$ at $\left(\delta^{-1} D^{1 /(p-1)}, D\right)$. Then

$$
\mathscr{S}_{\mathscr{L}} \cap \mathcal{M}=\left\{\left(\delta^{-1}(\theta D)^{1 /(p-1)}, \theta D\right): \theta \in[0,1]\right\} ;
$$

and $D<(\delta \ell)^{p-1}$ by (3-2), so $\mathscr{S}_{\mathscr{L}}$ does not contain $M_{\ell}$. We can find $k_{1}>0$ small enough that $\mathscr{C}_{k_{1}}^{b}$ is interior to $\mathscr{Y}_{\mathscr{L}}$. Next we search for $k \in\left(0, k_{\ell}\right)$ such that $\mathscr{L}$ is in the domain delimited by $C_{k}^{b}$. By symmetry we only consider the points of $\mathscr{L}$ such that $y \geq 0$. In any case for any point of $\mathscr{L}$ we have $|\delta y|^{p}+|Y|^{p^{\prime}} \leq M=(2(2 \delta-N))^{\delta}$, by (2-23) and by convexity. By a straightforward computation this implies that

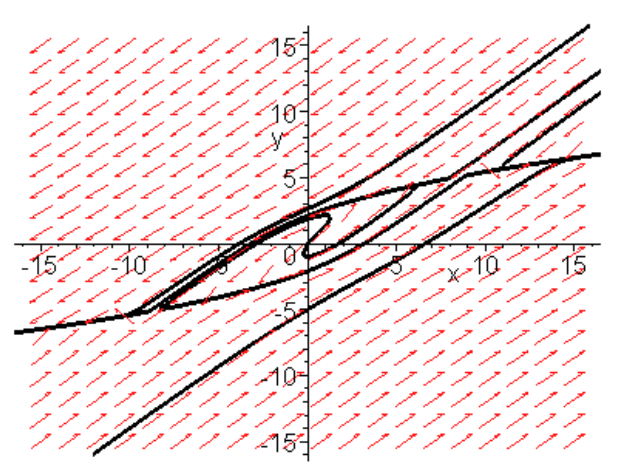

$\alpha=2.41$

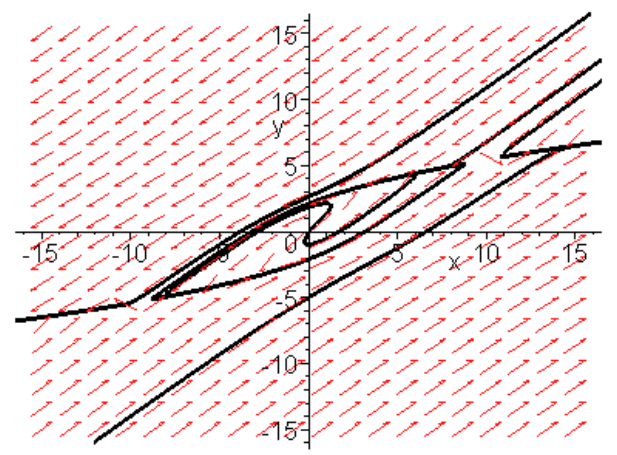

$\alpha=2.42$

Figure 2. Theorem 3.4: $\varepsilon=1, N=2<\alpha<\delta=3$. 
$\mathscr{W}(y, Y) \leq K M$, where $K=\max \left(2 / p^{\prime},(3 \delta-N) / \delta p\right)$. Let $\check{\alpha}=\check{\alpha}(\delta, N)$ be given by $K M=k_{\ell}$. This means that

$$
\delta-\check{\alpha}=\left(\frac{\delta-N}{2 K \delta^{2-p}}\right)^{1 / \delta} \frac{\delta^{p-1}(\delta-N)}{2(2 \delta-N)} .
$$

If $\alpha>\check{\alpha}$, there exists $k_{2}<k_{\ell}$ such that $\mathscr{L}$ is contained in the set

$$
\left\{(y, Y) \in \mathbb{R}^{2}: \mathcal{W}(y, Y)<k_{2}\right\},
$$

which has three connected components; because $\mathscr{S}_{\mathscr{L}}$ is connected, it is contained in the interior to $\mathscr{C}_{k_{2}^{b}}$. Then the domain delimited by $\mathscr{C}_{k_{1}}^{b}$ and $\mathscr{C}_{k_{2}}^{b}$ is bounded and forward invariant. It does not contain any stationary point, and so it contains a cycle, by the Poincaré-Bendixson theorem (see Figure 2, right).

(iii) Let $\alpha$ be such that there exists no cycle. Since $N<\alpha$, all regular solutions $y$ have at least one zero. They have a finite number of zeros. For if not, $(y, Y)$ is bounded near $\infty$, so it has a limit cycle. Then either $\lim _{\tau \rightarrow \infty} y= \pm \infty$ and $\lim _{\tau \rightarrow \infty} \zeta=\alpha>0$, so that the trajectory $\mathscr{T}_{r}$ ends up in $2_{1} \cup 2_{3}$ and $\lim r^{\alpha} w=L_{r} \neq 0$, or else $\lim _{\tau \rightarrow \infty} y= \pm \ell$ and $\lim _{r \rightarrow \infty} r^{\delta} w= \pm \ell$.

$\mathscr{T}_{3}$ cannot meet $\mathscr{T}_{r}$ or $-\mathscr{T}_{r}$, thus $y$ has a unique zero, and $\lim _{\tau \rightarrow \infty} y=-\infty$, and $\lim _{\tau \rightarrow \infty} \zeta=\alpha$. The same happens for the trajectories $\mathscr{T}_{6}$ in the domain delimited by $\mathscr{T}_{3}, \mathscr{T}_{4}$. Thus there exist solutions of types (3) and (6).

Suppose $L_{r} \neq 0$ and consider $\mathscr{T}_{1}$ : the trajectories $\mathscr{T}_{r},-\mathscr{T}_{r}, \mathscr{T}_{1}$ have a last intersection point at time $\tau_{0}$ with the half-axis $\{y=0, Y<0\}$ at some points $P_{r}, P_{r}^{\prime}, P_{1}$, and $P_{1} \in\left[P_{r}, P_{r}^{\prime}\right]$. The domain delimited by $\mathscr{T}_{r},-\mathscr{T}_{r}$ and $\left[P_{r}, P_{r}^{\prime}\right]$ is bounded and backward invariant, by Remark 2.1(i) on page 211. Then $\mathscr{T}_{1}$ stays in it for $\tau<\tau_{0}$, it has a finite number of zeros, and converges to $(0,0)$ near $-\infty$; thus $w$ is of type $\left(1^{\prime}\right)$. If $N \geq 2$, then $\lim _{\tau \rightarrow \infty} \zeta=\eta$, so that $y$ has at least one zero.

Since $(0,0)$ is a source, there exist other solutions converging to $(0,0)$ near $-\infty$, they have a finite number of zeros, and $\lim _{\tau \rightarrow \infty} \zeta=\alpha$, and $w$ is of type $\left(7^{\prime}\right)$. (iv) Let $\alpha$ such that there exists a cycle, thus $\mathscr{T}_{r}$ has a limit cycle $\mathcal{O}$.

Consider again $\mathscr{T}_{1}$. Since $M_{\ell} \notin \mathscr{Y}_{\mathscr{L}}$, the function $W$ is decreasing near $\infty$, so that $W(\tau)>k_{\ell}$; thus $\mathscr{T}_{1}$ is exterior to $\mathscr{C}_{k_{\ell}}^{b}$ for large $\tau$, in the domain exterior to $\mathscr{C}_{k_{\ell}}^{b}$ delimited by $\mathscr{C}_{k_{\ell}, 1}$ and $-\mathscr{C}_{k_{\ell}, 2}$; and it cannot cut $\mathscr{C}_{k_{\ell}}$. Moreover $y$ is decreasing at long as $y>0$, then $\mathscr{T}_{1}$ enters $\mathscr{2}_{4}$ as $\tau$ decreases. It cannot stay in it, because it would converge to $(0,0)$, which is impossible. Then $y$ has at least one zero, and $\mathscr{T}_{1}$ enters $2_{3}$. It stays in it, since it cannot cross $-\mathscr{C}_{k_{\ell}, 2}$. Thus $y$ has a unique zero, and $\lim _{\tau \rightarrow-\infty} y=-\infty$, and $R_{w}>0$ from Proposition 2.8, because $\mathscr{T}_{1}$ cannot converge to $(0,0)$ at $-\infty$, and $w$ is of type $\left(1^{\prime \prime}\right)$.

Next consider $\mathscr{T}_{3}$. Here $W$ is decreasing near $-\infty$, hence $W(\tau)<k_{\ell}$; thus $\mathscr{T}_{3}$ is in the interior of $\mathscr{C}_{k_{\ell}}^{b}$ near $-\infty$. Now the domain delimited by $\mathscr{C}_{k_{1}}^{b}$ and $\mathscr{C}_{k_{\ell}}^{b}$ is 
forward invariant, thus $\mathscr{T}_{3}$ stays in it; then it is bounded, and has a limit cycle at $\infty$, and $w$ is of type $\left(3^{\prime \prime}\right)$.

The solutions of type (9) correspond to trajectories $\mathscr{T}$ in the domain delimited by $\mathcal{O}$, and distinct from $\mathcal{T}_{r}$. Indeed $\mathcal{T}$ is bounded, in particular the limit-set at $-\infty$ is $(0,0)$, or a closed orbit. But $\mathscr{T}$ cannot intersect $\mathscr{T}_{r}$. Then $\mathscr{T}$ converges to $(0,0)$ near $-\infty$.

The solutions of type (10) correspond to a trajectory $\mathscr{T}$ in the domain delimited by $\mathscr{T}_{1} \cup \mathscr{T}_{2}$ (or its opposite): indeed $y$ has constant sign near $\infty$ and near $\ln R_{w}$, and $\lim _{r \rightarrow \infty} r^{\alpha} w=L \neq 0$, and $R_{w}>0$, from Proposition 2.8. Then $\mathcal{T}$ starts in $2_{3}$, and ends up in $2_{1}$; and $y$ has at most one zero, because at such a point $y^{\prime}=-|Y|^{1 /(p-1)} Y>0$, thus it has precisely one zero.

Solutions of type (11) correspond to a trajectory $\mathscr{T}$ in the domain delimited by $\mathscr{T}_{1}, \mathscr{T}_{4},-\mathscr{T}_{1},-\mathscr{T}_{4}$. Then $y$ cannot have constant sign near $\infty$ : indeed this implies $\lim \zeta=\alpha>0$; this is impossible since the line $Y=y$ is an asymptotic direction for $\mathscr{T}_{1}, \mathscr{T}_{4}$. Thus $\mathscr{T}$ is bounded near $\infty$, and it has a limit cycle at $\infty$. Near $-\infty, y$ a constant sign, because $\mathscr{T}$ cannot meet $\mathscr{T}_{3}$; and $R_{w}>0$ from Proposition 2.8, and $\mathscr{T}$ has the same asymptotic direction $Y=y$ as $\mathscr{T}_{1}, \mathscr{T}_{4}$.

Note. From numerical studies, we conjecture that $\check{\alpha}$ is unique, and the number of zeros of $w$ increases with $\alpha$ in the range $(N, \check{\alpha})$; and moreover there exists $\alpha_{1}=N<\alpha_{2}<\cdots<\alpha_{n}<\alpha_{n+1}<\ldots$, such that regular solutions have $n$ zeros for any $\alpha \in\left(\alpha_{n}, \alpha_{n+1}\right)$, with $\lim _{r \rightarrow \infty} r^{\alpha} w=L_{r} \neq 0$, and $n+1$ zeros for $\alpha=\alpha_{n+1}$, with $\lim _{r \rightarrow \infty} r^{\delta} w= \pm \ell$.

$$
\alpha \leq \delta \leq N, \alpha \neq N
$$

Here $(0,0)$ is the only stationary point, and $N \geq 2$.

Theorem 3.5. Assume $\varepsilon=1$ and $-\infty<\alpha \leq \delta \leq N, \alpha \neq 0, N$. Then all regular solutions of $\left(E_{w}\right)$ have constant sign, and the positive ones satisfy $\lim _{r \rightarrow \infty} r^{\alpha} w(r)=$ $L>0$ if $\alpha \neq \delta$, or (2-39) holds if $\alpha=\delta$. All the other solutions have a reduced domain $\left(R_{w}>0\right)$. Among them, there exist solutions satisfying any one of these characterizations:

(1) $w$ is positive, $\lim _{r \rightarrow \infty} r^{\eta} w=c \neq 0$ if $\delta<N$, or $\lim _{r \rightarrow \infty} r^{N}(\ln r)^{(N+1) / 2} w=\varrho$ defined in (2-40) if $\delta=N$;

(2) $w$ is positive, $\lim _{r \rightarrow \infty} r^{\alpha} w=L>0$ if $\alpha \neq \delta$, or (2-39) holds if $\alpha=\delta$;

(3) $w$ has one zero, and $\lim _{r \rightarrow \infty} r^{\alpha} w=L \neq 0$ if $\alpha \neq \delta$, or (2-39) holds if $\alpha=\delta$.

Up to symmetry, all the solutions are as above.

Proof. Any solution has at most one zero, by Proposition 2.5. The trajectory $\mathscr{T}_{r}$ starts in $2_{4}$ if $\alpha<0$ (Figure 3, left) and in $2_{1}$ if $\alpha>0$ (Figure 3, right). Moreover $y$ 


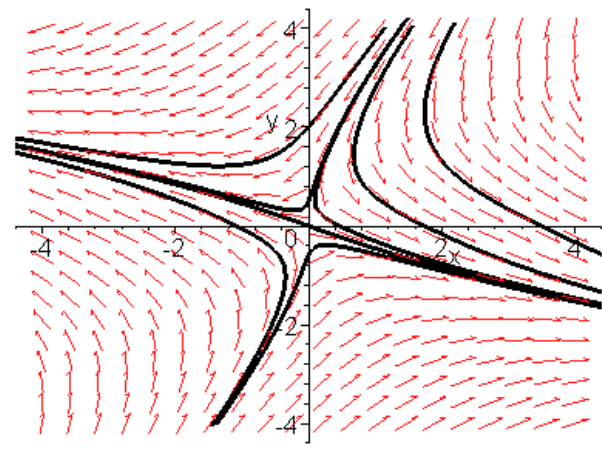

$\alpha=-2$

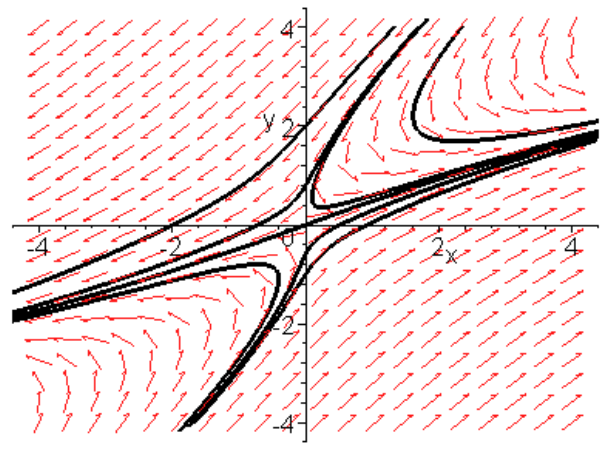

$\alpha=2$

Figure 3. Theorem 3.5: $\varepsilon=1, \alpha<\delta=3<N=4$.

stays positive, and $\lim _{\tau \rightarrow \infty} y=\infty$ and $\lim _{\tau \rightarrow \infty} \zeta=\alpha$, by Proposition 2.13. Then $\lim _{r \rightarrow \infty} r^{\alpha} w(r)=L>0$ if $\alpha<\delta$, or (2-39) holds if $\alpha=\delta$, from Proposition 2.9. Moreover $y$ is increasing: indeed it has no local maximum from (2-16). Thus $\mathscr{T}_{r}$ does not meet $\mathcal{M}$, and so stays below $\mathcal{M}$. If $\alpha>0$, then $\mathscr{T}_{r}$ stays in $\mathscr{2}_{1}$, and $Y$ is increasing from 0 to $\infty$; indeed each extremal point $\tau$ of $Y$ is a local minimum, by (2-17). Likewise, if $\alpha<0$ the function $Y$ is decreasing from 0 to $-\infty$, and $\mathscr{T}_{r}$ stays in $2_{4}$. The only solutions $y$ defined on $(0, \infty)$ are the regular ones, by Proposition 2.8.

For any point $P=\left(\varphi,(\delta \varphi)^{p-1}\right) \in \mathbb{R}^{2}$ with $\varphi>0$, in other words on the curve $\mathcal{M}$, the trajectory $\mathscr{T}_{[P]}$ intersects $\mathcal{M}$ transversally: the vector field is $(0,-(N-\alpha) \varphi)$. Moreover the solution going through this point at time $\tau_{0}$ satisfies $y^{\prime \prime}\left(\tau_{0}\right)>0$ from $\left(E_{y}\right)$, then $\tau_{0}$ is a point of local minimum. From $(2-16), \tau_{0}$ is unique, so that it is a minimum. Then $y>0, \lim _{\tau \rightarrow \infty} \zeta=\alpha, \lim _{\tau \rightarrow \ln R_{w}} Y / y=1$, and $\mathscr{T}_{[P]}$ stays in $2_{1}$ if $\alpha>0$, or goes from $2_{1}$ into $2_{3}$ if $\alpha<0$. The corresponding $w$ is of type (2).

For any point $P=(0, \xi), \xi>0$, the trajectory $\mathscr{T}_{[P]}$ goes through $P$ from $2_{1}$ into $2_{2}$, by Remark 2.1(i). Then $y$ has only one zero, and as above, it is decreasing on $\mathbb{R}$ and $\lim _{\tau \rightarrow \infty} y=-\infty$, and $\lim _{\tau \rightarrow \infty} \zeta=\alpha, \lim _{\tau \rightarrow \ln R_{w}} Y / y=1$. Thus $\mathscr{T}_{[P]}$ starts in $2_{1}$, then stays in $2_{2}$ if $\alpha<0$, and enters $2_{3}$ and stays in it if $\alpha>0$. The corresponding $w$ is of type (3).

It remains to prove the existence of a solution of type (1). If $\delta<N$, then $(0,0)$ is a saddle point. There exists a trajectory $\mathscr{T}_{1}$ converging to $(0,0)$ at $\infty$, with $y>0$, and $\lim _{\tau \rightarrow \infty} \zeta=\eta>0$, thus in $2_{1}$ near $\infty$, with $y^{\prime}<0$. As above, $y$ has no local maximum, it is increasing, so that $y>0$. If $\delta=N$, we consider the sets

$$
\begin{aligned}
& \mathscr{A}=\left\{P \in(0, \infty) \times \mathbb{R}: \mathscr{T}_{[P]} \cap \mathcal{M} \neq \varnothing\right\}, \\
& \mathscr{B}=\left\{P \in(0, \infty) \times \mathbb{R}: \mathscr{T}_{[P]} \cap\{(0, \xi): \xi>0\} \neq \varnothing\right\} .
\end{aligned}
$$


They are nonempty, and open, because the intersections are transverse. Since $\mathscr{T}_{r}$ is below $\mathcal{M}$, the sets $\mathscr{A}$ and $\mathscr{R}$ are contained in the domain $\mathscr{R}$ of $\mathscr{2}_{1} \cup \mathscr{2}_{2}$ above $\mathscr{T}_{r}$, and $\mathscr{A} \cup \mathscr{B} \neq \mathscr{R}$. As a result there exists at least a trajectory $\mathscr{T}_{1}$ above $\mathscr{T}_{r}$, which does not intersects $\mathcal{M}$ and the set $\{(0, \xi): \xi>0\}$. The corresponding $y$ is monotone. Suppose that $y$ is increasing, then $\lim _{\tau \rightarrow-\infty} y=0$; it is impossible since $\mathscr{T}_{1} \neq \mathscr{T}_{r}$. Then $y$ is decreasing, and $\lim _{\tau \rightarrow \infty} y=0$. In any case $w$ is of type (1), by Propositions 2.8 and 2.9. All the solutions are described, because any solution has at most one zero, and at most one extremum point. And $\mathscr{T}_{1}$ is unique when $\delta<N$.

\section{The case $\varepsilon=-1, \delta<\alpha$}

$$
N<\delta<\alpha
$$

Theorem 4.1. Assume $\varepsilon=-1$ and $N<\delta<\alpha$. Then all regular solutions of $\left(E_{w}\right)$ have constant sign and satisfy $S_{w}<\infty$. And $w \equiv \ell r^{-\delta}$ is a solution. There exist solutions satisfying any one of these characterizations:

(1) $w$ is positive, $\lim _{r \rightarrow 0} r^{\eta} w=c \neq 0$ if $N \geq 2$ (and $\lim _{r \rightarrow 0} w=a>0, \lim _{r \rightarrow 0} w^{\prime}$ $=b(a)<0$ if $N=1)$ and $\lim _{r \rightarrow \infty} r^{\delta} w=\ell ;$

(2) $w$ is positive, $\lim _{r \rightarrow 0} r^{\delta} w=\ell$ and $S_{w}<\infty$;

(3) $w$ has one zero, $\lim _{r \rightarrow 0} r^{\delta} w=\ell$ and $S_{w}<\infty$;

(4) $w$ is positive, $\lim _{r \rightarrow 0} r^{\alpha} w=L \neq 0$ and $\lim _{r \rightarrow \infty} r^{\delta} w=\ell$;

(5) $w$ is positive, $\lim _{r \rightarrow 0} r^{\alpha} w=L \neq 0$ and $S_{w}<\infty$;

(6) $w$ has one zero, $\lim _{r \rightarrow 0} r^{\alpha} w=L \neq 0$ and $S_{w}<\infty$;

(7) $w$ is positive, $\lim _{r \rightarrow 0} r^{\eta} w=c \neq 0$ if $N \geq 2$ (and $\lim _{r \rightarrow 0} w=a>0$ for any $a>0$ and $\lim _{r \rightarrow 0} w^{\prime}=b<0, b \neq b(a)$ if $\left.N=1\right)$, and $S_{w}<\infty$;

(8) $w$ has one zero and the same behavior;

(9) (only if $N=1$ ) $w$ is positive, $\lim _{r \rightarrow 0} w=a>0$, and $\lim _{r \rightarrow 0} w^{\prime}=b>0$, and $S_{w}<\infty$

Up to symmetry, all solutions are as above.

Proof. Here we still have three stationary points, $(0,0)$ is a source and $M_{\ell}$ a saddle point (see Figure 4). By Propositions 2.5 and 2.14, all regular solutions have constant sign and satisfy $S_{w}<\infty$. Also, $\mathscr{T}_{r}$ stays in $2_{4}$ by Remark 2.3 , and $\lim _{\tau \rightarrow \ln S_{w}} Y / y=-\infty$ by Proposition 2.15. Since $\alpha>0$, any solution $y$ has at most one zero, by Proposition 2.5, and $y$ is monotone near $\ln S_{w}$ (finite or not) and near $-\infty$, by Proposition 2.7. In the linearization near $M_{\ell}$ the eigenvectors $u_{1}=\left(v(\alpha), \lambda_{1}-\delta\right)$ and $u_{2}=\left(-v(\alpha), \delta-\lambda_{2}\right)$ form a positively oriented basis, where 

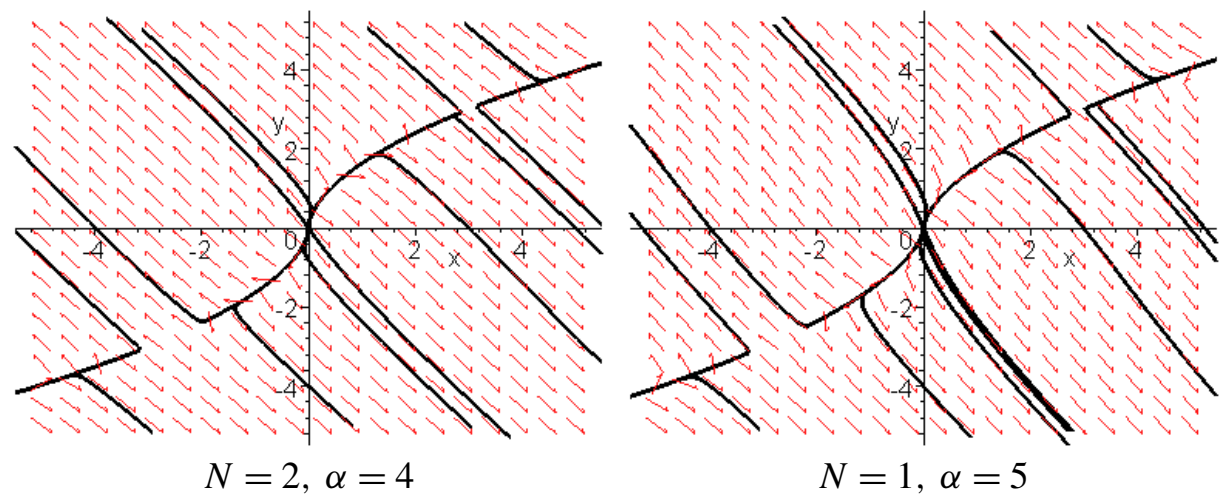

Figure 4. Theorem 4.1: $\varepsilon=-1, N<\delta=3<\alpha$.

now $v(\alpha)<0$ and $\lambda_{1}<\delta<\lambda_{2}$; thus $u_{1}$ points toward $2_{3}$ and $u_{2}$ points toward $2_{4}$. There exist four particular trajectories converging to $M_{\ell}$ near $\pm \infty$, namely:

- $\mathscr{T}_{1}$ converging to $M_{\ell}$ at $\infty$, with tangent vector $u_{1}$. Here $y$ is increasing near $\infty$, and as long as $y>0$; indeed, if there exists a minimal point $\tau,\left(E_{y}\right)$ shows that $y(\tau)>\ell$. And $\mathscr{T}_{1}$ stays in $\mathscr{2}_{1}$ on $\mathbb{R}$, by Remark 2.1(i) on page 211 . Therefore $\mathscr{T}_{1}$ converges to $(0,0)$ at $-\infty$, and $w$ is of type $(1)$, where $b(a)$ is a function of $a$, by the note on page 210 .

- $\mathscr{T}_{2}$ converging to $M_{\ell}$ at $-\infty$, with tangent vector $u_{2}$. Here again $y^{\prime}>0$ as long as $y>0$. Also $Y^{\prime}<0$ near $-\infty$, and $Y$ is decreasing as long as $Y>0$ : if there exists a minimal point of $Y$ in $2_{1},\left(E_{Y}\right)$ shows that $Y(\tau)>(\delta \ell)^{p-1}$. But $(y, Y)$ cannot stay in $2_{1}$, as this would imply $\lim _{\tau \rightarrow \infty} y=\infty$, which is impossible by Proposition 2.8. Thus $\mathscr{T}_{2}$ enters $\mathscr{2}_{4}$ at some point $\left(\xi_{2}, 0\right)$ with $\xi_{2}>0$ and stays in it since $y^{\prime}>0$. Thus $S_{w}<\infty$ and $\lim _{\tau \rightarrow \infty} Y / y=-1$, and $w$ is of type (2).

- $\mathscr{T}_{3}$ converging to $M_{\ell}$ at $-\infty$, with tangent vector $-u_{2}$. Here again $y^{\prime}<0$ as long as $y>0$. And $Y^{\prime}>0$ as long as $Y>0$; thus $Y^{\prime}>0$ on $\mathbb{R}$. Then again $(y, Y)$ cannot stay in $\mathscr{2}_{1}$, so $y$ has a unique zero, and $\mathscr{T}_{3}$ enters $\mathscr{2}_{2}$ at some point $\left(0, \xi_{3}\right)$ with $\xi_{3}>0$ and stays in it. Hence $S_{w}<\infty$ and $\lim _{\tau \rightarrow \infty} Y / y=-1$, and $w$ is of type (3).

- $\mathscr{T}_{4}$ converging to $M_{\ell}$ at $\infty$, with tangent vector $-u_{1}$. In the same way, $y$ is decreasing near $\infty$, and $y$ is everywhere decreasing: if there exists a maximal point $\tau$, then $y(\tau)<\ell$ by $\left(E_{y}\right)$. Then $Y$ stays positive, thus $\mathscr{T}_{4}$ stays in $\mathscr{2}_{1}$. By Proposition 2.8, $\lim _{\tau \rightarrow-\infty} y=\infty$ and $\lim _{\tau \rightarrow-\infty} \zeta=\alpha$, so $w$ is of type (4).

Next we describe all the other trajectories $\mathscr{T}_{[P]}$ with one point $P$ in the domain $\mathscr{R}$ above $\mathscr{T}_{r} \cup\left(-\mathscr{T}_{r}\right)$. 
If $P=(\varphi, 0)$ with $\varphi>\xi_{2}$, then $\mathscr{T}_{[P]}$ stays in $2_{4}$ after $P$, because it cannot meet $\mathscr{T}_{2}$; before $P$ it stays in $\mathscr{2}_{1}$, by Remark 2.1(i). Thus again $S_{w}=\infty$, and $\lim _{\tau \rightarrow-\infty} \zeta=\alpha>0$, and $y$ has a unique minimal point, and $w$ is of type (5). For any $P$ is in the domain delimited by $\mathscr{T}_{2}, \mathscr{T}_{4}$, the trajectory $\mathscr{T}_{[P]}$ is of the same type.

If $P=(0, \xi)$ with $\xi>\xi_{3}$, then $\mathscr{T}_{[P]}$ stays in $\mathscr{2}_{2}$ after $P$, in $2_{1}$ before $P$, since it cannot meet $\mathscr{T}_{2}, \mathscr{T}_{4}$. Then $\lim _{\tau \rightarrow-\infty} \zeta=\alpha>0$, and $S_{w}=\infty$, and $w$ is of type (6). If $P$ is in the domain delimited by $\mathscr{T}_{3}, \mathscr{T}_{4}$, then $\mathscr{T}_{[P]}$ is of the same type.

If $P=(\varphi, 0)$ with $\varphi \in\left(0, \xi_{2}\right)$, then $\mathscr{T}_{[P]}$ stays in $\mathscr{2}_{4}$ after $P$, in $\mathscr{2}_{1}$ before $P$; it cannot meet $\mathscr{T}_{r}$, thus $S_{w}<\infty$; and $\mathscr{T}_{[P]}$ converges to $(0,0)$ in $2_{1}$ at $-\infty$; thus $w$ is of type (7), by Theorem 2.2. If $P$ is in the domain delimited by $\mathscr{T}_{1}, \mathscr{T}_{2}, \mathscr{T}_{r}$, then $\mathscr{T}_{[P]}$ is of the same type.

If $P=(0, \xi)$ for some $\xi \in\left(0, \xi_{3}\right)$, then $\mathscr{T}_{[P]}$ stays in $\mathscr{2}_{2}$ after $P$, in $\mathscr{Q}_{1}$ before $P$; and $\mathscr{T}$ cannot meet $-\mathscr{T}_{r}$, so that $S_{w}<\infty$. Then $\mathscr{T}_{[P]}$ converges to $(0,0)$ in $\mathscr{2}_{1}$ at $-\infty$, and $w$ is of type (8).

If $P$ lies in the domain delimited by $\mathscr{T}_{1}, \mathscr{T}_{3}$ and $-\mathscr{T}_{r}$, either $y$ has one zero, and $\mathscr{T}_{[P]}$ is of the same type; or $y<0$ on $\mathbb{R}$, and $y^{\prime}=\delta y-Y^{1 /(p-1)}<0$. Hence $S_{w}<\infty$ and $\mathscr{T}_{[P]}$ converges to $(0,0)$ in $\mathscr{2}_{2}$ at $-\infty$. It implies $N=1$ (see Figure 4 , right), and $-w$ is of type (9), by Propositions 2.8 and 2.9; and such a solution does exist, by Theorem 2.2. Up to symmetry, all the solutions have been obtained. Here again, up to a scaling, the solutions $w$ of types (1)-(4) are unique.

\section{$\delta \leq \min (\alpha, N)($ apart from $\alpha=\delta=N)$}

Theorem 4.2. Suppose $\varepsilon=-1$ and $\delta \leq \min (\alpha, N)$ (apart from $\alpha=\delta=N)$. Then all regular solutions of $\left(E_{w}\right)$ have constant sign and a reduced domain $\left(S_{w}<\infty\right)$. There exist solutions satisfying any one of these characterizations:

(1) $w$ is positive, $\lim _{r \rightarrow 0} r^{\alpha} w=L \neq 0$ and $\lim _{r \rightarrow \infty} r^{\eta} w=c \neq 0$ if $\delta<N$, or (2-40) holds if $\delta=N<\alpha$;

(2) $w$ is positive, $\lim _{r \rightarrow 0} r^{\alpha} w=L \neq 0$ if $\delta<\alpha$, or (2-39) holds if $\alpha=\delta<N$, and $S_{w}<\infty$

(3) w has one zero and the same behavior.

Up to symmetry, all solutions are as above.

Proof. Here $(0,0)$ is the only one stationary point, and $N \geq 2$ (Figure 5). By Propositions 2.5 and 2.14, all regular solutions have constant sign, and $S_{w}<$ $\infty$. Moreover $w^{\prime}>0$ near 0 , by Theorem 2.2; and $w$ can only have minimal points, by Remark 2.3, so $w^{\prime}>0$ on $\left(0, S_{w}\right)$. In other words, $\mathscr{T}_{r}$ stays in $2_{4}$, and $\lim _{\tau \rightarrow \ln S_{w}} Y / y=-1$. By Propositions 2.5 and 2.7, any solution $y$ has at most one zero and is monotone at the extremities. By Proposition 2.8, apart from $\mathscr{T}_{r}$, any 

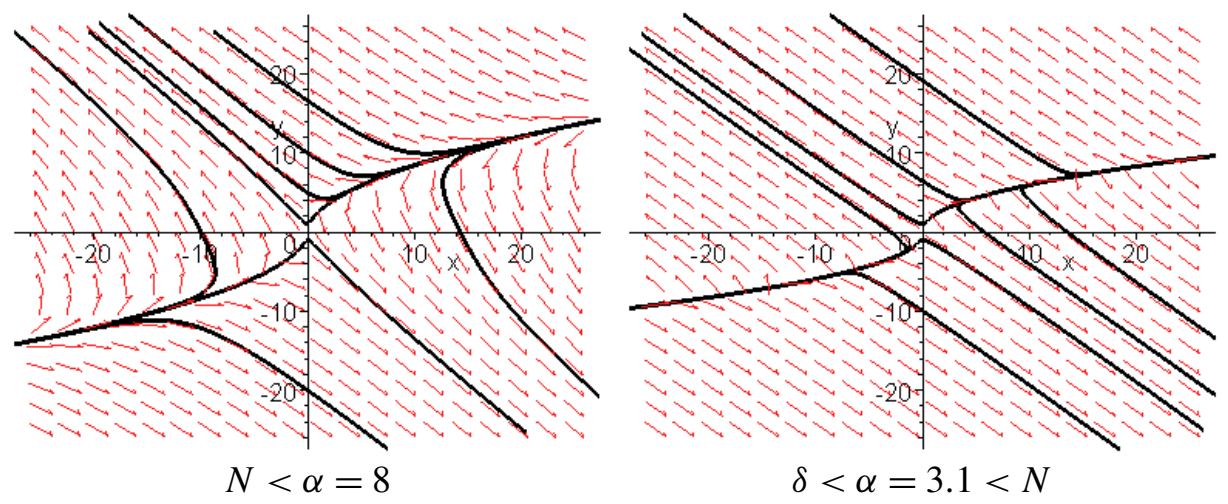

Figure 5. Theorem 4.2: $\varepsilon=-1, \delta=3<N=4$.

trajectory $\mathscr{T}$ satisfies $\lim _{\tau \rightarrow-\infty}|y|=\infty$, and so $\lim _{\tau \rightarrow-\infty} \zeta=\alpha>0$; hence $\mathscr{T}$ starts from $2_{1}$ or $2_{3}$ at $-\infty$.

For any $P=(\varphi, 0)$ with $\varphi>0$, the trajectory $\mathscr{T}_{[P]}$ goes from $2_{1}$ into $\mathscr{2}_{4}$ at $P$, by Remark 2.1(i) on page 211; it stays in $2_{4}$ after $P$, since it cannot meet $\mathscr{T}_{r}$; and it stays in $\mathscr{2}_{1}$ before $P$ : it cannot start from $2_{3}$, because it does not meet $-\mathscr{T}_{r}$. Thus $y$ remains positive and $w$ is of type (2).

For any $P=(0, \xi)$ with $\xi>0, \mathscr{T}_{[P]}$ goes from $\mathscr{2}_{1}$ into $\mathscr{2}_{2}$ by the same remark; thus $\mathscr{T}_{[P]}$ stays in $\mathscr{2}_{2}$ after $P$, since it cannot meet $-\mathscr{T}_{r}$, and in $\mathscr{2}_{1}$ before $P$, and $w$ is of type (3).

It remains to prove the existence of solutions of type (1). If $\delta<N$, the origin is a saddle point, so there exists a trajectory $\mathscr{T}_{1}$ converging to $(0,0)$ at $\infty$; and $\lim _{\tau \rightarrow \infty} \zeta=\eta>0$, by Proposition 2.8. Thus $\mathscr{T}_{1}$ lies in $\mathscr{2}_{1}$ for large $\tau$, and stays there, because $2_{1}$ is backward invariant. The conclusion follows. If $\delta=N$, we consider the sets

$$
\begin{aligned}
& \mathscr{A}=\left\{P \in \mathscr{Q}_{1}: \mathscr{T}_{[P]} \cap\{(\varphi, 0): \varphi>0\} \neq \varnothing\right\}, \\
& \mathscr{B}=\left\{P \in \mathscr{Q}_{1}: \mathscr{T}_{[P]} \cap\{(0, \xi): \xi>0\} \neq \varnothing\right\} .
\end{aligned}
$$

They are nonempty and open, since the vector field is transverse at $(\varphi, 0)$ and $(0, \xi)$; thus $\mathscr{A} \cup \mathscr{B} \neq \mathscr{2}_{1}$. Hence there exists a trajectory $\mathscr{T}_{1}$ staying in $\mathscr{2}_{1}$; therefore $S_{w}=\infty$ and $\mathscr{T}_{1}$ converges to $(0,0)$ at $\infty$, and $w$ is of type (1), by Proposition 2.9. All solutions have been described, up to symmetry.

\section{The case $\varepsilon=1, \delta \leq \alpha$$$
N \leq \delta \leq \alpha
$$

Theorem 5.1. Assume $\varepsilon=1, N \leq \delta \leq \alpha$ and $\alpha \neq N$. 

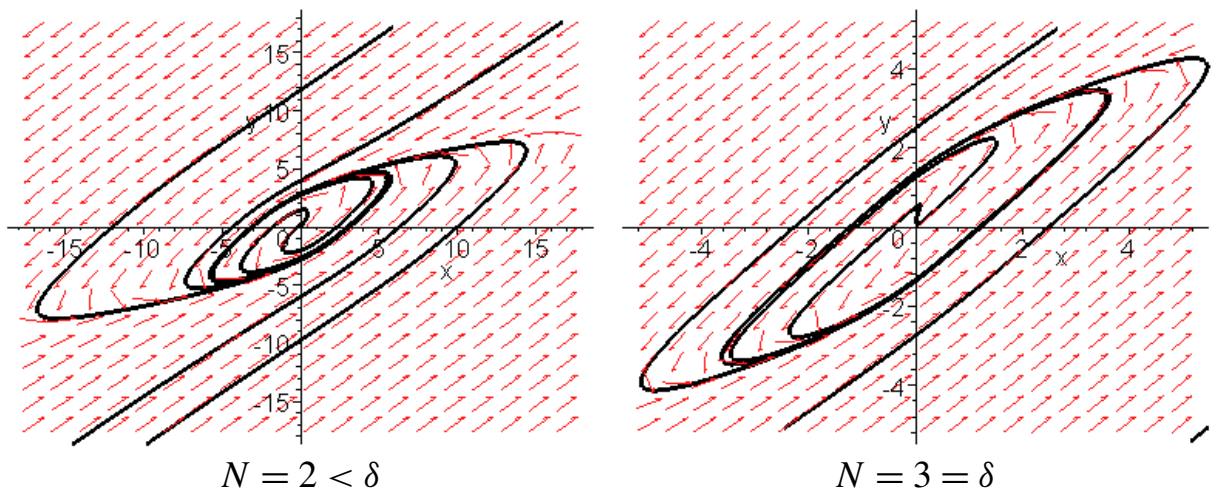

Figure 6. Theorem 5.1: $\varepsilon=1,<\delta=3<\alpha=3.5$.

(i) There exists a cycle surrounding $(0,0)$, and thus also solutions $w$ of $\left(E_{w}\right)$ with changing sign and such that $r^{\delta} w$ is periodic in $\ln r$. All other solutions $w$, in particular the regular ones, are oscillating near $\infty$, and $r^{\delta} w$ is asymptotically periodic in $\ln r$. There exist solutions $w$ such that $\lim _{r \rightarrow 0} r^{\eta} w=c \neq 0$ if $2 \leq N<\delta$ and (2-40) holds if $N=\delta$, or (2-41) holds if $N=1$.

(ii) There exist solutions such that $R_{w}>0$, or $\lim _{r \rightarrow 0} r^{\alpha} w=L \neq 0$ if $\alpha \neq \delta$, or (2-39) holds if $\alpha=\delta$.

Proof. (i) Here $(0,0)$ is the only stationary point. From Proposition 2.8, any trajectory is bounded and $y$ is oscillating around 0 near $\infty$.

First assume $N<\delta<\alpha$ (Figure 6, left). Then $(0,0)$ is a source and all trajectories have a limit cycle at $\infty$ or are periodic. In particular there exists at least one cycle, with orbit $\mathcal{O}_{p}$. The trajectory $\mathscr{T}_{r}$ has a limit cycle $\mathcal{O} \subseteq O_{p}$. There exist also trajectories $\mathscr{T}_{s}$ starting from $(0,0)$ with an infinite slope, such that $\lim _{r \rightarrow 0} r^{\eta} w=$ $c \neq 0$ if $N \geq 2$ or (2-41) if $N=1$, and all the $\mathscr{T}_{s}$ have the same limit cycle $\mathbb{O}$.

Next assume $N=\delta<\alpha$ (Figure 6 , right). Then $\mathscr{T}_{r}$ cannot converge to $(0,0)$, since it would intersect itself. Thus again the limit set at $\infty$ is a closed orbit 0 . No trajectory can converge to $(0,0)$ at $\infty$, as it would spiral around this point and hence intersect $\mathscr{T}_{r}$. Consider any trajectory $\mathscr{T} \neq \mathscr{T}_{r}$ in the connected component of 0 containing $(0,0) . \mathcal{T}$ is bounded, so its limit set at $-\infty$ is $(0,0)$ or a closed orbit. The second case is impossible, since $\mathscr{T}$ does not meet $\mathscr{T}_{r}$. Thus $\mathscr{T}$ is of the form $\mathscr{T}_{s}$, and the corresponding $w$ satisfies $(2-40)$.

(ii) By Theorem 2.21, all cycles are contained in a ball $B$ of $\mathbb{R}^{2}$. Take any point $P_{0}$ exterior to $B$. Then $\mathscr{T}_{\left[P_{0}\right]}$ has a limit cycle at $\infty$ contained in $B$. If it has a limit cycle at $-\infty$, it is contained in $B$, so $\mathscr{T}_{\left[P_{0}\right]}$ is contained in $B$, which is impossible. Thus $y$ has constant sign near $\ln R_{w}$. By Proposition 2.8, either $R_{w}>0$ or $y$ is defined near $-\infty$. 


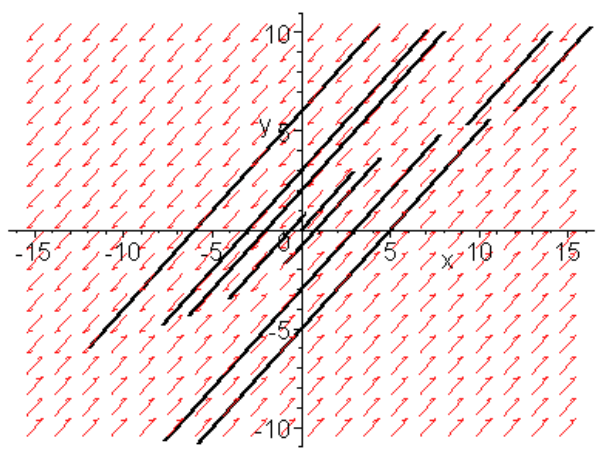

Figure 7. Theorem 5.2: $\varepsilon=1, \alpha=\delta=N=3$.

Theorem 5.2. Assume $\varepsilon=1$ and $\alpha=\delta=N$. All regular solutions of $\left(E_{w}\right)$ have constant sign, and are given by (1-6). For any $k \in \mathbb{R}, w(r)=k r^{-N}$ is a solution. There exist solutions satisfying any one of these characterizations:

(1) $w$ is positive, $\lim _{r \rightarrow 0} r^{N} w=c_{1}>0, \lim _{r \rightarrow 0} r^{N} w=c_{2}>0\left(c_{2} \neq c_{1}\right)$;

(2) $w$ has one zero, $\lim _{r \rightarrow 0} r^{N} w=c_{1}>0$ and $\lim _{r \rightarrow \infty} r^{N} w=c_{2}<0$;

(3) $w$ is positive, $R_{w}>0$, and $\lim _{r \rightarrow 0} r^{N} w=c \neq 0$;

(4) $w$ has one zero and the same behavior.

Up to symmetry, all solutions are as above.

Proof. Since $\alpha=N$, equation ( $\left.E_{w}\right)$ admits the first integral (1-5), so $J_{N} \equiv C$ for $C \in \mathbb{R}$. We gave in (1-6) the regular (Barenblatt) solutions for the case $C=0$. Since $\delta=N$, (1-5) is equivalent to the equation $Y \equiv y-C$, by (2-12) (refer to Figure $7)$. For any $k \in \mathbb{R},(y, Y) \equiv\left(k,|N k|^{p-2} N k\right)$ is a solution of the system $(S)$ located on the curve $M$, so that $w(r)=k r^{-N}$ is a solution. Any solution has at most one zero, by Proposition 2.5. By Propositions 2.8 and 2.10, any trajectory converges to a point $\left(k,|N k|^{p-2} N k\right)$ of $M$ at $\infty$. Let $\bar{C}<0$ be such that the line $Y=y-\bar{C}$ is tangent to $M$. For any $C \in(\bar{C}, 0)$, the line $Y=y-C$ cuts $M$ at three points $k_{1}<0<k_{2}<k_{3}$. And $y^{\prime}>0$ if the trajectory is below $M$ and $y^{\prime}<0$ if it is above $\mu$. We find two solutions $y$ defined on $\mathbb{R}$ : one is positive and $\lim _{\tau \rightarrow-\infty} y=k_{2}$, $\lim _{\tau \rightarrow-\infty} y=k_{3}$, and the other has one zero. All other solutions satisfy $R_{w}>0$, $\lim _{\tau \rightarrow \ln R_{w}} Y / y=1$; some of them are positive, the others have one zero.

\section{$\delta<\min (\alpha, N)$}

Here the system has three stationary points: $(0,0)$ is a saddle point, while $M_{\ell}$, $M_{\ell}^{\prime}$ are sinks if $\delta \leq N / 2$, or $N / 2<\delta$ and $\alpha<\alpha^{*}$, and sources when $N / 2<$ $\delta$ and $\alpha>\alpha^{*}$, and node points whenever $\alpha \leq \alpha_{1}$ or $\alpha_{2} \leq \alpha$, where $\alpha_{1}, \alpha_{2}$ are defined in (2-48) (recall that $\alpha_{1}$ can be greater or less than $\eta$ ). This case is one 
of the most delicate, since two types of periodic trajectories can appear, either surrounding $(0,0)$, corresponding to changing sign solutions, or located in $2_{1}$ or $2_{3}$, corresponding to solutions of constant sign. Notice that $\delta<N$ implies $\delta<$ $N<\eta$ by (1-2), and $N / 2<\delta$ implies $\eta<\alpha^{*}$ by $(2-32)$.

Remark 5.3. (i) $\mathscr{T}_{r}$ starts in $\mathscr{2}_{1}$. Since $(0,0)$ is a saddle point, Propositions 2.8 and 2.9 show there is a unique trajectory $\mathscr{T}_{s}$ converging to $(0,0)$, residing in $\mathscr{2}_{1}$ for large $\tau$, having an infinite slope at $(0,0)$, and satisfying $\lim _{r \rightarrow 0} r^{\eta} w=c>0$. Moreover if $\mathscr{T}_{r}$ does not stay in $\mathscr{2}_{1}$, then $\mathscr{T}_{s}$ stays in it, and is bounded and contained in the domain delimited by $\mathscr{2}_{1} \cap \mathscr{T}_{r}$, by Remark 2.1(i). Thus if $\mathscr{T}_{r}$ is homoclinic, it stays in $2_{1}$.

(ii) Any trajectory $\mathcal{T}$ is bounded near $\infty$, by Propositions 2.8 and 2.12. From the strong form of the Poincaré-Bendixson theorem [Hubbard and West 1995, p. 239], any trajectory $\mathcal{T}$ bounded at $\pm \infty$ either converges to $(0,0)$ or $\pm M_{\ell}$, or its limit set $\Gamma_{ \pm}$at $\pm \infty$ is a cycle, or it is homoclinic hence $\mathscr{T}=\mathscr{T}_{r}$ and $\Gamma_{ \pm}=\overline{\mathscr{T}}_{r}$ (indeed, for any $P \in \Gamma_{ \pm}, \mathscr{T}_{[P]}$ converges at $\infty$ and $-\infty$ to $(0,0)$ or $\pm M_{\ell}$; if one of them is $\pm M_{\ell}$, then $\pm M_{\ell} \in \overline{\mathscr{T}}_{[P]} \subset \Gamma_{ \pm}$, and $M_{\ell}$ is a source or a sink, so $\mathcal{T}$ converges to $\pm M_{\ell}$; otherwise $\mathscr{T}$ is homoclinic and $\mathscr{T}_{[P]}=\mathscr{T}_{r}$ ).

(iii) If there exists a limit cycle around $(0,0)$, then by (2-42) this cycle also surrounds the points $\pm M_{\ell}$.

We begin with the case $\alpha \leq \eta$, where there exists no cycle in $2_{1}$ and no homoclinic orbit, by Theorem 2.20.

Theorem 5.4. Assume that $\varepsilon=1$ and $\delta<\min (\alpha, N)$, and $\alpha \leq \eta$. Then all regular solutions of $\left(E_{w}\right)$ have constant sign, and $\lim _{r \rightarrow \infty} r^{\delta}|w(r)|=\ell$. And $w(r)=\ell r^{-\delta}$ is a solution.

If $\alpha<\eta$, there exist solutions satisfying any one of these characterizations:

(1) $w$ is positive, $\lim _{r \rightarrow 0} r^{\alpha} w=L$ and $\lim _{r \rightarrow \infty} r^{\delta} w=\ell$;

(2) $w$ is positive, $R_{w}>0$ and $\lim _{r \rightarrow \infty} r^{\eta} w=c>0$;

(3) $w$ is positive, $R_{w}>0$ and $\lim _{r \rightarrow \infty} r^{\delta} w=\ell$;

(4) $w$ has one zero, $R_{w}>0$ and $\lim _{r \rightarrow \infty} r^{\delta} w=\ell$;

If $\alpha=\eta$, then $w=C r^{-\eta}$ is a solution and there exist solutions of type (4), but not of type (2) or (3).

Proof. By Proposition 2.5 and Remark 2.3, $\mathscr{T}_{r}$ stays in $\mathscr{2}_{1}$ and converges to $M_{\ell}$ at $\infty$; indeed there is no cycle in $2_{1}$, by Propositions $2.8,2.12$ and 2.20.

(i) Assume $\alpha<\eta$ (Figure 8, left). Consider any trajectory in $2_{1}$. Then $Y_{\alpha}>0$. If there exists $\tau$ such that $Y_{\alpha}^{\prime}(\tau)=0$, at this point $Y_{\alpha}^{\prime \prime}(\tau) \geq 0$ by (2-36), and $\tau$ 

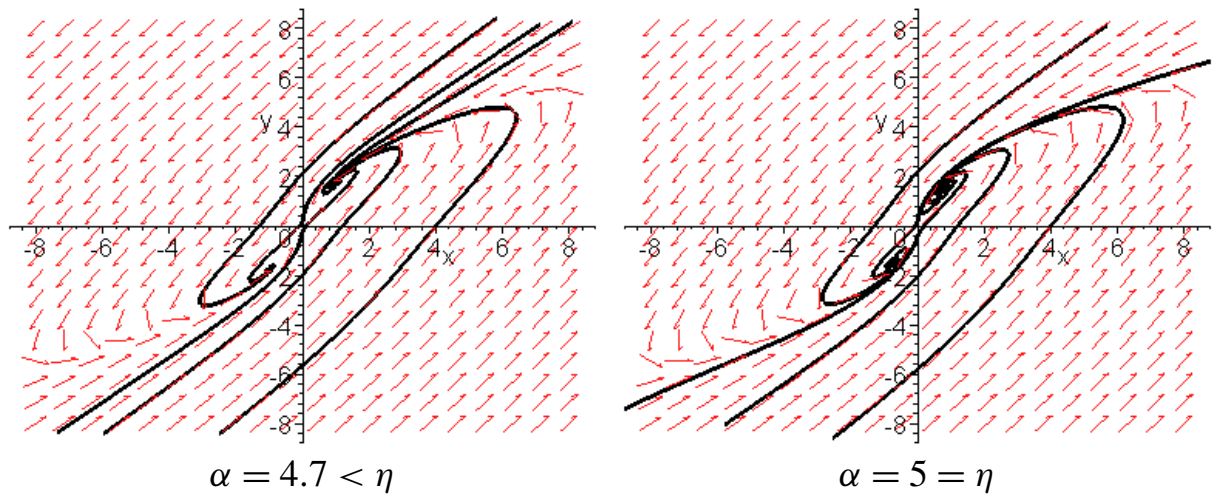

Figure 8. Theorem 5.4: $\varepsilon=1, \delta=3<N=4<\eta=5$.

is a local minimum. $\mathscr{T}_{r}$ satisfies $\lim _{\tau \rightarrow-\infty} Y_{\alpha}=0$, and so $Y_{\alpha}^{\prime}>0$ on $\mathbb{R}$. This is equivalent to $\alpha y>Y^{1 /(p-1)}+(p-1)(\eta-\alpha) Y$. Therefore $\mathscr{T}_{r}$ stays strictly below the curve

$$
\mu_{\alpha}=\left\{(y, Y) \in 2_{1}: \alpha y=Y^{1 /(p-1)}+(p-1)(\eta-\alpha) Y\right\} .
$$

First consider $\mathscr{T}_{s}$. Since $\alpha<\eta$, this trajectory satisfies $\lim _{\tau \rightarrow \infty} Y_{\alpha}=0$. Then $Y_{\alpha}^{\prime}<0$ on $\left(\ln R_{w}, \infty\right)$, so $\mathscr{T}_{s}$ stays strictly above $\mu_{\alpha}$. Hence it stays above $\mathcal{M}$ : indeed, if it meets $M$ at a first point $\left(y_{1},\left(\delta y_{1}\right)^{p-1}\right)$, the function $y$ has a maximum at this point. Thus by $(2-16)$, we have $\ell<y_{1}$ and

$$
(\alpha-\delta) y_{1}^{2-p}=\delta^{p-1}(p-1)(\eta-\alpha)<\delta^{p-1}(p-1)(\eta-\delta),
$$

contradicting (1-2) and (1-4). This shows that $y^{\prime}<0$. Suppose that $y$ is defined on $\mathbb{R}$; then $\lim _{\tau \rightarrow-\infty} y=\infty$ and $\lim _{\tau \rightarrow-\infty} \zeta=\alpha$. If $\zeta^{\prime}>0$ on $\mathbb{R}$, then $\zeta(\mathbb{R})=(\alpha, \eta)$, which contradicts (2-9). Then $\zeta$ has at least one extremal point $\tau$, and $\zeta(\tau)$ is exterior to $(\alpha, \eta)$, by (2-9); if it is a minimum, $\zeta(\tau)>\alpha$ by (2-18), since $y^{\prime}<0$, and if it is a maximum, $\zeta(\tau)<\alpha$. Thus we reach again a contradiction. Therefore $R_{w}>0$ and $\lim _{\tau \rightarrow \ln R_{w}} Y / y=1$, and the corresponding $w$ is of type (2).

For any $P=(\varphi, 0), \varphi>0$, the trajectory $\mathscr{T}_{[P]}$ stays in $\mathscr{2}_{1}$ after $P$. The solution $(y, Y)$ originating at $P$ at time 0 satisfies $Y_{\alpha}(0)=0$; hence $Y_{\alpha}^{\prime}(\tau)>0$ for any $\tau \geq 0$. Thus $\mathscr{T}_{[P]}$ stays below $\mathcal{M}_{\alpha}$. Moreover it enters $2_{4}$ as $\tau$ decreases. But $y^{\prime}>0$ in $2_{4}$, by $(S)$; thus $\mathscr{T}_{[P]}$ does not stay in $2_{4}$, by Proposition 2.8; it goes into $2_{3}$ and must stay there because it cannot meet $-\mathscr{T}_{s}$. This shows that $R_{w}>0$ and $y$ has precisely one zero, and $w$ is of type (4).

Next consider any trajectory $\mathscr{T}_{\left[P_{1}\right]}$ going through some point $P_{1}=\left(y_{1}, Y_{1}\right)$ in $2_{1}$, lying below $\mathscr{T}_{s}$ and such that $\alpha y_{1}<Y_{1}^{1 /(p-1)}$. Such a trajectory exists because $y=Y$ is an asymptotic direction of $\mathscr{T}_{s}$. Let $(y, Y)$ be the solution issuing from $P_{1}$ at time 0 . Suppose $y$ is defined on $\mathbb{R}$; then $\lim _{\tau \rightarrow-\infty} y=\infty$ and $\lim _{\tau \rightarrow-\infty} \zeta=\alpha$. 
Also, $\zeta(0)>\alpha$. Then $\zeta>\delta$ on $(-\infty, 0)$ : otherwise there would exist $\tau<0$ such that $\zeta(\tau)=\alpha$ and $\zeta^{\prime}(\tau) \geq 0$, contradicting (2-9). Thus $y^{\prime}<0$ on $\left(-\infty, \tau_{1}\right)$. Either $\zeta^{\prime}>0$ on $(-\infty, 0)$, in which case $\zeta>\eta>0$ by (2-9), which is impossible; or $\zeta$ has at least an extremal point $\tau$. If it is a minimum, then $\zeta(\tau)>\alpha$ from $(2-18)$; if it is a maximum, then $\zeta(\tau)<\alpha$; and again we reach a contradiction. Therefore $R_{w}>0$, and the trajectory stays in $2_{1}$ and converges to $M_{\ell}$, because there is no cycle in $2_{1}$, by Theorem 2.20. Hence $w$ is of type (3).

Let $O$ be the domain of $2_{1}$ bounded above by $\mathscr{T}_{s}$. It is forward invariant. Any trajectory going through any point of $\mathcal{O}$ converges to $M_{\ell}$ at $\infty$. Either it meets the axis $Y=0$ at some point $(\xi, 0)$ with $\xi>0$, or it stays in $\mathcal{O}$, satisfies $R_{w}>0$ and $\lim _{\tau \rightarrow \ln R_{w}} T / y=1$, and meets $M_{\alpha}$, since $M_{\ell}$ lies strictly below $M_{\alpha}$. Let

$$
\begin{aligned}
& \mathscr{A}=\left\{P \in \mathbb{O}: \mathscr{T}_{[P]} \cap\{(\varphi, 0): \varphi>0\} \neq \varnothing\right\}, \\
& \mathscr{B}=\left\{P \in \mathcal{O}: \mathscr{T}_{[P]} \cap \mathcal{M}_{\alpha} \neq \varnothing\right\} .
\end{aligned}
$$

These sets are nonempty and open: indeed, one can check that the intersection with $\mathcal{M}_{\alpha}$ is transverse, because $\alpha \neq \eta$. Thus $\mathscr{A} \cup \mathscr{B} \neq 0$, so there exists a trajectory $\mathscr{T}_{1}$ with $w$ of type (1).

(ii) Assume $\alpha=\eta$ (Figure 8, right). There is no positive solution with $R_{w}>0$, thus no solution of type (2) or (3). Indeed all the trajectories stay below $\mathscr{T}_{s}$, and $\mathscr{T}_{s}$ is defined by the equation $\zeta \equiv \eta$, meaning that $w \equiv C r^{-\eta}$, or equivalently $Y_{\eta} \equiv C$; thus $Y_{\eta}^{\prime} \equiv 0$ and $\mathscr{T}_{s}=\mathcal{M}_{\eta}$. Consider any trajectory $\mathscr{T}_{[P]}$ going through some point $P=(\varphi, 0)$ with $\varphi>0$, and the solution $(y, Y)$ issuing from $P$ at time 0 . Then $Y_{\eta}(0)=0$ and $Y_{\eta}<0$, so $Y_{\eta}^{\prime}=\eta y-|Y|^{(2-p) /(p-1)} Y>0$ on $(-\infty, 0)$, seeing that $\mathscr{T}_{[P]}$ does not meet $-\mathscr{T}_{s}$. Suppose $R_{w}=0$. Then $\mathscr{T}_{[P]}$ starts from 23, with $\lim _{\tau \rightarrow-\infty} \zeta=\alpha=\eta$. Then $\lim _{\tau \rightarrow-\infty} y_{\eta}=L<0$; thus $\lim _{\tau \rightarrow-\infty} Y_{\eta}=$ $-(\alpha|L|)^{(2-p) /(p-1)}$. A straightforward computation gives

$$
Y_{\eta}^{\prime \prime}=Y_{\eta}^{\prime}\left(N-\frac{1}{p-1}|Y|^{(2-p) /(p-1)}\right) .
$$

This leads to $Y_{\eta}^{\prime \prime}<0$ near $-\infty$, which is impossible; thus $R_{w}<\infty$ and $w$ is of type (4).

Remarks. (i) For $\alpha \leq \eta$, both trajectories $\mathscr{T}_{r}$ and $\mathscr{T}_{s}$ stay in $\mathscr{2}_{1}$.

(ii) When $\alpha \leq N$, one can verify that the regular positive solution $y$ is increasing and $y \leq \ell$ on $\mathbb{R}$, so $r^{\delta} w(r) \leq \ell$ for any $r \geq 0$.

(iii) When $\alpha=N$, we have $\mathscr{T}_{r}=\{(\xi, \xi): \xi \in[0, \ell)\}$, and the corresponding solutions $w$ are given by (1-6) with $K>0$. And $\left.\mathscr{T}_{3}=\{(\xi, \xi): \xi>\ell)\right\}$ is a trajectory corresponding to particular solutions $w$ of type (3), given by (1-6) with $K<0$. 
Next we come to the most interesting case, where $\eta<\alpha$.

Lemma 5.5. Assume $\varepsilon=1, \delta<\min (\alpha, N)$ and $\eta<\alpha$. If $N / 2<\delta$ and $\alpha<\alpha^{*}$ and $\mathscr{T}_{s}$ stays in $2_{1}, \mathscr{T}_{s}$ has a limit cycle at $-\infty$ in $2_{1}$ or is homoclinic. If $\delta \leq N / 2$, then $\mathscr{T}_{s}$ does not stay in $2_{1}$.

Proof. In any case $M_{\ell}$ is a sink, so $\mathscr{T}_{s}$ cannot converge to $M_{\ell}$ at $-\infty$. Suppose $\mathscr{T}_{s}$ has no limit cycle in $2_{1}$, and is not homoclinic and stays in $2_{1}$. (This happens when $\delta \leq N / 2$, by Proposition 2.11.) Then either $\lim _{\tau \rightarrow-\infty} y=\infty$ and $\lim _{r \rightarrow 0} r^{\alpha} w=$ $\Lambda \neq 0$, or $R_{w}>0$. In either case, for any $d \in(\eta, \alpha)$, the function $y_{d}(\tau)=r^{d} w=$ $r^{d-\delta} y$ satisfies $\lim _{\tau \rightarrow \ln R_{w}} y_{d}=\infty=\lim _{\tau \rightarrow \infty} y_{d}$. Then it has a minimum point, contradicting (2-5).

Theorem 5.6. Assume $\varepsilon=1$ and $N / 2<\delta<\min (\alpha, N)$. Then $w(r)=\ell r^{-\delta}$ is still a solution.

(i) There exists a (maximal) critical value $\alpha_{\text {crit }}$ of $\alpha$, such that

$$
\max \left(\eta, \alpha_{1}\right)<\alpha_{\text {crit }}<\alpha^{*}
$$

and the regular trajectory is homoclinic: all regular solutions of $\left(E_{w}\right)$ have constant sign and satisfy $\lim _{r \rightarrow \infty} r^{\eta} w=c \neq 0$.

(ii) For any $\alpha \in\left(\alpha_{\text {crit }}, \alpha^{*}\right)$, there does exist a cycle in $\mathscr{2}_{1}$, in other words there exist positive solutions $w$ such that $r^{\delta} w$ is periodic in $\ln r$. There exist positive solutions such that $r^{\delta} w$ is asymptotically periodic in $\ln r$ near 0 and $\lim _{r \rightarrow \infty} r^{\delta} w=\delta$. There exist positive solutions such that $r^{\delta} w$ is asymptotically periodic in $\ln r$ near 0 and $\lim _{r \rightarrow \infty} r^{\eta} w=c \neq 0$.

(iii) For any $\alpha \geq \alpha^{*}$ there does not exist such a cycle, but there exist positive solutions such that $\lim _{r \rightarrow 0} r^{\delta} w=\ell$ and $\lim _{r \rightarrow \infty} r^{\eta} w=c>0$.

(iv) For any $\alpha>\alpha_{\text {crit, }}$, there exists also a cycle, surrounding $(0,0)$ and $\pm M_{\ell}$, thus $r^{\delta} w$ is changing sign and periodic in $\ln r$. All regular solutions change signs and are oscillating at $\infty$, and $r^{\delta} w$ is asymptotically periodic in $\ln r$. There exist solutions such that $R_{w}>0$, or $\lim _{r \rightarrow 0} r^{\alpha} w=L \neq 0$, and oscillating at $\infty$, and $r^{\delta} w$ is asymptotically periodic in $\ln r$.

Proof. (i) For any $\alpha \in\left(\alpha_{1}, \alpha_{2}\right)$ such that $\eta \leq \alpha$, we have by Remark 5.3 three possibilities for the regular trajectory $\mathscr{T}_{r}$ :

- $\mathscr{T}_{r}$ converges to $M_{\ell}$ and spirals around it, or else it has a limit cycle in $\mathscr{2}_{1}$ around $M_{\ell}$. Then $\mathscr{T}_{r}$ meets the set $\mathscr{E}=\left\{(\ell, Y): Y>(\delta \ell)^{p-1}\right\}$ at a first point $\left(\ell, Y_{r}(\alpha)\right)$. Note that $\ell$ and $\mathscr{E}$ depend continuously on $\alpha$. Then $\mathscr{T}_{s}$ meets $\mathscr{E}$ at some last point $\left(\ell, Y_{S}(\alpha)\right)$ such that $Y_{S}(\alpha)-Y_{r}(\alpha)>0$. See Figure 9, top left. 


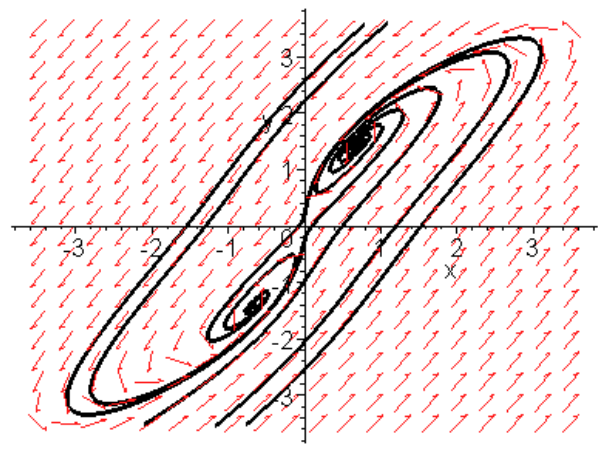

$N<\eta=5<\alpha=5.1$

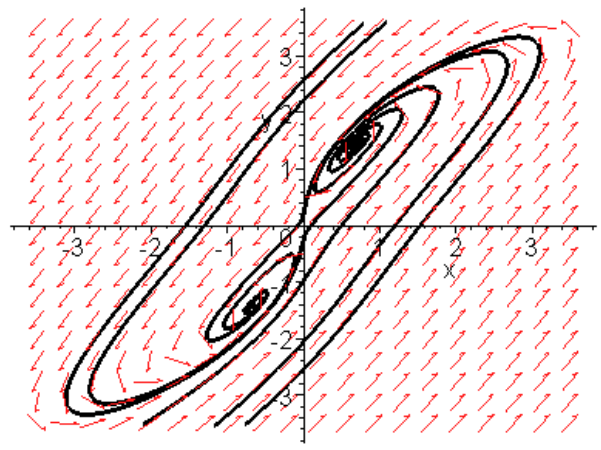

$N<\alpha=5.9<\alpha^{*}=6$

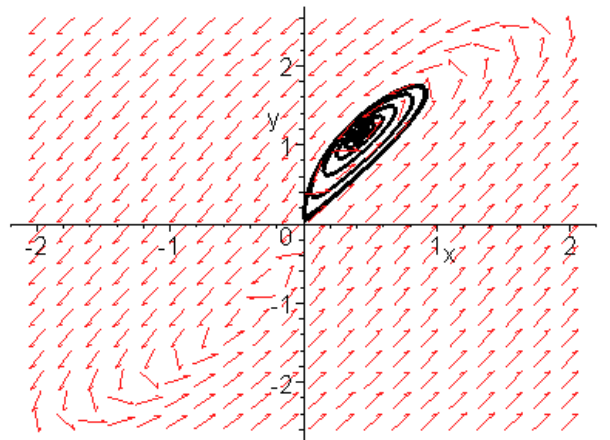

$N<\eta=5<\alpha=5.62$

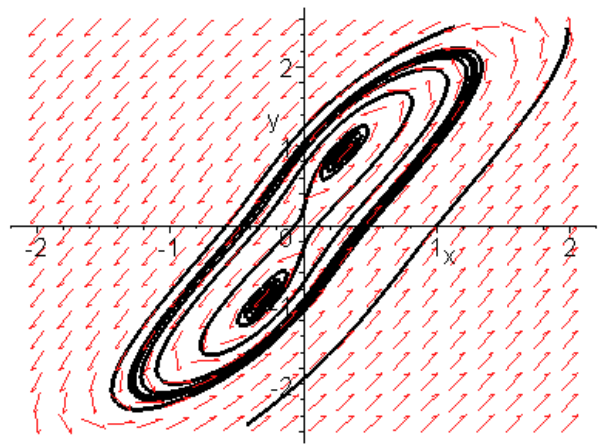

$N<\alpha^{*}=6<\alpha=6.2$

Figure 9. Theorem 5.6: $\varepsilon=-1, N / 2<\delta=3<N=4$.

- $\mathscr{T}_{r}$ does not stay in $\mathscr{2}_{1}$; then $\mathscr{T}_{s}$ is bounded at $-\infty$, and so converges to $M_{\ell}$ at $-\infty$ and spirals around this point, or it has a limit cycle around $M_{\ell}$. Then $\mathscr{T}_{S}$ meets $\mathscr{E}$ at a last point $\left(\ell, Y_{S}(\alpha)\right)$ and $\mathscr{T}_{r}$ meets $\mathscr{E}$ at a first point $\left(\ell, Y_{r}(\alpha)\right)$ such that $Y_{s}(\alpha)-Y_{r}(\alpha)<0$. See Figure 9 , bottom row.

- $\mathscr{T}_{r}$ is homoclinic, which is equivalent to $Y_{s}(\alpha)-Y_{r}(\alpha)=0$. See Figure 9, top right.

Now the function $\alpha \mapsto g(\alpha)=Y_{s}(\alpha)-Y_{r}(\alpha)$ is continuous. If $\alpha_{1}<\eta$, then $g(\eta)$ is defined and $g(\eta)>0$, by Theorem 5.4. If $\eta \leq \alpha_{1}$, we observe that for $\alpha=\alpha_{1}$, the trajectory $\mathscr{T}_{s}$ leaves $2_{1}$, by Theorem 2.18 , because $\alpha_{1}$ is a sink, and does so transversally by Remark 2.1(i). The same holds for $\alpha=\alpha_{1}+\gamma$ for $\gamma$ small enough, by continuity, so $\mathscr{T}_{r}$ stays in $\mathscr{2}_{1}$ and $g\left(\alpha_{1}+\gamma\right)>0$. If $\alpha \geq \alpha^{*}$ (Figure 9 , bottom right), then $M_{\ell}$ is a source or a weak source, by Theorem 2.16; thus $\mathscr{T}_{r}$ cannot converge to $M_{\ell}$. By Theorem 2.19, there exists no cycle in $\mathscr{2}_{1}$ and no homoclinic orbit. By Remark 5.3(i), $\mathscr{T}_{r}$ cannot stay in $\mathscr{2}_{1}$, so $g(\alpha)<0$ for $\alpha^{*} \leq \alpha<\alpha_{2}$. As a 
consequence, there exists at least one $\alpha_{\text {crit }} \in\left(\max \left(\eta, \alpha_{1}\right), \alpha^{*}\right)$ such that $g\left(\alpha_{\text {crit }}\right)=0$. If it is not unique, we can choose the largest one.

(ii) Suppose $\alpha<\alpha^{*}$. The existence and uniqueness of the desired cycle 0 in $\mathscr{2}_{1}$ follows by Theorem 2.16 when $\alpha$ is close to $\alpha^{*}$ (Figure 9, bottom left). In fact, existence holds for any $\alpha \in\left(\alpha_{\text {crit }}, \alpha^{*}\right)$; indeed $g(\alpha)<0$ on this interval, and $\mathscr{T}_{s}$ cannot converge to $M_{\ell}$ at $-\infty$, so it has a limit cycle around $M_{\ell}$ at $-\infty$. Since $M_{\ell}$ is a sink, there exist also trajectories converging to $M_{\ell}$ at $\infty$, with a limit cycle at $-\infty$ contained in 0 . Now $\mathscr{T}_{r}$ does not stay in $2_{1}$ and is bounded at $\infty$, so it has a limit cycle at $\infty$ containing the three stationary points.

(iii) Suppose $\alpha \geq \alpha^{*}$. Then $\mathscr{T}_{s}$ stays in $\mathscr{2}_{1}$, is bounded on $\mathbb{R}$, and converges at $-\infty$ to $M_{\ell}$. At the same time, $\mathscr{T}_{r}$ does not stay in $\mathscr{2}_{1}$ for the same reason as above; thus it has a limit cycle at $\infty$, containing the three stationary points (see Figure 9, bottom right).

(iv) For any $\alpha>\alpha_{\text {crit }}$, apart from $\mathscr{T}_{s}$ and the cycles, all the trajectories have a limit cycle at $\infty$ containing the three stationary points. By Theorem 2.21, all the cycles are contained in a ball $B$ of $\mathbb{R}^{2}$. Take any point $P$ exterior to $B$. By Remark 5.3(ii), $\mathscr{T}_{[P]}$ has a limit cycle at $\infty$ contained in $B$ and cannot have a limit cycle at $-\infty$. Thus $y$ has constant sign near $\ln R_{w}$. By Proposition 2.8, either $R_{w}>0$ or $y$ is defined near $-\infty$ and $\lim _{\tau \rightarrow-\infty} \zeta=L, \lim _{r \rightarrow 0} r^{\alpha} w=L$.

Note. It is an open question whether $\alpha_{\text {crit }}$ is unique. It can be shown that if there exist two critical values $\alpha_{\text {crit }}^{1}>\alpha_{\text {crit }}^{2}$, the first orbit is contained in the second.

When $\delta \leq N / 2$, or equivalently $p \leq P_{2}$, there are no cycles in $\mathbb{R}^{2}$ and we get:

Theorem 5.7. Assume $\varepsilon=1, \delta \leq N / 2$, and $\delta<\alpha$. All regular solutions of $\left(E_{w}\right)$ have constant sign, and $\lim _{r \rightarrow \infty} r^{\delta}|w|=\ell$. All solutions have a finite number of zeros. The function $w(r)=\ell r^{-\delta}$ is a solution. If $\alpha \leq \eta$, Theorem 5.4 applies. If $\eta<\alpha$, all other solutions have at least one zero. There exist solutions satisfying $\lim _{r \rightarrow \infty} r^{\eta} w=c \neq 0$ and having $m$ zeros, for some $m>0$. All other solutions satisfy $\lim _{r \rightarrow \infty} r^{\delta} w= \pm \ell$, and have $m$ or $m+1$ zeros. There exist solutions with $m+1$ zeros.

Proof. (i) By Proposition 2.11, all solutions have a finite number of zeros. Since $\delta \leq N / 2$, the function $W$ defined in (2-21) is nonincreasing. The regular solutions $(y, Y)$ satisfy $\lim _{\tau \rightarrow-\infty} W(\tau)=0$, so $W(\tau) \leq 0$ on $\mathbb{R}$. If $y\left(\tau_{0}\right)=0$ for some real $\tau_{0}$, then $W\left(\tau_{0}\right)=\left|Y\left(\tau_{0}\right)\right|^{p^{\prime}}>0$, and we reach a contradiction. From Propositions 2.8 and 2.11 we obtain $\lim _{\tau \rightarrow \infty} y= \pm \ell$, so $\lim _{r \rightarrow \infty} r^{\delta} w= \pm \ell$.

(ii) Assume $\eta<\alpha$. By Lemma 5.5, $\mathscr{T}_{s}$ does not stay in $\mathscr{2}_{1}$. By Propositions 2.8 and $2.15, \mathscr{T}_{s}$ cannot stay in $2_{4}$, so it intersects the line $y=0$ at points $\left(0, \xi_{1}\right), \ldots$, $\left(0, \xi_{m}\right)$. By Remark 5.3, any trajectory other than $\mathscr{T}_{s}$ converges to $\pm M_{\ell}$. Given 
any $P=(0, \xi)$, with $\xi>\left|\xi_{i}\right|$ for $1 \leq i \leq m$, the trajectory $\mathscr{T}_{[P]}$ cannot intersect $\mathscr{T}_{s}$ or $-\mathscr{T}_{s}$, so $y$ has $m+1$ zeros. Any other solution has $m$ or $m+1$ zeros, because the trajectory does not meet $\mathscr{T}_{r}$ or $-\mathscr{T}_{r}$ or $\mathscr{T}_{[P]}$. Finally, $R_{w}>0$ or $\lim _{r \rightarrow 0} r^{\alpha} w=L \neq 0$.

Note. Theorems 5.4, 5.6 and 5.7 recover, in particular, the results in [Qi and Wang 1999, Theorem 2].

\section{The case $\varepsilon=-1, \alpha \leq \delta$$$
\max (\alpha, N) \leq \delta
$$

Here $(0,0)$ is the only stationary point, and it is a source when $\delta \neq N$. We first suppose $0<\alpha$.

Theorem 6.1. Suppose $\varepsilon=-1, \max (\alpha, N) \leq \delta$ and $0<\alpha$.

(i) Suppose $\alpha \neq N$ or $\alpha \neq \delta$. Then all regular solutions of $\left(E_{w}\right)$ have constant sign and a reduced domain $\left(S_{w}<\infty\right)$. There exist solutions satisfying any one of these characterizations:

(1) $w$ is positive, $\lim _{r \rightarrow 0} r^{\eta} w=c \neq 0$ if $N \geq 2\left(\lim _{r \rightarrow 0} w=a>0, \lim _{r \rightarrow 0} w^{\prime}=\right.$ $b<0$ if $N=1)$, and $\lim _{r \rightarrow \infty} r^{\alpha} w=L \neq 0$ if $\alpha \neq \delta$, or (2-39) holds if $\alpha=\delta$;

(2) $w$ is positive, $\lim _{r \rightarrow 0} r^{\eta} w=c \neq 0$ if $N \geq 2\left(\lim _{r \rightarrow 0} w=a>0, \lim _{r \rightarrow 0} w^{\prime}=\right.$ $b \neq 0$, or $a=0<b$ if $N=1)$, and $S_{w}<\infty$;

(3) $w$ has one zero, $\lim _{r \rightarrow 0} r^{\eta} w=c \neq 0$ if $N \geq 2\left(\lim _{r \rightarrow 0} w=a>0, \lim _{r \rightarrow 0} w^{\prime}=\right.$ $b<0$ if $N=1$ ), and $S_{w}<\infty$.

(ii) Suppose $\alpha=\delta=N$. Then the regular solutions, given by (1-6), have constant sign, with $S_{w}<\infty$. For any $k \in \mathbb{R}, w(r)=k r^{-N}$ is a solution. Moreover there exist positive solutions such that $\lim _{r \rightarrow 0} r^{N} w=c>0$ and $S_{w}<\infty$, and solutions with one zero, such that $\lim _{r \rightarrow 0} r^{N} w=c>0$ and $S_{w}<\infty$.

Up to symmetry, all solutions are as above.

Proof. (i) Here $\alpha \neq N$ or $\alpha \neq \delta$ (Figure 10, left). Since $\alpha>0$, Propositions 2.5, 2.7 and 2.14 imply that $y>0$ and $S_{w}<\infty$ for $\mathscr{T}_{r}$; and any solution $y$ has at most one zero, and $y, Y$ are monotone near $-\infty$ and near $\ln S_{w}$. By Proposition 2.8, any trajectory $\mathscr{T}$ converges to $(0,0)$ at $-\infty$; and apart from $\mathscr{T}_{r}$, such a trajectory is tangent to the axis $y=0$. Now suppose $y>0$ near $-\infty$. If $N \geq 2$, then $\mathscr{T}$ starts in $2_{1}$, since $\lim _{\tau \rightarrow-\infty} \zeta=\eta>0$; if $N=1$, then $\lim _{r \rightarrow 0} w=a \geq 0$ and $\lim _{r \rightarrow 0} w^{\prime}=b$, and $\mathcal{T}$ starts in $2_{1}$ if $b<0$ and in $2_{4}$ if $b>0$ (in particular when $a=0$ ).

For any $P=(\varphi, 0)$ with $\varphi>0$, the trajectory $\mathscr{T}_{[P]}$ satisfies $y>0$ on $\mathbb{R}$, and by Remark 2.1(i), it stays in $2_{4}$ after $P$, because it cannot meet $\mathscr{T}_{r}$ (hence $S_{w}<\infty$ ); also it stays in $2_{1}$ before $P$, so $w$ is of type (2). In the same way for any $P=(0, \xi)$ 


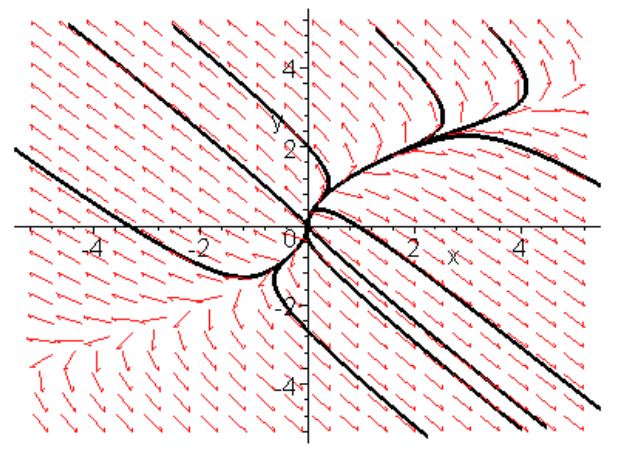

$N=2<\alpha=2.5<\delta=3$

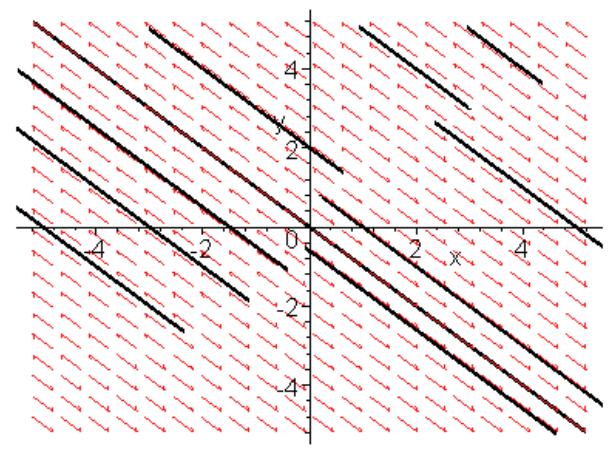

$\alpha=\delta=N=3$

Figure 10. Theorem 6.1: $\varepsilon=-1$.

with $\xi>0$, the trajectory $\mathscr{T}_{[P]}$ stays in $\mathscr{2}_{2}$ after $P$, since it cannot meet $-\mathscr{T}_{r}$ (hence $\left.S_{w}<\infty\right)$, and it stays in $2_{1}$ before $P$, so $w$ is of type (3).

Next consider the sets

$$
\begin{aligned}
& \mathscr{A}=\left\{P \in \mathscr{Q}_{1}: \mathscr{T}_{[P]} \cap\{(\varphi, 0): \varphi>0\} \neq \varnothing\right\}, \\
& \mathscr{B}=\left\{P \in \mathscr{2}_{1}: \mathscr{T}_{[P]} \cap\{(0, \xi): \xi>0\} \neq \varnothing\right\} .
\end{aligned}
$$

From the previous discussion we know they are nonempty and open, so $\mathscr{A} \cup \mathscr{B} \neq \mathscr{2}_{1}$. There exists a trajectory $\mathscr{T}_{1}$ starting at $(0,0)$ and staying in $2_{1}$. By Proposition 2.8, necessarily $\lim _{\tau \rightarrow \infty} y=\infty$ and $\lim _{\tau \rightarrow \infty} \zeta=\alpha>0$, so $w$ is of type (1) by Proposition 2.9.

Finally we describe all other trajectories $\mathscr{T}_{[P]}$ with one point $P$ in the domain $\mathscr{R}$ above $\mathscr{T}_{r} \cup\left(-\mathscr{T}_{r}\right)$. If $P$ is in the domain delimited by $\mathscr{T}_{r}, \mathscr{T}_{1}$, then $w$ is still of the type (2). If $P$ is in the domain delimited by $-\mathscr{T}_{r}, \mathscr{T}_{1}$, then either $y$ has a zero and $w$ is of type (3), or $N=1, y<0$ and $-w$ is of type (2). Up to a symmetry, all the solutions have been obtained.

(ii) Here $\alpha=\delta=N$ (Figure 10, right). Since $\alpha=N$ equation (1-5) holds, and the regular solutions relative to $C=0$ are given by (1-6). Since $\delta=N,(1-5)$ is equivalent to $y+Y \equiv C$, from (2-12). For any $k \in \mathbb{R},(y, Y) \equiv P_{k}=\left(k,|N k|^{p-2} N k\right)$ is a solution of system $(S)$, located on the curve $M$, thus $w(r)=k r^{-N}$ is a solution of $\left(E_{w}\right)$. Any solution has at most one zero, by Proposition 2.5. From Propositions 2.8, and 2.10, any other trajectory converges to a point $P_{k} \in \mathcal{M}$ at $\infty$, and $S_{w}<\infty$. There exists trajectories such that $y$ has constant sign, and other ones such that $y$ has one zero. All solutions have been obtained.

Next we suppose $\alpha<0$, and distinguish the cases $N \geq 2$ and $N=1$.

Theorem 6.2. Suppose $\varepsilon=-1$ and $\alpha<0<2 \leq N \leq \delta$. Then any solution of $\left(E_{w}\right)$ has a finite number of zeros. Regular solutions have at least one zero, and 
precisely one if $-p^{\prime} \leq \alpha$. Any solution has at least one zero, and any nonregular solution satisfies $\lim _{r \rightarrow 0} r^{\eta} w=c \neq 0$.

If $-p^{\prime}<\alpha$, all regular solutions have a reduced domain $\left(S_{w}<\infty\right)$, and they fall into the following types, all of which occur:

(1) solutions with two zeros and $S_{w}<\infty$;

(2) solutions with one zero and $\lim _{r \rightarrow \infty} r^{\alpha} w=L \neq 0$;

(3) solutions with one zero and $S_{w}<\infty$.

If $\alpha=-p^{\prime}$, all regular solutions satisfy $\lim _{r \rightarrow \infty} r^{\alpha} w=L \neq 0$. The other solutions are of type (1).

Proof. By Proposition 2.8, any trajectory converges necessarily to $(0,0)$ at $-\infty$, and apart from $\mathscr{T}_{r}$, it is tangent to the axis $y=0$. Any solution $y$ has a finite number of zeros, and $y$ is monotone near $-\infty$, and near $S_{w}$ (finite or not), by Propositions 2.7 and 2.11, since $\delta>N / 2$. Either $S_{w}<\infty$, so $\lim _{\tau \rightarrow \ln } S_{w} Y / y=-1$, or $S_{w}=\infty$ and $\lim _{\tau \rightarrow \infty} \zeta=\alpha<0$. In any case $(y, Y)$ is in $2_{2}$ or $2_{4}$ for large $\tau$. By Proposition 2.14, $\mathscr{T}_{r}$ has at least one zero, and starts in $\mathscr{2}_{1}$. Since $N \geq 2$, any trajectory $\mathscr{T} \neq \pm \mathscr{T}_{r}$ satisfies $\lim _{\tau \rightarrow-\infty} \zeta=\eta>0$. Thus it starts in $\mathscr{2}_{1}$ (or $\mathscr{2}_{3}$ ), and has at least one zero. Any trajectory $\mathscr{T}$ starting in $\mathscr{2}_{1}$ enters $\mathscr{2}_{2}$, by Remark 2.1(i). And $y^{\prime}=\delta y-Y^{1 /(p-1)}$, so $y$ decreases as long as $\mathcal{T}$ stays in $2_{2}$. Then either $\mathscr{T}$ enters $2_{3}$, hence also $2_{4}$, and $y$ has at least two zeros; or it stays in $2_{2}$, and either $S_{w}<\infty$ and $\lim _{\tau \rightarrow \ln S_{w}} Y / y=-1$, or $S_{w}=\infty$ and $\lim _{\tau \rightarrow \infty} \zeta=\alpha$.

(i) Suppose $-p^{\prime}<\alpha$ (Figure 11, left). Then $\mathscr{T}_{r}$ has precisely one zero, by Proposition 2.14, thus it stays in $2_{2}$, and $S_{w}<\infty, \lim _{\tau \rightarrow \ln } S_{w} Y / y=-1$. Any other solution has at most two zeros, because the trajectory does not meet $\pm \mathscr{T}_{r}$. Recall that the function $Y_{\alpha}$ defined by (2-3) with $d=\alpha$ has only minimal points on the

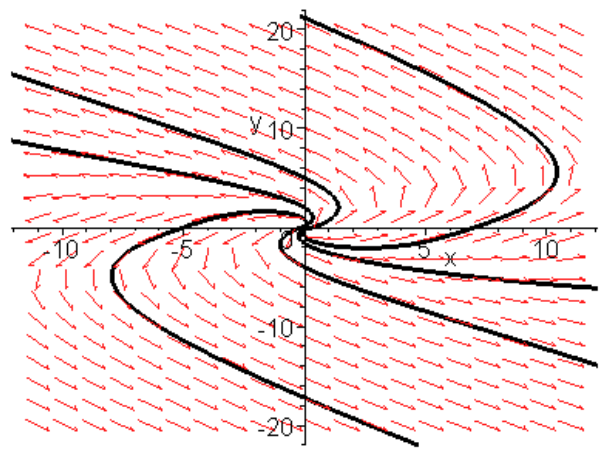

$-p^{\prime}=-3<\alpha=-2.5<0<N$

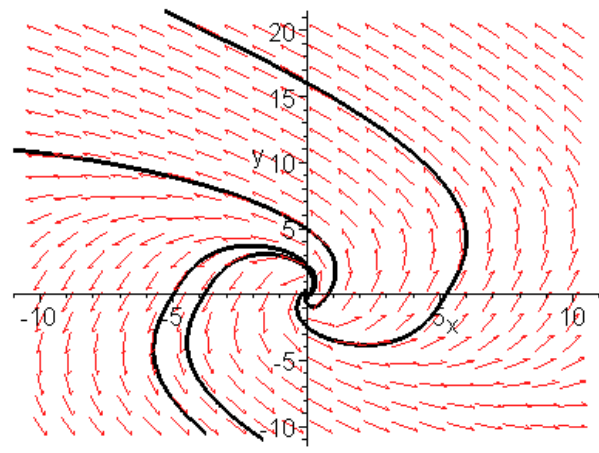

$-p^{\prime}=-3=\alpha<0<N$

Figure 11. Theorem 6.2: $\varepsilon=-1, N=2<\delta=3$. 
sets where it is positive, by Remark 2.6. By Proposition 2.14, $\mathscr{T}_{r}$ satisfies

$$
Y_{\alpha}^{\prime}=-(p-1)(\eta-\alpha) Y_{\alpha}+e^{(p-(2-p) \alpha) \tau}\left(Y_{\alpha}^{1 /(p-1)}-\alpha y_{\alpha}\right)>0,
$$

which is equivalent to

$$
Y^{1 /(p-1)}-(p-1)(\eta-\alpha) Y>\alpha y .
$$

$\mathscr{T}_{r}$ stays strictly to the right of the curve

$$
\mathcal{N}_{\alpha}=\left\{(y, Y) \in \mathbb{R} \times(0, \infty): \alpha y=Y^{1 /(p-1)}-(p-1)(\eta-\alpha) Y\right\},
$$

which intersects the axis $y=0$ at the points $(0,0)$ and $(0,(p-1)(\eta-\alpha))$.

For $\bar{P}=(\varphi, 0)$ with $\varphi<0$, the trajectory $\mathscr{T}_{[\bar{P}]}$ enters $2_{3}$ after $\bar{P}$, by Remark 2.1(i); the solution passing through $\bar{P}$ at $\tau=0$ satisfies $Y_{\alpha}(0)=0$ (so $Y_{\alpha}$ stays positive for $\tau<0$ ) and $Y_{\alpha}^{\prime}(\tau)<0$, since $Y_{\alpha}$ has no maximal point. Thus $\mathscr{T}_{[\bar{P}]}$ stays in $\mathscr{2}_{1} \cup \mathscr{2}_{2}$ before $P$, to the left of $\mathcal{N}_{\alpha}$, and starts and $(0,0)$ in $\mathscr{2}_{1}$ and ends up in 24 . Hence $y$ has two zeros. If $S_{w}=\infty$ then $\lim _{\tau \rightarrow \infty}|y|=\infty$ and $\lim _{\tau \rightarrow \infty} \zeta=\alpha<0$; this is impossible, because $\mathscr{T}_{[\bar{P}]}$ does not meet $-\mathscr{T}_{r}$. Thus $S_{w}<\infty$, and $w$ is of type (1).

Next consider $\mathscr{T}_{[P]}$, for $P=(\varphi, \xi) \in \mathcal{N}_{\alpha}$, with $\varphi \leq 0$. The solution going through $P$ at $\tau=0$ satisfies $Y_{\alpha}^{\prime}(0)=0, Y_{\alpha}(0)>0$, and 0 is a minimal point; hence $Y_{\alpha}^{\prime \prime}(0)>0$. Indeed, if $Y_{\alpha}^{\prime \prime}(0)=0$, then $Y_{\alpha}$ is constant on $\mathbb{R}$ by uniqueness; by (2-6), in turn, we have $Y_{\alpha} \equiv 0$ (since $\alpha \neq-p^{\prime}$ ); but this is false. Therefore $Y_{\alpha}^{\prime}(\tau)>0$ for $\tau>0$ and $Y_{\alpha}^{\prime}(\tau)<0$ for $\tau<0$. Thus $\mathscr{T}_{[P]}$ stays in $\mathscr{2}_{1} \cup 2_{2}$, to the right of $\mathcal{N}_{\alpha}$ after $P$, with $y<0$ by Remark 2.1(i); it stays to the left of $\mathcal{N}_{\alpha}$ before $P$, and converges to $(0,0)$ at $-\infty$ in $2_{1}$. Suppose that $S_{w}=\infty$. Then $\lim _{\tau \rightarrow \infty}|y|=\infty, \lim _{\tau \rightarrow \infty} \zeta=\alpha$, and $\lim _{\tau \rightarrow \infty} y_{\alpha}=L<0$ by Proposition 2.9; thus $\lim _{\tau \rightarrow \infty} Y_{\alpha}=(\alpha L)^{p-1}$. As in Proposition 2.14, one finds that $Y_{\alpha}^{\prime \prime}(\tau)>0$ for any $\tau>0$, which is impossible. Thus $\mathscr{T}_{[P]}$ satisfies $S_{w}<\infty$, showing that $\lim _{\tau \rightarrow \ln S_{w}} Y / y=-1$. The corresponding $w$ is of type (3).

Finally, let $\mathscr{R}$ be the domain of $\mathscr{2}_{1} \cup \mathscr{2}_{2}$ delimited by $\mathscr{T}_{r}$ and containing $\mathcal{N}_{\alpha}$, and define the sets

$$
\begin{aligned}
& \mathscr{A}=\left\{P \in \mathscr{R}: \mathscr{T}_{[P]} \cap\{(\varphi, 0): \varphi<0\} \neq \varnothing\right\}, \\
& \mathscr{B}=\left\{P \in \mathscr{R}: \mathscr{T}_{[P]} \cap \mathcal{N}_{\alpha} \neq \varnothing\right\},
\end{aligned}
$$

corresponding to trajectories of type (1) or (3). These sets are nonempty and open, because here again the intersection with $\mathcal{N}_{\alpha}$ is transverse (recall that $\alpha \neq-p^{\prime}$ ). Thus $\mathscr{A} \cup \mathscr{B} \neq \mathscr{R}$. There exists a trajectory in $\mathscr{R}$ disjoint from $\mathcal{N}_{\alpha}$, starting at $(0,0)$ in $2_{1}$ and ending up in $2_{2}$. It cannot satisfy $\lim _{\tau \rightarrow \ln } S_{w} Y / y=-1$, so $S_{w}=\infty$ and $\lim _{\tau \rightarrow \infty} \zeta=\alpha$. Hence $w$ is of type (2). 
(ii) Suppose $\alpha=-p^{\prime}$ (Figure 11, right). The regular solutions are given by (1-8), they have one zero, but $S_{w}=\infty$ and $\lim _{\tau \rightarrow \infty} \zeta=\alpha$. They satisfy $Y_{-p^{\prime}} \equiv C$, thus $Y_{-p^{\prime}}^{\prime} \equiv 0$, thus $\mathscr{T}_{r}=M_{-p^{\prime}}$. Consider $\mathscr{T}_{[\bar{P}]}$; the solution passing through $\bar{P}$ at $\tau=0$ satisfies and $Y_{-p^{\prime}}(0)=0$, thus $Y_{-p^{\prime}}$ stays negative for $\tau>0$ and $Y_{-p^{\prime}}^{\prime}<0$. Suppose that $S_{w}=\infty$, then $\lim _{\tau \rightarrow \infty} y_{\alpha}=L>0, \lim _{\tau \rightarrow \infty} Y_{\alpha}=-(|\alpha| L)^{p-1}$. But as in (2-46), $Y_{\alpha}^{\prime \prime}(\tau)<0$ for any $\tau>0$, which leads to a contradiction. Thus $S_{w}<\infty$, and $w$ is of type (1). Finally suppose that there exists a trajectory $\mathcal{T} \neq \mathscr{T}_{r}$ staying in $2_{1} \cup 2_{2}$. Then $Y_{\alpha}>0, \lim _{\tau \rightarrow \infty} Y_{\alpha}=0$, and it cannot meet $\mathscr{T}_{r}$, thus $S_{w}=\infty$, and $\lim _{\tau \rightarrow-\infty} Y_{\alpha}=\infty, \lim _{\tau \rightarrow \infty} Y_{\alpha}=C>0$. As in Proposition 2.14, it is impossible. Thus there does not exist solution of type (2) or (3).

Theorem 6.3. Suppose $\varepsilon=-1$ and $\alpha<0<N=1<\delta$. Then any solution of $\left(E_{w}\right)$ has still a finite number of zeros. Regular solutions have at least one zero, and precisely one if $-p^{\prime} \leq \alpha$.

If $-1<\alpha<0$, all regular solutions have a reduced domain $\left(S_{w}<\infty\right)$. Moreover:

(1) the solutions with $\lim _{r \rightarrow 0} w=a>0$ and $\lim _{r \rightarrow 0} w^{\prime}=b<0$ have one zero and $S_{w}<\infty$;

(2) the solutions with $\lim _{r \rightarrow 0} w=0$ and $\lim _{r \rightarrow 0} w^{\prime}=b>0$ are positive and $S_{w}<\infty$

(3) there exist solutions with one zero and $\lim _{r \rightarrow 0} w=a>0, \lim _{r \rightarrow 0} w^{\prime}=b>0$ and $S_{w}<\infty$;

(4) there exist positive solutions with $\lim _{r \rightarrow 0} w=a>0, \lim _{r \rightarrow 0} w^{\prime}=b>0$ and $S_{w}<\infty$

(5) for any $a>0$ there exists $b>0$ such that $w$ is positive and $\lim _{r \rightarrow \infty} r^{\alpha} w=$ $L \neq 0$.

If $\alpha=-1$, for any $b>0, w \equiv b r$ is a solution. The other solutions such that $\lim _{r \rightarrow 0} w \neq 0$ have one zero, and satisfy $S_{w}<\infty$.

If $-p^{\prime}<\alpha<-1$, then

(6) there exist solutions with one zero, with $\lim _{r \rightarrow 0} w=a>0, \lim _{r \rightarrow 0} w^{\prime}=b<0$, and $S_{w}<\infty$

(7) the solutions with $\lim _{r \rightarrow 0} w=0$ and $\lim _{r \rightarrow 0} w^{\prime}=b>0$ have one zero and $S_{w}<\infty$

(8) there exist solutions with one zero, with $\lim _{r \rightarrow 0} w=a>0, \lim _{r \rightarrow 0} w^{\prime}=b>0$ and $S_{w}<\infty$;

(9) there exist solutions with $\lim _{r \rightarrow 0} w=a>0, \lim _{r \rightarrow 0} w^{\prime}=b<0$, with two zeros and $S_{w}<\infty$; 

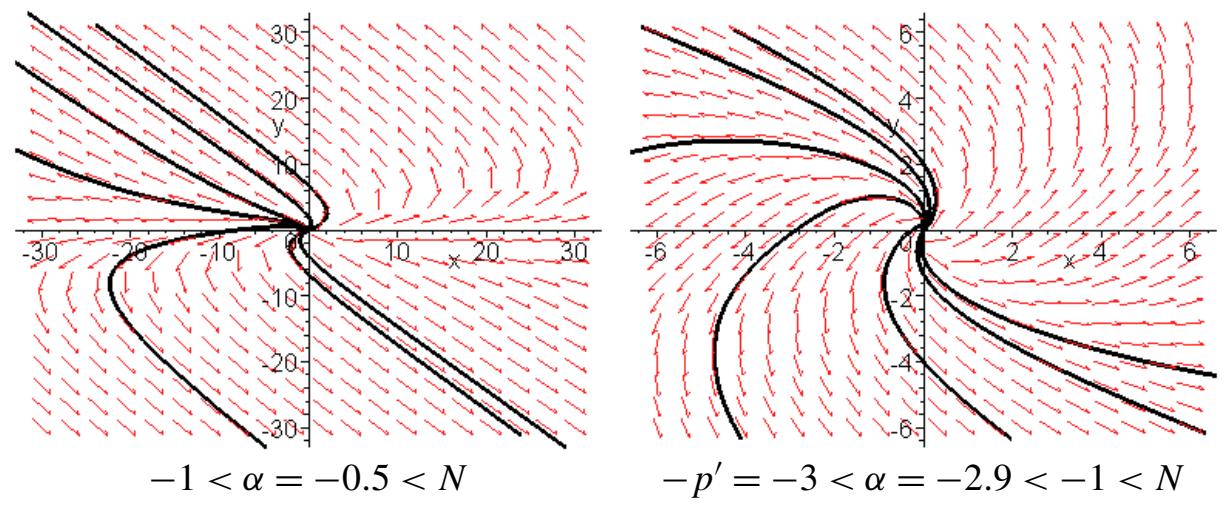

Figure 12. Theorem 6.3: $\varepsilon=-1, N=1<\delta=3$.

(10) for any $a>0$ there exists $b>0$ and a solution with $\lim _{r \rightarrow 0} w=a>0$, $\lim _{r \rightarrow 0} w^{\prime}=b<0$, with one zero and $\lim _{r \rightarrow \infty} r^{\alpha} w=L \neq 0$.

Proof. The case $N=1$ is still the more complex one, since some trajectories start in $2_{2}$ (or 24 ), corresponding to the solutions such that $\lim _{r \rightarrow 0} w=a$ and $\lim _{r \rightarrow 0} w^{\prime}=b$, with $b \neq 0, a b \geq 0$. Any solution has still a finite number of zeros, by Proposition 2.11 .

(i) Suppose $-1<\alpha<0$ (Figure 12, left). By Proposition 2.5, any solution has at most one zero, so regular solutions have precisely one zero. Thus $\mathscr{T}_{r}$ meets the axis $y=0$ at some point $\left(0, \xi_{r}\right)$.

Consider the trajectory $\mathscr{T}_{s}$ such that $\lim _{r \rightarrow 0} w=0$ and $\lim _{r \rightarrow 0} w^{\prime}=b<0$ (which means $\left.\lim _{\tau \rightarrow-\infty} \zeta=\eta=-1\right)$, starting from $(0,0)$ in $2_{2}$, so $w<0$ near 0 . For any $d \in(-1, \alpha)$, the function $y_{d}$ satisfies $y_{d}(\tau)=b e^{(d+1) \tau}(1+o(1))$ near $-\infty$, so $\lim _{\tau \rightarrow-\infty} y_{d}=0$. Then $y_{d}$ has no zeros, because $\left|y_{d}\right|$ has no maximal point, by (2-14); thus $\mathscr{T}_{s}$ stays in $2_{2}$. If $\mathscr{T}_{s}$ satisfies $S_{w}=\infty$, then $\lim _{\tau \rightarrow \infty} y_{\alpha}=L<0$, so $\lim _{\tau \rightarrow \infty} y_{d}=0$, which is impossible; thus $w$ is of type (2). The domain is reduced since $\mathscr{T}_{r}$ cannot meet $\mathscr{T}_{s}$.

For $\bar{P}=(\varphi, 0)$ with $\varphi<0$, the trajectory $\mathscr{T}_{[\bar{P}]}$ does not meet $\mathscr{T}_{s}$, thus converges to $(0,0)$ at $-\infty$ in $2_{2}$; then $\lim _{r \rightarrow 0}(-w)=a>0$ and $\lim _{r \rightarrow 0}(-w)^{\prime}=b>0$, and $\mathscr{T}_{[\bar{P}]}$ ends up in $2_{4}$; thus $y$ has one zero and $-w$ is of type (3).

For $P=(0, \xi)$, with $\xi \in\left(0, \xi_{r}\right), \mathscr{T}_{[P]}$ has one zero and converges to $(0,0)$ at $-\infty$ in $2_{1}$; hence $\lim _{r \rightarrow 0} w=a>0$ and $\lim _{r \rightarrow 0} w^{\prime}=b<0$. The domain is reduced since $\mathscr{T}_{[P]}$ and $\mathscr{T}_{s}$ do not meet. Thus $w$ is of type (1). Conversely, any solution such that $\lim _{r \rightarrow 0} w=a>0$ and $\lim _{r \rightarrow 0} w^{\prime}=b<0$ has one zero and satisfies $S_{w}<\infty$.

Next consider a trajectory $\mathscr{T}$ such that $\lim _{r \rightarrow 0}(-w)=a>0$ and $\lim _{r \rightarrow 0}\left(-w^{\prime}\right)=$ $b>0$; that is, $\mathscr{T}$ starts in $\mathscr{2}_{2}$ below $\mathscr{T}_{s}$. Then $\zeta(\tau)=-(b / a) e^{\tau}(1+o(1)$ near $-\infty$, 
so $\lim _{\tau \rightarrow-\infty} \zeta=0$. If $\zeta$ has an extremal point $\theta$, we have

$$
(p-1) \zeta^{\prime \prime}(\theta)=(2-p)(\zeta-\alpha)(\delta-\zeta)|\zeta y|^{2-p},
$$

by (2-18); thus $\theta$ is a minimal point if $\zeta(\theta)>\alpha$, and maximal if $\zeta(\theta)<\alpha$. (Equality is impossible since it would require $\zeta \equiv \alpha$.) Thus either $\zeta$ has a first zero $\tau_{1}$ and $\alpha<\zeta(\tau)<0$ for $\tau<\tau_{1}$, and $\mathscr{T}$ is one of the $\mathscr{T}_{[\bar{P}]}$; or $\zeta$ remains negative, in which case if $S_{w}=\infty$, then $\lim _{\tau \rightarrow \infty} \zeta=\alpha$, so $\zeta$ is necessarily decreasing, and $\alpha<\zeta(\tau)<0$ for any $\tau$. In both cases, $\mathcal{T}$ stays below the curve

$$
\mathcal{M}^{\prime}=\left\{(y, Y) \in \mathbb{R} \times(0, \infty): \alpha y=Y^{1 /(p-1)}\right\},
$$

as long as it is in $2_{2}$. Hence, for any $P \in 2_{2}$ such that $P$ is on or above $\mathcal{M}^{\prime}$, the trajectory $\mathscr{T}_{[P]}$ satisfies $S_{w}<\infty$; in particular on finds again $\mathscr{T}_{s}$. For any $P$ between $\mathcal{M}^{\prime}$ and $\mathscr{T}_{s}$, the solution has constant sign, $\mathscr{T}_{[P]}$ converges to $(0,0)$ at $-\infty$ and $\lim _{r \rightarrow 0}(-w)=a>0$ and $\lim _{r \rightarrow 0}\left(-w^{\prime}\right)=b>0$, and $\lim _{\tau \rightarrow \ln S_{w}} Y / y=-1$, so $\mathscr{T}_{[P]}$ meets $\mu_{\alpha}$. Thus $-w$ is of type (4).

Finally, let $\mathscr{R}_{1}$ be the domain of $\mathscr{2}_{2}$ delimited by $\mathscr{T}_{s}$ and the axis $Y=0$, and set

$$
\begin{aligned}
& \mathscr{A}_{1}=\left\{P \in \mathscr{R}_{1}: \mathscr{T}_{[P]} \cap\{(\varphi, 0): \varphi<0\} \neq \varnothing\right\}, \\
& \mathscr{R}_{1}=\left\{P \in \mathscr{R}_{1}: \mathscr{T}_{[P]} \cap \mathcal{N}_{\alpha} \neq \varnothing\right\} .
\end{aligned}
$$

These sets are open, since the intersection is transverse (recall that $\alpha \neq-1$ ). They are also nonempty, so $\mathscr{A}_{1} \cup \mathscr{B}_{1} \neq \mathscr{R}_{1}$, and there exists a trajectory such that $y$ is defined on $\mathbb{R}$ and $\lim _{\tau \rightarrow \infty} \zeta=\alpha$. By scaling, we can find for any $a>0$ at least one $b$ such that the corresponding $w$ has constant sign and $\lim _{r \rightarrow \infty} r^{\alpha} w=L \neq 0$; thus $|w|$ is of type (5).

(ii) Suppose $\alpha=-1$. Then $\mathscr{T}_{s}$ is given explicitly by $w \equiv b r$, so $Y \equiv-y^{p-1}$, or equivalently $Y_{-1} \equiv b$; hence $\mathscr{T}_{s}=\mathcal{N}_{-1}$. For any other solution, one finds $Y_{-1}^{\prime \prime}=$ $Y_{-1}^{\prime}\left(1+e^{2 \tau}\left|Y_{-1}\right|^{(2-p) /(p-1)}\right)$, so $Y_{-1}$ is strictly monotone, by uniqueness, and $Y_{-1}^{\prime \prime}$ has the sign of $Y_{-1}^{\prime}$. Any trajectory such that $\lim _{r \rightarrow 0} w=a>0$ and $\lim _{r \rightarrow 0} w^{\prime}=$ $b<0$, starting in $2_{1}$, satisfies $Y_{-1}^{\prime}>0$, and $Y_{-1}$ is convex. Thus $Y_{-1}$ cannot have a finite limit, $S_{w}<\infty$, and the trajectory ends up in $2_{2}$, so $y$ has a zero. Any trajectory such that $\lim _{r \rightarrow 0}(-w)=a>0$ and $\lim _{r \rightarrow 0}(-w)^{\prime}=b>0$, starting in $2_{2}$, satisfies $Y_{-1}^{\prime}<0$, so $Y_{-1}$ has a zero and the trajectory ends up in $2_{4}$. Hence, apart from $\mathscr{T}_{s}$, all trajectories satisfy $S_{w}<\infty$, and $y$ has one zero.

(iii) Suppose $-p^{\prime}<\alpha<-1$ (Figure 12, right). Then $\mathscr{T}_{r}$ starts in $\mathscr{2}_{1}, y$ has one zero from Proposition 2.14, and $\mathscr{T}_{r}$ ends up in $2_{2}$, with $S_{w}<\infty$. Any solution has at most two zeros.

Consider $\mathscr{T}_{s}$ : we claim that it cannot stay in $2_{2}$. Suppose that it stays in it, thus $y<0<Y$. Then $\zeta<0$, and $\lim _{\tau \rightarrow-\infty} \zeta=\eta=-1$, and $\zeta$ is monotone near $-\infty$; if $\zeta^{\prime} \leq 0$, then $\zeta \leq-1$ near $-\infty$, and we reach a contradiction from (2-9). Then 
$\zeta^{\prime} \geq 0$ near $-\infty$; but any extremal point of $\zeta$ is a minimal point by (2-18). Hence $\zeta$ remains increasing, is defined on $\mathbb{R}$ and has a limit $\lambda \in[-1,0]$; but $\lambda=\alpha$, by Proposition 2.8, again leading to a contradiction. Therefore $\mathscr{T}_{s}$ enters $\mathscr{2}_{3}$ at some point $\left(\varphi_{s}, 0\right)$ with $\varphi_{s}<0$, then enters $2_{4}$, and $y$ has precisely one zero; and $w$ is of type (7).

Any solution such that $\lim _{r \rightarrow 0}(-w)=a>0$ and $\lim _{r \rightarrow 0}(-w)^{\prime}=b>0$ also has one zero, since its trajectory stays under $\mathscr{T}_{s}$ in $2_{2}$; thus $w$ is of type (8).

As in the case $N \geq 2$, for any $P=(\varphi, \xi) \in \mathcal{N}_{\alpha}$ with $\varphi \leq 0, \mathscr{T}_{[P]}$ stays in $\mathscr{2}_{1} \cup \mathscr{2}_{2}$ and $S_{w}<\infty$. In particular for $P_{0}=\left(0, \xi_{0}\right)$, where $\xi_{0}=((p-1)(-1-\alpha))^{(p-1) /(2-p)}$, the trajectory $\mathscr{T}_{\left[P_{0}\right]}$ starts from $2_{1}$, so $\lim _{r \rightarrow 0} w=a>0, \lim _{r \rightarrow 0} w^{\prime}=b_{0}(a)>0$; also $w$ has one zero, and $S_{w}<\infty$. Thus $w$ is of type (6).

The sets $\mathscr{A}, \mathscr{B}$ defined as in (6-3) are still open in this case, and $\mathscr{B}$ contains $\mathscr{T}_{\left[P_{0}\right]}$. Also, $\mathscr{A}$ contains $\mathscr{T}_{s}$; hence $\mathscr{A}$ contains any $\mathscr{T}_{[P]}$, where $P=(\varphi, 0)$ with $\varphi<\varphi_{s}$. Such a trajectory satisfies $\lim _{r \rightarrow 0} w=a>0$ and $\lim _{r \rightarrow 0} w^{\prime}=b<0$, and $w$ is of type (9). Moreover $\mathscr{A} \cup \mathscr{B} \neq \mathscr{R}$; thus for any $a>0$ there exists $b<0$ such that the corresponding $w$ has one zero and $\lim _{r \rightarrow \infty} r^{\alpha} w=L \neq 0$, so $w$ is of type (10).

$$
\alpha<\delta<N
$$

As in the case $\varepsilon=1, \delta<\min (\alpha, N)$ of page 246, here two kinds of periodic trajectories can appear, and the study is delicate. Here also $N \geq 2$, and we still have three stationary points, and $(0,0)$ is a saddle point. $M_{\ell}$ is a source if $N / 2 \leq \delta$ or $\delta<N / 2$ and $\alpha^{*}<\alpha$, and a sink if $\delta<N / 2$ and $\alpha<\alpha^{*}$; notice that $\alpha^{*}<-p^{\prime}<0$, by (2-32). Also $M_{\ell}$ is a node whenever $\alpha \leq \alpha_{1}$ or $\alpha_{2} \leq \alpha$, where $\alpha_{1}, \alpha_{2}$ are defined in (2-48), and $\alpha_{2}$ can be greater or less than $-p^{\prime}$. We begin with the simplest case.

Theorem 6.4. Assume $\varepsilon=-1$ and $0<\alpha<\delta<N$. All regular solutions have constant sign and a reduced domain $\left(S_{w}<\infty\right)$. The function $w \equiv \ell r^{-\delta}$ is a solution. There exist solutions satisfying any one of these characterizations:

(1) $w$ is positive, $\lim _{r \rightarrow 0} r^{\delta} w=\ell$ and $S_{w}<\infty$;

(2) $w$ has one zero, $\lim _{r \rightarrow 0} r^{\delta} w=\ell$ and $S_{w}<\infty$;

(3) $w$ is positive, $\lim _{r \rightarrow 0} r^{\delta} w=\ell$ and $\lim _{r \rightarrow \infty} r^{\eta} w=c>0$;

(4) $w$ is positive, $\lim _{r \rightarrow 0} r^{\delta} w=\ell$ and $\lim _{r \rightarrow \infty} r^{\alpha} w=L>0$.

Up to symmetry, all solutions are as above.

Proof. Since $\alpha>0$, regular solutions have constant sign and satisfy $S_{w}<\infty$, by Propositions 2.5 and 2.14. Here $\mathscr{T}_{r}$ starts in $2_{4}$ and stays in it, by Remark 2.3 (Figure 13). Any solution has at most one zero by Proposition 2.5. The point $M_{\ell}$ is a source, and a node point, by Remark 2.17, and $0<\lambda_{1}<\delta<\lambda_{2}$. The eigenvectors $u_{1}=\left(v(\alpha), \lambda_{1}-\delta\right)$ and $u_{2}=\left(-v(\alpha), \delta-\lambda_{2}\right)$ form a positively oriented basis, where 
now $v(\alpha)<0$; thus $u_{1}$ points toward $2_{3}$ and $u_{2}$ toward $2_{4}$. There are two particular trajectories $\mathscr{T}_{1}, \mathscr{T}_{2}$ starting from $M_{\ell}$ at $-\infty$, with respective tangent vectors $u_{2}$ and $-u_{2}$. All other trajectories $\mathscr{T}$ approaching $M_{\ell}$ at $-\infty$ do so along $u_{1}$; and $y$ is monotone at the extremities, by Proposition 2.7, since $\mathscr{T}$ cannot meet $\mathscr{T}_{1}, \mathscr{T}_{2}$.

First consider $\mathscr{T}_{1}$. The function $y$ is nondecreasing near $-\infty$ and remains so as long as $\mathscr{T}_{1}$ stays in $2_{1}$; indeed, $Y$ is nonincreasing near $-\infty$, so $Y(\tau)<(\delta \ell)^{p-1}$. If $y$ has a maximal point $\tau$, then $y(\tau)>\ell$ by $(2-16)$, and $Y^{1 /(p-1)}=\delta y$; hence $Y(\tau)>(\delta \ell)^{p-1}$, so $Y$ has a minimal point $\tau_{1}$ in $2_{1}$; therefore $Y\left(\tau_{1}\right)<(\delta \ell)^{p-1}$ by $\left(E_{Y}\right)$; and $Y^{\prime}\left(\tau_{1}\right)=0$, so $\alpha \ell<\alpha y\left(\tau_{1}\right)<(N-\delta) \alpha Y\left(\tau_{1}\right) /(\delta-\alpha)$, a contradiction. If $\mathscr{T}_{1}$ stays in $2_{1}$, then $\lim _{\tau \rightarrow-\infty} \zeta=\alpha>0$ by Proposition 2.8, which is also contradictory. Thus $\mathscr{T}_{1}$ enters $\mathscr{2}_{4}$ at some point $\left(\varphi_{1}, 0\right)$ and stays in it; $S_{w}<\infty$ because $\mathscr{T}_{1}$ and $\mathscr{T}_{r}$ don't meet, so $w$ is of type (1).

Next consider $\mathscr{T}_{2}$. Near $-\infty$, the function $Y$ is nondecreasing, and $y$ is nonincreasing; $y$ is monotone as long as $y>0$ : if there existed a minimal point $\tau$, we would have $y(\tau)>\ell$ by (2-16). Also $Y$ is nondecreasing as long as $Y>0$ : if $Y$ has a maximal point $\tau$, then $Y(\tau)>(\delta \ell)^{p-1}$ by $\left(E_{Y}\right)$; and

$$
\alpha \ell>\alpha y(\tau)>(N-\delta) \alpha Y(\tau) /(\delta-\alpha),
$$

which is again impossible. Thus $\mathscr{T}_{2}$ cannot stay in $\mathscr{2}_{1}$; it enters $\mathscr{2}_{2}$ at some point $\left(0, \xi_{2}\right)$ and stays in $\mathscr{2}_{2}$, since it does not meet $-\mathscr{T}_{r}$. Hence $S_{w}<\infty$, and $w$ is of type (2).

There exists also a unique trajectory $\mathscr{T}_{3}$ converging to $(0,0)$ at $\infty$, ending up in $2_{1}$, since $(0,0)$ is a saddle point. It stays in the domain of $\mathscr{2}_{1}$ delimited by $\mathscr{T}_{1}, \mathscr{T}_{2}$, because $\mathscr{2}_{1}$ is backward invariant. Thus $\mathscr{T}_{3}$ converges to $M_{\ell}$ at $-\infty$, tangentially to $u_{1}$. And $y$ is increasing on $\mathbb{R}$ : indeed $y^{\prime}<0$ near $\pm \infty$, and $y$ cannot have two extremal points. Then $w$ is of type (3).

For any point $P=(\varphi, 0)$ with $\varphi>\varphi_{1}$, the trajectory $\mathscr{T}_{[P]}$ goes from $\mathscr{Q}_{1}$ into $2_{4}$, by Remark 2.1(i). It does not meet $\mathscr{T}_{r}$ or $\mathscr{T}_{1}$; hence it stays in $\mathscr{2}_{4}$ after $P$, and

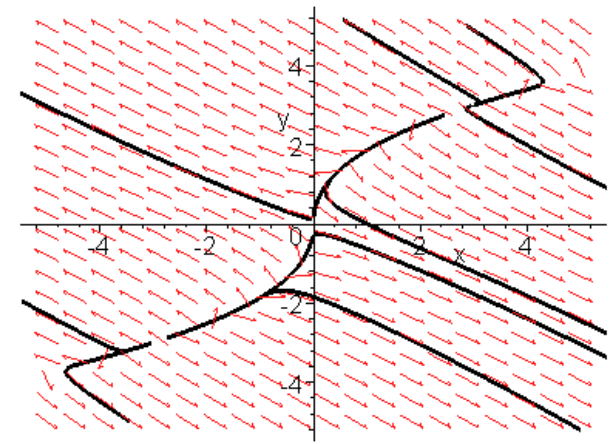

Figure 13. Theorem 6.4: $\varepsilon=-1,0<\alpha=2<\delta=3<N=4$. 
$S_{w}<\infty$. Before $P$, it stays in $\mathscr{2}_{1}$ because it does not meet $\mathscr{T}_{1}$ or $\mathscr{T}_{2}$, by the same remark. By Proposition 2.8, either $\lim _{\tau \rightarrow-\infty} \zeta=\alpha<\delta$, so $y^{\prime}=y(\delta-\zeta)>0$ near $-\infty$, and $\lim _{\tau \rightarrow-\infty} y=\infty$, which is impossible; or (necessarily) $\mathscr{T}_{[P]}$ converges to $M_{\ell}$, tangentially to $u_{1}$, and $\mathscr{T}_{[P]}$ is of type (2). Similarly, for any $P^{\prime}=(0, \xi)$ with $\xi>\xi_{2}$, the trajectory $\mathscr{T}_{\left[P^{\prime}\right]}$ goes from $2_{1}$ into $2_{2}$; it remains there after $P$ (so $S_{w}<\infty$ ) and remains in $2_{1}$ before $P$, converging to $M_{\ell}$ at $-\infty$, tangentially to $-u_{1}$. Thus $w$ is still of type (2).

The sets

$$
\begin{aligned}
& \mathscr{A}=\left\{P \in \mathscr{Q}_{1}: \mathscr{T}_{[P]} \cap\{(\varphi, 0): \varphi>0\} \neq \varnothing\right\}, \\
& \mathscr{B}=\left\{P \in \mathscr{2}_{1}: \mathscr{T}_{[P]} \cap\{(0, \xi): \xi>0\} \neq \varnothing\right\},
\end{aligned}
$$

are open and nonempty, so $\mathscr{A} \cup \mathscr{B} \neq \mathscr{2}_{1}$. There is at least one trajectory $\mathscr{T}_{4}$ in $\mathscr{2}_{1}$ converging to $M_{\ell}$ at $-\infty$ and such that $\lim _{\tau \rightarrow \infty} \zeta=\alpha$; thus $w$ is of type (4).

For any point $P$ in the bounded domain $\mathscr{R}^{\prime}$ of $\mathscr{2}_{1}$ delimited by $\mathscr{T}_{2}$ and $\mathscr{T}_{3}$, the trajectory $\mathscr{T}_{[P]}$ is confined to $\mathscr{R}^{\prime}$ before $P$, and $y$ has no maximal point; thus $y$ is monotone, and $\mathscr{T}$ converges to $M_{\ell}$ at $-\infty$. It cannot stay in $\mathscr{2}_{1}$ since it cannot converge to $(0,0)$. Thus it goes from $2_{1}$ into $2_{2}$ and stays there, because it does not meet $-\mathscr{T}_{r}$. Thus $S_{w}<\infty$, and $w$ is again of type (2).

For any $P$ in the domain of $\mathscr{2}_{1}$ delimited by $\mathscr{T}_{1}$ and $\mathscr{T}_{3}$, the trajectory $\mathscr{T}_{[P]}$ converges to $M_{\ell}$ at $-\infty$, tangentially to $u_{1}$; it enters $2_{4}$ and stays there. Thus $S_{w}<\infty$ and $w$ is of type (1). No trajectory can stay in $\mathscr{2}_{4}\left(\mathscr{2}_{2}\right)$ except $\mathscr{T}_{r}\left(-\mathscr{T}_{r}\right)$; thus all the solutions have been described, up to a symmetry.

Now we come to the case $\alpha<0$, and discuss according to the sign of $\alpha-p^{\prime}$. This situation is different from the case $\varepsilon=1, \delta<\min (\alpha, N)$ discussed on page 246, by Remark (i) on page 249 and part (i) of the next remark.

Remark 6.5. Assume $\varepsilon=-1$ and $\alpha<0$.

(i) The regular trajectory $\mathscr{T}_{r}$ starts in $\mathscr{2}_{1}$. There exists a unique trajectory $\mathscr{T}_{s}$ converging to $(0,0)$, lying in $2_{1}$ for large $\tau$, having an infinite slope at $(0,0)$, and satisfying $\lim _{r \rightarrow 0} r^{\eta} w=c>0$. If $\mathscr{T}_{s}$ does not stay in $\mathscr{2}_{1}$, then $\mathscr{T}_{r}$ does stay in it, and it is bounded and contained in the domain delimited by $\mathscr{2}_{1} \cap \mathscr{T}_{s}$, by Remark 2.1(i). If $\mathscr{T}_{r}$ is homoclinic, it stays in $\mathscr{2}_{1}$.

Conversely, if $\mathscr{T}_{s}$ stays in $\mathscr{2}_{1}$ and is not homoclinic, $\mathscr{T}_{r}$ does not stay in $\mathscr{2}_{1}$, for the following reason. $\mathscr{T}_{s}$ either converges to $M_{\ell}$ at $-\infty$ or has a limit cycle around it; if $\mathscr{T}_{r}$ stays in $\mathscr{2}_{1}$, either the corresponding $y$ is increasing, so $\lim _{\tau \rightarrow \ln } S_{w} Y / y=-1$; or $\lim _{\tau \rightarrow \infty} \zeta=\alpha<0$, by Propositions 2.15 and 2.8, so $\mathscr{T}_{r}$ enters $2_{4}$ and we reach a contradiction; or $y$ oscillates around $\ell$ near $\infty$, by Proposition 2.7, so it meets $\mathscr{T}_{s}$, which is impossible.

(ii) Any trajectory $\mathscr{T}$ is bounded near $-\infty$ from Propositions 2.8 and 2.10. Any trajectory $\mathcal{T}$ bounded at $\pm \infty$ converges to $(0,0)$ or $\pm M_{\ell}$, or its limit set $\Gamma_{ \pm}$at $\pm \infty$ is a limit cycle; or $\mathscr{T}_{r}$ is homoclinic and $\Gamma_{ \pm}=\overline{\mathscr{T}}_{r}$. 
(iii) If there exists a limit cycle around $(0,0)$, it also surrounds $\pm M_{\ell}$, by $(2-42)$ and (2-43).

Next we study the case $-p^{\prime} \leq \alpha$, where there is no cycle and no homoclinic orbit in $2_{1}$, by Theorem 2.20 .

Theorem 6.6. (i) Assume $\varepsilon=-1$ and $-p^{\prime}<\alpha<0<\delta<N$. Then all regular solutions have precisely one zero, and $S_{w}<\infty$. The function $w \equiv \ell r^{-\delta}$ is a solution. There exist solutions satisfying any one of these characterizations:

(1) $w$ is positive, $\lim _{r \rightarrow 0} r^{\delta} w=\ell$ and $\lim _{r \rightarrow \infty} r^{\eta} w=c>0$;

(2) $w$ has one zero, $\lim _{r \rightarrow 0} r^{\delta} w=\ell$, and $\lim _{r \rightarrow \alpha} r^{\alpha} w=L<0$;

(3) $w$ has one zero, $\lim _{r \rightarrow 0} r^{\delta} w=\ell$, and $S_{w}<\infty$;

(4) $w$ has two zeros, $\lim _{r \rightarrow 0} r^{\delta} w=\ell$, and $S_{w}<\infty$.

(ii) Assume $\alpha=-p^{\prime}$. Then the regular solutions, given by (1-8), have one zero, and $\lim _{r \rightarrow \alpha} r^{\alpha} w=L<0$. There exist solutions of type (1) and (4).

Up to symmetry, all solutions are as above.

Proof. (i) Assume $-p^{\prime}<\alpha<0$ (Figure 14, left). By Proposition 2.5, any solution $y$ has at most two zeros, and $Y$ has at most one zero.

First consider $\mathscr{T}_{s}$. The function $Y_{\alpha}$ defined by (2-3) with $d=\alpha$ satisfies $Y_{\alpha}=$ $O\left(e^{(\alpha-\eta) \tau}\right)$ near $\infty$, thus $\lim _{\tau \rightarrow \infty} Y_{\alpha}=0$. Then from Remark 2.6, $Y_{\alpha}$ is decreasing, thus $Y_{\alpha}>0$, and $\mathscr{T}_{s}$ stays in $\mathscr{2}_{1} \cup 2_{2}$. In fact it stays in $\mathscr{2}_{1}$, by Remark 2.1(i). From Propositions 2.8, 2.7, 2.11, and Theorem 2.20, $\mathscr{T}_{s}$ converges to $M_{\ell}$ at $-\infty$. Indeed if $\lim y=\infty$, then $\lim _{\tau \rightarrow \infty} \zeta=\alpha<0$; if $S_{w}<\infty$, then $\lim Y / y=-1$; which contradicts $Y>0$. Then $w$ is of type (1).

The trajectory $\mathscr{T}_{r}$ stays in $\mathscr{2}_{1} \cup \mathscr{2}_{2}$, and $y$ has precisely one zero, and $S_{w}<\infty$, so $\lim _{\tau \rightarrow \ln S_{w}} Y / y=-1$. We claim that $\mathscr{T}_{r}$ cannot stay in $\mathscr{2}_{1}$. Indeed, it cannot
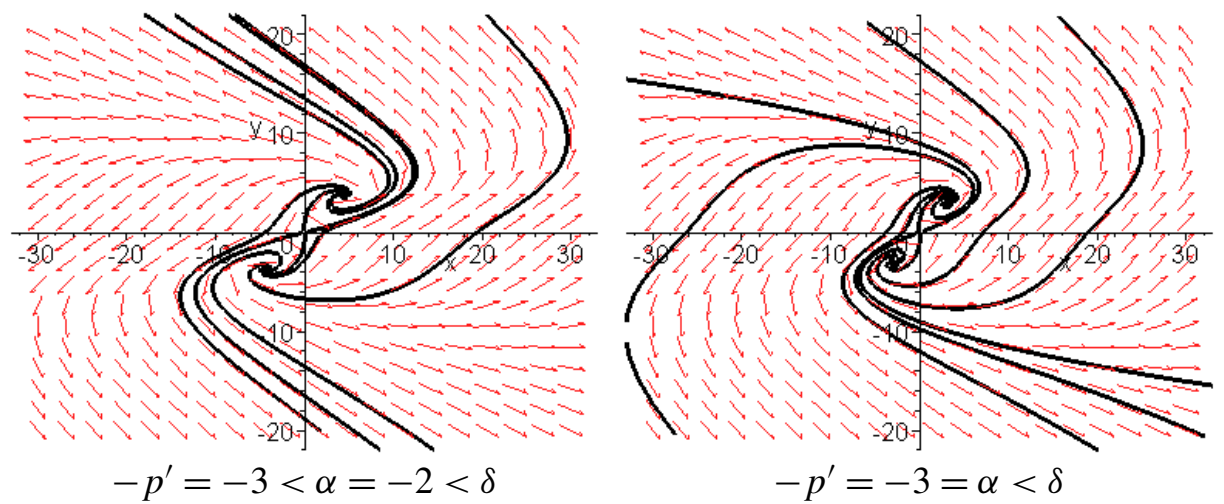

Figure 14. Theorem 6.6: $\varepsilon=-1, \delta=3<N / 2<N=9$. 
converge to $M_{\ell}$, which is a source, or oscillate around $\mathscr{2}_{1}$, because it does not meet $\mathscr{T}_{s}$, or tend to $\infty$, or satisfy $S_{w}<\infty$ with $Y>0$. Thus $y$ has precisely one zero, $\mathscr{T}_{r}$ enters $\mathscr{2}_{2}$ and stays in it. Moreover the corresponding $Y_{\alpha}$ satisfies $Y_{\alpha}^{\prime}>0$, or equivalently (6-1). Consider again the curve $\mathcal{N}_{\alpha}$ defined in (6-2). Here $\mathscr{T}_{r}$ stays strictly to the right of $\mathcal{N}_{\alpha}$, and $\mathscr{T}_{s}$ to the left of $\mathcal{N}_{\alpha}$.

For any $\bar{P}=(\varphi, 0)$ with $\varphi<0$, the trajectory $\mathscr{T}_{[\bar{P}]}$ enters $2_{3}$ after $\bar{P}$, by Remark 2.1(i). The solution going through $\bar{P}$ at $\tau=0$ satisfies $Y_{\alpha}(0)=0$; thus $Y_{\alpha}$ stays positive as before, and $Y_{\alpha}^{\prime}<0$, since $Y_{\alpha}$ has no maximal point, by Remark 2.6. Thus $\mathscr{T}_{[\bar{P}]}$ stays in $\mathscr{2}_{1} \cup \mathscr{2}_{2}$ before $\bar{P}$, to the left of $\mathcal{N}_{\alpha}$. It cannot stay in $\mathscr{2}_{2}$, by Propositions 2.7 and 2.8. As $\tau$ decreases, it enters $2_{1}$, and converges to $M_{\ell}$, by Theorem 2.20. If $S_{w}=\infty$, then $\lim |y|=\infty$ and $\lim _{\tau \rightarrow \infty} \zeta=\alpha<0$; this is impossible, since $\mathscr{T}_{[\bar{P}]}$ does not meet $-\mathscr{T}_{r}$. Thus $S_{w}<\infty, \lim Y / y=-1, \mathscr{T}_{[\bar{P}]}$ goes from $2_{3}$ into $2_{4}$ and stays in it, and $w$ is of type (4). The solution $y$ has precisely two zeros.

Next consider $\mathscr{T}_{[P]}$ for any $P=(\varphi, \xi) \in \mathcal{N}_{\alpha}$ with $\varphi<0$. The solution passing through $P$ at $\tau=0$ satisfies $Y_{\alpha}^{\prime}(0)=0$ and $Y_{\alpha}(0)>0$, and 0 is a minimal point. Therefore $Y_{\alpha}^{\prime \prime}(0)>0$; indeed, if $Y_{\alpha}^{\prime \prime}(\tau)=0$, we conclude from uniqueness that $Y_{\alpha}$ is constant on $\mathbb{R}$; then (2-6) yields $Y_{\alpha} \equiv 0$, since $\alpha \neq-p^{\prime}$. But this cannot be. Therefore $Y_{\alpha}^{\prime}(\tau)>0$ for $\tau>0, Y_{\alpha}^{\prime}(\tau)<0$ for $\tau<0$, and $\mathscr{T}_{[P]}$ stays in $\mathscr{2}_{1} \cup \mathscr{2}_{2}$, to the right of $\mathcal{N}_{\alpha}$ after $P$, with $y<0$ by Remark 2.1(i), and to the left of $\mathcal{N}_{\alpha}$ before $P$. As above it cannot stay in $2_{2}$ near $-\infty$, and converges to $M_{\ell}$. Suppose that it satisfies $S_{w}=\infty$. Then $\lim |y|=\infty, \lim _{\tau \rightarrow \infty} \zeta=\alpha$, and $\lim _{\tau \rightarrow \infty} y_{\alpha}=L<0$ by Proposition 2.9; hence $\lim _{\tau \rightarrow \infty} Y_{\alpha}=(\alpha L)^{p-1}$. As in Proposition 2.5(iii), we find $Y_{\alpha}^{\prime \prime}(\tau)>0$ for any $\tau>0$, which is impossible. Then $S_{w}<\infty$, so $\lim _{\tau \rightarrow \ln } S_{w} Y / y=-1$ and $w$ is of type (3).

Finally consider the domain $\mathscr{R}$ of $\mathscr{2}_{1} \cup \mathscr{Q}_{2}$ delimited by $\mathscr{T}_{r}$ and $\mathscr{T}_{s}$ and containing $\mathcal{N}_{\alpha}$. Form the sets

$$
\begin{aligned}
& \mathscr{A}=\left\{P \in \mathscr{R}: \mathscr{T}_{[P]} \cap \mathcal{N}_{\alpha} \neq \varnothing\right\}, \\
& \mathscr{B}=\left\{P \in \mathscr{R}: \mathscr{T}_{[P]} \cap\{(\xi, 0): \xi>0\} \neq \varnothing\right\},
\end{aligned}
$$

corresponding to trajectories of type (3) or (4). They are nonempty and open, since here again the intersection with $\mathcal{N}_{\alpha}$ is transverse $\left(\alpha \neq-p^{\prime}\right)$. Thus $\mathscr{A} \cup \mathscr{B}$ is distinct from $\mathscr{R}$ : there exists a trajectory in $\mathscr{R}$ that does not meet $\mathcal{N}_{\alpha}$; it converges to $M_{\ell}$ at $-\infty$ or oscillates around it, and it is located below $\mathcal{N}_{\alpha}$ in $\mathscr{2}_{2}$. It cannot satisfy $\lim _{\tau \rightarrow \ln S_{w}} Y / y=-1$, so $S_{w}=\infty$ and we have $\lim _{\tau \rightarrow \infty} \zeta=\alpha$. Hence $w$ is of type (2).

(ii) Assume $\alpha=-p^{\prime}$ (Figure 14, right). Then regular solutions have a different behavior: they are given explicitly by (1-8). They satisfy $Y_{-p^{\prime}} \equiv C$, thus $Y_{-p^{\prime}}^{\prime} \equiv 0$, 
thus $\mathscr{T}_{r}=\mathcal{M}_{-p^{\prime}}$. Here $y$ has a zero, and $S_{w}=\infty$, and $\lim _{\tau \rightarrow \infty} \zeta=-p^{\prime}$. As above $\mathscr{T}_{s}$ stays in $\mathscr{2}_{1}$ and $w$ is of type (1).

Next consider again $\mathscr{T}_{[\bar{P}]}$. The solution going through $\bar{P}$ at $\tau=0$ satisfies $Y_{-p^{\prime}}(0)=0$, thus $Y_{-p^{\prime}}$ stays negative for $\tau>0$ and $Y_{-p^{\prime}}^{\prime}<0$. Suppose that $S_{w}=\infty$, and $\lim _{\tau \rightarrow \infty} \zeta=-p^{\prime}$, then $\lim _{\tau \rightarrow \infty} y_{\alpha}=L>0, \lim _{\tau \rightarrow \infty} Y_{\alpha}=-(|\alpha| L)^{p-1}$. But as in (2-46), $Y_{\alpha}^{\prime \prime}(\tau)<0$ for any $\tau>0$, which leads to a contradiction. Then $S_{w}<\infty$ and $w$ is of type (4).

Finally suppose that there exists a trajectory $\mathscr{T} \neq \mathscr{T}_{r}$ staying in $\mathscr{2}_{1} \cup \mathscr{2}_{2}$. Then it converges to $M_{\ell}$, thus $Y_{\alpha}>0, S_{w}=\infty$, and $\lim _{\tau \rightarrow-\infty} Y_{\alpha}=\infty, \lim _{\tau \rightarrow \infty} Y_{\alpha}=C>0$. If $\mathcal{T}$ has a minimal point, then it has an inflection point where $Y_{\alpha}^{\prime}>0$, which as above is impossible. Then $Y_{\alpha}^{\prime}<0$; (2-6) yields

$$
(p-1) Y_{-p^{\prime}}^{\prime \prime}=Y_{-p^{\prime}}^{\prime}\left(e^{p^{\prime} \tau} Y_{-p^{\prime}}^{(2-p) /(p-1)}-N(p-1)\right)=Y_{-p^{\prime}}^{\prime}(Y-N(p-1)),
$$

and $\lim _{\tau \rightarrow \infty} Y=\infty$, so $Y_{-p^{\prime}}^{\prime \prime}<0$ for large $\tau$, which is impossible. Thus there exist no solutions of type (2) or (3).

We now come to the most difficult case: $\alpha<-p^{\prime}$.

Lemma 6.7. Assume $\varepsilon=-1$ and $\alpha<-p^{\prime}$. If $\delta<N / 2$ and $\alpha^{*}<\alpha$, either $\mathscr{T}_{r}$ has a limit cycle in $2_{1}$, or is homoclinic, or all regular solutions have at least two zeros. If $N / 2 \leq \delta<N$, they have at least two zeros.

Proof. In any case $M_{\ell}$ is a source. Suppose that $\mathscr{T}_{r}$ has no limit cycle in $\mathscr{2}_{1}$, or is not homoclinic (in particular it happens when $N / 2 \leq \delta<N$, by Proposition 2.11), and stays in $2_{1} \cup 2_{2}$, thus $Y$ stays positive. Then from Propositions 2.8, 2.9 and 2.15, either $\lim _{\tau \rightarrow-\infty} y=\infty, \lim _{\tau \rightarrow \infty} y_{\alpha}=L \neq 0, \lim _{\tau \rightarrow \infty} Y_{\alpha}=(\alpha L)^{p-1}$, or $S_{w}<\infty$. In any case, for any $d \in\left(\alpha,-p^{\prime}\right)$, the function $Y_{d}=e^{(d-\alpha) \tau} Y_{\alpha}$ satisfies $\lim _{\tau \rightarrow \ln S_{w}} Y_{d}=\infty=\lim _{\tau \rightarrow \infty} Y_{d}$. Then it has a minimum point, and this contradicts (2-15). Thus $\mathscr{T}_{r}$ enters $2_{3}$. If it stays in it, it has a limit cycle; then $-\mathscr{T}_{r}$ has a limit cycle in $\mathscr{2}_{1}$. But $-\mathscr{T}_{r}$ does not meet $\mathscr{T}_{r}$, and $M_{\ell}$ is in the domain of $\mathscr{2}_{1}$ delimited by $\mathscr{T}_{r}$, since $\mathscr{T}_{r}$ meets $\mathcal{M}$ to the right of $M_{\ell}$, by (2-16); this is impossible. Then $\mathscr{T}_{r}$ enters $2_{4}$, and $y$ has at least two zeros.

Theorem 6.8. Assume $\varepsilon=-1$ and $\delta<N / 2, \alpha<-p^{\prime}$. Then $w(r)=\ell r^{-\delta}$ is still a solution.

(i) There exists a (minimal) critical value $\alpha^{\text {crit }}$ of $\alpha$, such that

$$
\alpha^{*}<\alpha^{\text {crit }}<\min \left(-p^{\prime}, \alpha_{2}\right)<0,
$$

and $\mathscr{T}_{r}$ is homoclinic: all regular solutions have constant sign and satisfy

$$
\lim _{r \rightarrow \infty} r^{\eta} w=c \neq 0
$$



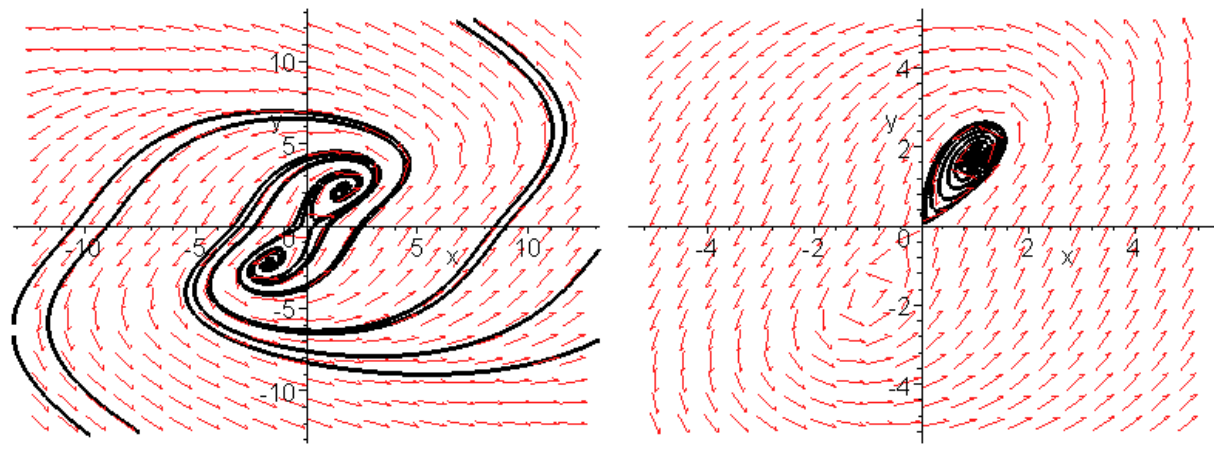

$$
\alpha=-5<-p^{\prime}=-3<0<\delta
$$

$$
\alpha=-7.4<0<\delta
$$
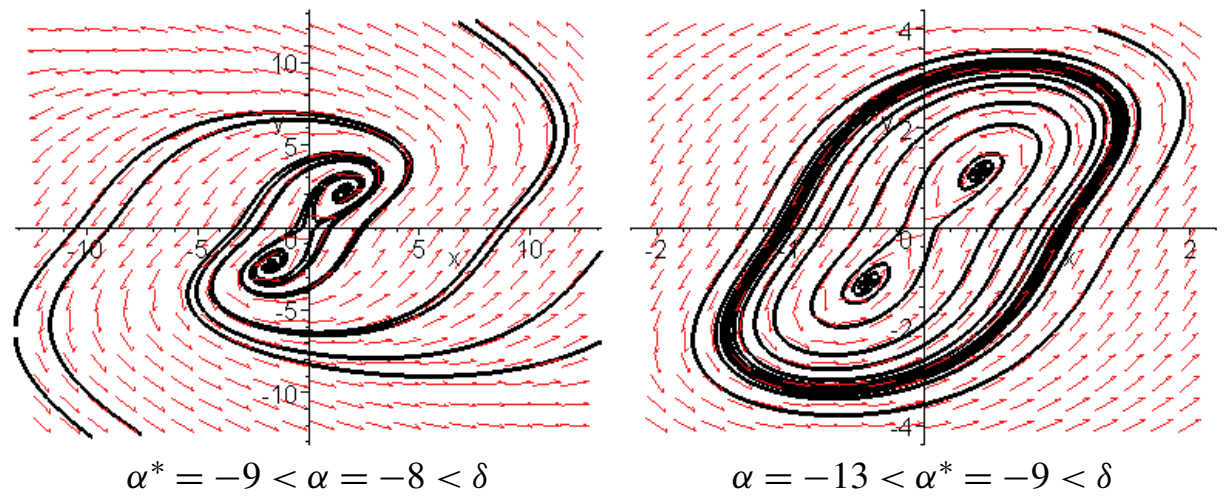

$$
\alpha=-13<\alpha^{*}=-9<\delta
$$

Figure 15. Theorem 6.8: $\varepsilon=-1, \delta=3<N / 2<N=9$.

(ii) For any $\alpha \in\left(\alpha^{*}, \alpha^{\text {crit }}\right)$ there does exist a cycle in $2_{1}$; equivalently there exist solutions such that $r^{\delta} w$ is periodic in $\ln r$. All regular solutions have constant sign and $r^{\delta} w$ is asymptotically periodic in $\ln r$. There exist positive solutions such that $\lim _{r \rightarrow 0} r^{\delta} w=\ell$ and $r^{\delta} w$ is asymptotically periodic in $\ln r$.

(iii) For any $\alpha \leq \alpha^{*}$, there does not exist such a cycle, regular solutions have constant sign, and $\lim _{r \rightarrow \infty} r^{\delta}|w|=\ell$.

(iv) For any $\alpha<\alpha^{\text {crit }}$, there exists also a cycle surrounding $(0,0)$ and $\pm M_{\ell}$, thus $w$ is changing sign and $r^{\delta} w$ is periodic in $\ln r$. There exist solutions oscillating near 0 , and $r^{\delta} w$ is asymptotically periodic in $\ln r$, and $\lim _{r \rightarrow \infty} r^{\eta} w=c \neq 0$. There exist solutions oscillating near 0 , and $r^{\delta} w$ is asymptotically periodic in $\ln r$, and $S_{w}<\infty$ or $\lim _{r \rightarrow \infty} r^{\alpha} w=L \neq 0$.

Proof. (i) For any $\alpha \in\left(\alpha_{1}, \alpha_{2}\right)$, such that $\alpha \leq-p^{\prime}$ we have three possibilities, by Remark 6.5:

- $\mathscr{T}_{s}$ converges to $M_{\ell}$ at $-\infty$, spiraling around this point, since $\alpha$ is a spiral point, or it has a limit cycle around $M_{\ell}$. Then $\mathscr{T}_{s}$ meets the set $\mathscr{E}=\{(\ell, Y)$ : 
$\left.Y>(\delta \ell)^{p-1}\right\}$ at a first point $\left(\ell, Y_{s}(\alpha)\right)$; and $\mathscr{T}_{r}$ meets $\mathscr{E}$ at a last point $\left(\ell, Y_{r}(\alpha)\right)$ such that $Y_{r}(\alpha)-Y_{s}(\alpha)>0$. Moreover $\mathscr{T}_{r}$ enters $2_{2}$, by Proposition 2.8. See Figure 15, top left.

- $\mathscr{T}_{s}$ enters $2_{4}$; hence $\mathscr{T}_{r}$ converges to $M_{\ell}$ at $\infty$ and spirals around this point, or it has a limit cycle around $M_{\ell}$. Then $\mathscr{T}_{s}$ meets $\mathscr{E}$ at a last point $\left(\ell, Y_{s}(\alpha)\right)$, $\mathscr{T}_{r}$ meets $\mathscr{E}$ at a first point $\left(\ell, Y_{r}(\alpha)\right)$ such that $Y_{r}(\alpha)-Y_{s}(\alpha)<0$. See Figure 15 , bottom row.

- $\mathscr{T}_{r}$ is homoclinic, or equivalently $Y_{r}(\alpha)-Y_{S}(\alpha)=0$. See Figure 15, top right.

Now the function $\alpha \mapsto h(\alpha)=Y_{r}(\alpha)-Y_{s}(\alpha)$ is continuous. If $-p^{\prime}<\alpha_{2}$, then $h\left(-p^{\prime}\right)$ is defined and $h\left(-p^{\prime}\right)>0$, by Theorem 6.6. If $\alpha_{2} \leq-p^{\prime}$, we observe that for $\alpha=\alpha_{2}$, by Theorem 2.18, $\mathscr{T}_{r}$ must leave $\mathscr{2}_{1}$ (because $\alpha_{2}$ is a source) and does so transversally; thus the same holds for $\alpha=\alpha_{2}-\gamma$ if $\gamma>0$ is small enough. Therefore $\mathscr{T}_{s}$ stays in $\mathscr{2}_{1}$ by Remark 6.5, so $h\left(\alpha_{2}-\gamma\right)>0$. If $\alpha \leq \alpha^{*}$, then $M_{\ell}$ is a sink or a weak sink, by Theorem 2.16; therefore $\mathscr{T}_{s}$ cannot converge to $M_{\ell}$ at $-\infty$. By Theorem 2.19, there are no cycles in $2_{1}$ and no homoclinic orbits. By Remark 6.5, $\mathscr{T}_{s}$ cannot stay in $\mathscr{2}_{1}$; hence $\mathscr{T}_{r}$ stays in $\mathscr{2}_{1}$ and is bounded and converges at $\infty$ to $M_{\ell}$. Thus $h(\alpha)<0$ for $\alpha_{1}<\alpha \leq \alpha^{*}$, so there exists at least an $\alpha^{\text {crit }} \in\left(\alpha^{*}, \min \left(-p^{\prime}, \alpha_{2}\right)\right.$ such that $h\left(\alpha^{\text {crit }}\right)=0$. If it is not unique, we choose the smallest one.

(ii) Let $\alpha>\alpha^{*}$. The existence and uniqueness of such a cycle in $2_{1}$ follows from Theorem 2.16 if $\alpha-\alpha^{*}$ is small enough (Figure 15, lower left). For any $\alpha \in$ $\left(\alpha^{*}, \alpha^{\text {crit }}\right)$, we still have existence: indeed, $h(\alpha)<0$ on this interval, so $\mathscr{T}_{r}$ stays in $\mathscr{2}_{1}$, and $\mathscr{T}_{r}$ cannot converge to $M_{\ell}$ at $\infty$, hence it has a limit cycle around $M_{\ell}$ at $\infty$. Since $M_{\ell}$ is a source, there also exist trajectories converging to $M_{\ell}$ at $-\infty$, with a limit cycle at $\infty$. And $\mathscr{T}_{s}$ does not stay in $\mathscr{2}_{1}$, and it is bounded at $-\infty$. Thus it has a limit cycle at $-\infty$ surrounding $(0,0)$ and $\pm M_{\ell}$.

(iii) Let $\alpha \leq \alpha^{*}$ (Figure 15, lower right). Then $\mathscr{T}_{r}$ stays in $\mathscr{2}_{1}$, is bounded on $\mathbb{R}$, and converges to $M_{\ell}$ at $\infty$, while $\mathscr{T}_{s}$ does not stay in $\mathscr{2}_{1}$ as above. Thus $\mathscr{T}_{s}$ has a limit cycle at $-\infty$, containing the three stationary points.

(iv) For any $\alpha<\alpha^{\text {crit }}$ apart from $\mathscr{T}_{r}$ and the cycles, all trajectories have a limit cycle at $-\infty$ containing the three stationary points. By Theorem 2.21, all the cycles are contained in a ball $B$ of $\mathbb{R}^{2}$. Take any point $P$ exterior to $B$. By Remark 6.5, $\mathscr{T}_{[P]}$ has a limit cycle at $-\infty$ contained in $B$ and cannot have a limit cycle at $\infty$. Therefore $y$ has constant sign near $\ln S_{w}$. By Proposition 2.8, either $S_{w}<\infty$ or $y$ is defined near $\infty$ and $\lim _{\tau \rightarrow \infty} \zeta=L, \lim _{r \rightarrow \infty} r^{\alpha} w=L$.

Finally we consider the case $N / 2 \leq \delta$, where no cycle can exist. 
Theorem 6.9. Assume $\varepsilon=-1$ and $\alpha<0<N / 2 \leq \delta<N$. Then all solutions of $\left(E_{w}\right)$ have a finite number of zeros, and $w(r)=\ell r^{-\delta}$ is a solution. If $-p^{\prime} \leq \alpha$, Theorem 6.6 applies. If $\alpha<-p^{\prime}$, there exist positive solutions such that $\lim _{r \rightarrow 0} r^{\delta} w=\ell$ and $\lim _{r \rightarrow \infty} r^{\eta} w=c>0$. All regular solutions have the same number $m \geq 2$ of zeros. All other solutions satisfy $\lim _{r \rightarrow-\infty} r^{\delta} w= \pm \ell$, and have $m$ or $m+1$ zeros; there exist solutions with $m+1$ zeros.

Proof. By Proposition 2.11, all solutions have a finite number of zeros, and any solution is monotone near 0 and $\ln S_{w}$, or converges to $\pm M_{\ell}$. By Remark 6.5, apart from $\mathscr{T}_{r}$, all trajectories converge to $\pm M_{\ell}$ at $-\infty$. The functions $V$ and $W$ are nonincreasing. The trajectory $\mathscr{T}_{s}$ satisfies $\lim _{\tau \rightarrow \infty} V=\lim _{\tau \rightarrow \infty} W=0$, so $V \geq 0, W \geq 0$. If $y$ has a zero at some point $\tau$, then $W(\tau)=-|Y(\tau)|^{p^{\prime}} / p^{\prime}$, which is impossible. If $Y$ has a zero at some point $\theta$, then $V(\theta)=-Y^{\prime}(\theta)^{2} / 2$, also a contradiction. Thus $\mathscr{T}_{s}$ stays in $\mathscr{2}_{1}$. By Remark 6.5 and Proposition $2.11, \mathscr{T}_{r}$ does not stay in $\mathscr{2}_{1}$, but enters $\mathscr{2}_{2}$. By Lemma $6.7, \mathscr{T}_{r}$ enters $\mathscr{2}_{4}$, and $y$ has at least two zeros. Let $m$ be the number of its zeros. Then $\mathscr{T}_{r}$ cuts the axis $y=0$ at points $\left(0, \xi_{1}\right), \ldots,\left(0, \xi_{m}\right)$. Consider any trajectory $\mathscr{T}_{[P]}$ with $P=(0, \xi)$, where $\xi>\left|\xi_{i}\right|$ for $1 \leq i \leq m$. It cannot intersect $\mathscr{T}_{r}$ or $-\mathscr{T}_{r}$, so $y$ has $m+1$ zeros. Any trajectory has $m$ or $m+1$ zeros, because it does not meet $\mathscr{T}_{r}$ or $-\mathscr{T}_{r}$ or $\mathscr{T}_{[P]}$. And $S_{w}<\infty$ or $\lim _{r \rightarrow \infty} r^{\alpha} w=L \neq 0$.

\section{Acknowledgment}

We thank Hector Giacomini (University of Tours) for interesting discussions on the existence of cycles in dynamical systems.

\section{References}

[Anderson and Leighton 1968] L. R. Anderson and W. Leighton, "Liapunov functions for autonomous systems of second order", J. Math. Anal. Appl. 23 (1968), 645-664. MR 37 \#5488 Zbl 0186.41602

[Barenblatt 1952] G. I. Barenblatt, "On self-similar motions of a compressible fluid in a porous medium”, Akad. Nauk SSSR. Prikl. Mat. Meh. 16 (1952), 679-698. MR 14,699h Zbl 0047.19204

[Bidaut-Véron 1989] M.-F. Bidaut-Véron, "Local and global behavior of solutions of quasilinear equations of Emden-Fowler type", Arch. Rational Mech. Anal. 107:4 (1989), 293-324. MR 90f: $35066 \mathrm{Zbl} 0696.35022$

[Bidaut-Véron 2006a] M. F. Bidaut-Véron, “The $p$-Laplace heat equation with a source term: selfsimilar solutions revisited”, Adv. Nonlinear Stud. 6:1 (2006), 69-108. MR MR2196892

[Bidaut-Véron 2006b] M. F. Bidaut-Véron, "Self-similar solutions of the $p$-Laplace heat equation: the case $p>2$ ", 2006. In preparation.

[Chasseigne and Vazquez 2002] E. Chasseigne and J. L. Vazquez, "Theory of extended solutions for fast-diffusion equations in optimal classes of data. Radiation from singularities", Arch. Ration. Mech. Anal. 164:2 (2002), 133-187. MR 2003i:35148 Zbl 1018.35048 
[Chicone and Tian 1982] C. Chicone and J. H. Tian, "On general properties of quadratic systems", Amer. Math. Monthly 89:3 (1982), 167-178. MR 84f:34044 Zbl 0466.34011

[DiBenedetto and Herrero 1990] E. DiBenedetto and M. A. Herrero, "Nonnegative solutions of the evolution $p$-Laplacian equation. Initial traces and Cauchy problem when $1<p<2$ ", Arch. Rational Mech. Anal. 111:3 (1990), 225-290. MR 92g:35088

[Gil and Vázquez 1997] O. Gil and J. L. Vázquez, "Focusing solutions for the $p$-Laplacian evolution equation", Adv. Differential Equations 2:2 (1997), 183-202. MR 97g:35069 Zbl 1023.35514

[Hale and Koçak 1991] J. K. Hale and H. Koçak, Dynamics and bifurcations, Texts in Applied Mathematics 3, Springer, New York, 1991. MR 93e:58047 Zbl 0745.58002

[Hubbard and West 1995] J. H. Hubbard and B. H. West, Differential equations: a dynamical systems approach, v. 2: Higher dimensional systems, Texts in Applied Mathematics 18, Springer, New York, 1995. MR 96e:34001 Zbl 0824.34001

[Kamin and Vázquez 1992] S. Kamin and J. L. Vázquez, "Singular solutions of some nonlinear parabolic equations”, J. Anal. Math. 59 (1992), 51-74. MR 94e:35079 Zbl 0802.35066

[Qi and Wang 1999] Y.-W. Qi and M. Wang, "The global existence and finite time extinction of a quasilinear parabolic equation", Adv. Differential Equations 4:5 (1999), 731-753. MR 2000d:35106 Zbl 0959.35104

[Vazquez and Véron 1996] J. L. Vazquez and L. Véron, "Different kinds of singular solutions of nonlinear parabolic equations", pp. 240-249 in Nonlinear problems in applied mathematics: in honor of Ivar Stakgold on his 70th birthday, Soc. Ind. Appl. Math., Philadelphia, 1996. Zbl 0886.35078

[Zhao 1993] J. N. Zhao, "The asymptotic behaviour of solutions of a quasilinear degenerate parabolic equation”, J. Differential Equations 102:1 (1993), 33-52. MR 94c:35040 Zbl 0816.35070

Received February 4, 2005. Revised January 2, 2006.

MARIE Françoise Bidaut-VÉron

LABORATOIRE De MATHEMATIQUes ET Physique ThÉORIQUe

CNRS UMR 6083

FACUlté Des SCIENCES ET TEChNiQues

PARC GRANDMONT

37200 TOURS

FRANCE

veronmf@univ-tours.fr 